\title{
ANALYSIS OF AN INFLATABLE GOSSAMER DEVICE TO EFFICIENTLY DE-ORBIT CUBESATS
}

\author{
A Thesis \\ Presented to \\ the Faculty of California Polytechnic State University \\ San Luis Obispo
}

\author{
In Partial Fulfillment \\ of the Requirements for the Degree \\ Master of Science in Aerospace Engineering
}

by

Robert A. Hawkins, Jr.

December 2013 
(C) 2013

Robert A. Hawkins, Jr.

ALL RIGHTS RESERVED 
TITLE: Analysis of an Inflatable Gossamer Device to Efficiently De-Orbit CubeSats

AUTHOR: Robert A. Hawkins, Jr.

DATE SUBMITTED: $\quad$ December 2013

COMMITTEe CHAIR: Kira Abercromby, Ph.D.

Assistant Professor

Aerospace Engineering Department

COMMITTEE MEMBER: Eric Mehiel, Ph.D.

Associate Professor

Aerospace Engineering Department

COMMITTEE MEMBER: Kim Aaron, Ph.D.

Chief Engineer

Global Aerospace Corporation

COMMITTEE MEMBER: Dan Wait, M.S. Systems Engineer

Tyvak Nano-Satellite Systems 


\begin{abstract}
Analysis of an Inflatable Gossamer Device to Efficiently De-Orbit CubeSats
\end{abstract} Robert A. Hawkins, Jr.

There is an increased need for spacecraft to quickly and efficiently de-orbit themselves as the amount of debris in orbit around Earth grows. Defunct spacecraft pose a significant threat to the LEO environment due to their risk of fragmentation. If these spacecraft are de-orbited at the end of their useful life their risk to future spacecraft is greatly lessened. A proposed method of efficiently de-orbiting spacecraft is to use an inflatable thin-film envelope to increase the body's area to mass ratio and thusly shortening its orbital lifetime. The system and analysis presented in this project is sized for use on a CubeSat as they are an effective utility as a technology demonstration platform. Analysis has been performed to characterize the orbital dynamics of high area to mass ratio spacecraft as well as the leak rate of such an inflatable device in a vacuum environment. Results show that a $1 \mathrm{U}$ CubeSat can be de-orbited using a 1.7 meter diameter spherical device in just under one year while using 0.7 grams of inflating gas, this is compared to over 25 years without any method of post-mission disposal. 


\section{ACKNOWLEDGMENTS}

I would first like to thank Dr. Abercromby for all of her help and guidance throughout the years of classes, senior project work, and thesis work. Kim Aaron and Kerry Nock of Global Aerospace Corporation for all of their insight and time. A debt of gratitude is due to Dan Wait, Dr. Mehiel, and all my teachers of past years.

The number of friends and family which have been by my side throughout my time at Cal Poly are too many to name, all of you I owe thanks. Jason, you're my go-to guy for bouncing ideas off of, this last year would have been even more tough without you. Finally, a heartfelt thank you to my parents, without your never-ending support I would not be where I am now. 


\section{TABLE OF CONTENTS}

LIST OF TABLES viii

LIST OF FIGURES Ix - ix

1 Introduction 1

2 Orbital Dynamics 5

2.1 Non-uniform Gravity Field of Earth . . . . . . . . . . . . . . . 6

2.2 Atmospheric Drag . . . . . . . . . . . . . . . . . . . . . . . 9

2.2.1 Atmospheric Density . . . . . . . . . . . . 10

2.2.2 Coefficient of Drag . . . . . . . . . . . . . 13

2.3 Solar Radiation Pressure . . . . . . . . . . . . . . . . . 27

2.4 Earth's Magnetic Field . . . . . . . . . . . . . . . . . 28

2.5 N-Body Perturbations . . . . . . . . . . . . . . . 28

3 Current Work in Spacecraft Disposal 30

3.1 Natural Decay . . . . . . . . . . . . . . . . . . 31

3.2 Propulsion . . . . . . . . . . . . . . . . . . . . 32

3.3 Drag Tether . . . . . . . . . . . . . . . . 34

3.4 Electromagnetic Tether . . . . . . . . . . . . . . 36

3.5 2-D Sail . . . . . . . . . . . . . . . . . . 37

3.6 3-D Device. . . . . . . . . . . . . . . . . . . . . 39

4 Analysis $\quad 42$

4.1 Orbital Debris and Micrometeorite Flux . . . . . . . . . . . . . 42

4.1.1 Orbital Debris Flux . . . . . . . . . . . . . . . 44

4.1 .2 Micrometeorite Flux . . . . . . . . . . . . . . . . 46

4.2 Hypervelocity Impacts . . . . . . . . . . . . . . . . . . . . . . . . 48 
4.3 Rarefied Gas Flow Through an Orifice . . . . . . . . . . . . . . . 51

5 Results $\quad 52$

5.1 System Sizing and Results . . . . . . . . . . . . . . 55

5.2 Leak Analysis Results . . . . . . . . . . . . . . . 61

5.3 System Design . . . . . . . . . . . . . . . . 67

5.4 Comparison with a 2-D Sail . . . . . . . . . . . 71

5.4.1 Effectiveness..................... 72

6 Conclusion $\quad 75$

6.1 Future Work . . . . . . . . . . . . . . . . . 75

$\begin{array}{ll}\text { BIBLIOGRAPHY } & 77\end{array}$

$\begin{array}{ll}\text { APPENDICES } & 82\end{array}$

A Data Tables $\quad 82$

B DSMC Results $\quad 86$ 


\section{LIST OF TABLES}

3.1 End of life requirements for spacecraft in MEO . . . . . . . . . . . 30

4.1 Assumed densities of micrometeorites based on diameter . . . . . 47

5.1 De-orbit results using different orbital perturbations . . . . . . . . 53

5.2 Orbit propagator settings and parameters . . . . . . . . . 53

5.3 Membrane area densities . . . . . . . . . . . . . . 57

5.4 Hole size and particle flux parameters for orbital debris particles . 62

5.5 Hole size and particle flux parameters for micrometeorites particles 63

5.6 Miniaturized cool gas generator parameters . . . . . . . . . . 68

5.7 System design results . . . . . . . . . . . . . . . . . . 69

5.8 Nanosail D-2 de-orbit values . . . . . . . . . . . . . . . . 72

5.9 CP5 Measurements . . . . . . . . . . . . . . . . . . . 73

5.10 Effectiveness of an inflatable device versus other PMD systems . . 73

A.1 U.S. Standard Atmosphere 1976 . . . . . . . . . . . . . . . . . . . 82

A.2 Inclination dependent orbital debris function . . . . . . . . . 83

B.1 DSMC results for drag coefficient and dynamic pressure . . . . . 87 


\section{LIST OF FIGURES}

1.1 Historical orbital debris population by debris type . . . . . . . . 2

1.2 Prediction of the orbital debris population by altitude regime . . . 3

1.3 Analysis of the growth of the orbital debris environment . . . . . 4

2.1 Magnitude of several different orbital perturbations compared to the acceleration from Earth, versus orbital altitude . . . . . . . 7

2.2 A visual of the ECI and ECEF reference frames . . . . . . . . . . 8

2.3 A comparison of the U.S. Standard Atmosphere and the NRLMSISE00 model at solar maximum and solar minimum, for a range of altitudes . . . . . . . . . . . . . . . . . . 12

2.4 Contour of atmospheric density based on altitude and years since the last solar minimum . . . . . . . . . . . . . . . . . . . . . . . 13

2.5 Estimation of drag coefficient based on body shape and altitude . 14

2.6 A range of Knudsen numbers showing associated valid solvers . . 15

2.7 A visual representation of fully specular, diffuse, and quasi-specular refection . . . . . . . . . . . . . . . 16

2.8 A visualization of fully specular refection of a sphere and flat plate 17

2.9 Drag Coefficient for Diffuse, DRIA, and CLL modes, and a curve from Pardini's observation . . . . . . . . . . . . . . . 20

2.10 Flow pressure and velocity results from a DSMC simulation at 100 $\mathrm{km}$ altitude .................... . . . 25

2.11 Contour of coefficient of drag values based on time since solar minimum and altitude . . . . . . . . . . . . 26

2.12 Coefficient of drag values based on altitude, taken at three solar conditions .................... 26 26

3.1 Relationship between area to mass ratio, altitude, and de-orbit time 31 
3.2 NASA's reference to area to mass ratio to de-orbit within 25 years, given apogee and perigee . . . . . . . . . . . . . 32

3.3 Estimated delta-v and fuel to perform a reentry burn . . . . . . 33

3.4 Recent altitude history for Landsat $5 \ldots \ldots \ldots$

3.5 Concept of an electromagnetic tether system . . . . . . . . . 36

3.6 Electromagnetic tether orbital decay rates based on inclination . . 37

3.7 Simulated cross sectional area over time at $620 \mathrm{~km}$ and $450 \mathrm{~km}$ of a 2 -D body in orbit . . . . . . . . . . . . 38

3.8 Diagram showing the components of a structurally-supported 3-D device . . . . . . . . . . . . . . . . 40

3.9 Rendering of a spacecraft with an attached inflatable device . . . 41

4.1 Analysis flow chat . . . . . . . . . . . . . . . . 43

4.2 Orbital debris flux of objects $1 \mu \mathrm{m}$ to $1 \mathrm{~cm}$, given a sun-synchronous orbit in 2013, at various altitudes. . . . . . . . . . . . . . . 46

4.3 Micrometeorite flux of objects $1 \mu \mathrm{m}$ to $1 \mathrm{~cm}$, at various altitudes . 48

4.4 Three types of hypervelocity impacts: cratering, near incipient penetration, and complete penetration . . . . . . . . . . 49

4.5 Outcomes of hypervelocity impact on thin-film material . . . . . 50

4.6 Particle diameter and resulting holeing area $\ldots \ldots \ldots$. . . . 51

5.1 Comparison of STK and orbit propagator results from a simulation of an $800 \mathrm{~km}$ zero inclination orbit . . . . . . . . . . . 54

5.2 De-orbit time, in years, based on starting altitude and ballistic

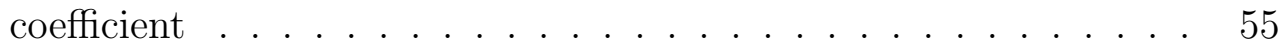

5.3 De-orbit time based on inflatable device size . . . . . . . . . . 56

5.4 Mass of membrane material based in total device diameter . . . 57

5.5 Stored volume of membrane material based in total device diameter 58

5.6 De-orbit time based on time since the last solar minimum using the NRLMSISE-00 atmospheric model . . . . . . . . . . . . 59

5.7 Reentry date based on deployment date using the NRLMSISE-00 atmospheric model . . . . . . . . . . . . . . . . . 60

5.8 Orbital lifetime over a range of altitudes, with three different atmospheric scenarios . . . . . . . . . . . . . . 60 
5.9 System failure altitude and its the resulting orbital lifetime and cumulative inflation gas used . . . . . . . . . . . . . . . . . 61

5.10 Hole area over time for a $1.7 \mathrm{~m}$ diameter device starting at an 800 $\mathrm{km}$ sun-synchronous orbit . . . . . . . . . . . . 63

5.11 Minimum internal to external pressure ratio over time . . . . . 65

5.12 Cumulative gas used over time for a $1.7 \mathrm{~m}$ diameter device starting at an $800 \mathrm{~km}$ sun-synchronous orbit in 2013 . . . . . . . . . 66

5.13 Gas requirements over a range of altitudes, with three different atmospheric scenario . . . . . . . . . . . . . . 66

5.14 Gas requirements based on deployment date . . . . . . . . . 67

5.15 Miniaturized cool gas generator by CCG Technologies . . . . . . 68

5.16 System with top released $\ldots \ldots \ldots$. . . . . . . . . . 70

5.17 System seen from the bottom _ . . . . . . . . . . 70

5.18 System in stored configuration . . . . . . . . . . . . . 71

B.1 Flow pressure and velocity results from a DSMC simulation at 500

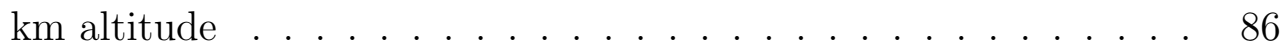




\section{NOMENCLATURE}

\section{English Characters}

\begin{tabular}{|c|c|}
\hline A & Area \\
\hline B & Magnetic Field \\
\hline $\mathrm{BC}$ & Ballistic Coefficient \\
\hline $\mathrm{C}$ & Coefficient \\
\hline $\mathrm{E}$ & Energy \\
\hline $\mathrm{E}$ & Young's Modulus \\
\hline $\mathrm{F}$ & Force \\
\hline G & Gravitational Constant \\
\hline $\mathrm{H}$ & Scale Height \\
\hline $\mathrm{J}$ & Zonal Harmonic \\
\hline K & Langmuir Fitting Parameter \\
\hline $\mathrm{K}_{N}$ & Knudsen Number \\
\hline $\mathrm{L}$ & Characteristic Length \\
\hline $\mathrm{P}$ & Pressure \\
\hline $\mathrm{P}$ & Penetration Thickness \\
\hline $\mathrm{P}_{O}$ & Partial Pressure of Atomic Oxygen \\
\hline $\mathrm{R}$ & Aspherical Potential \\
\hline S & 13 Month Smoothed F10.7 Solar Flux \\
\hline $\mathrm{T}$ & Temperature \\
\hline $\mathrm{U}$ & Energy \\
\hline $\mathrm{V}$ & Velocity \\
\hline $\mathrm{a}$ & Acceleration \\
\hline $\mathrm{d}$ & Particle Diameter \\
\hline $\mathrm{h}$ & Altitude \\
\hline $\mathrm{k}_{B}$ & Boltzmann's Constant \\
\hline $\mathrm{m}$ & Mass \\
\hline $\mathrm{m}$ & Molecular Mass \\
\hline $\bar{m}$ & Free-stream Molecular Mass \\
\hline$\dot{m}$ & Mass Flow Rate \\
\hline $\mathrm{n}$ & Number Density \\
\hline $\mathrm{p}$ & Annual Growth Rate of Mass in Orbit \\
\hline q & Charge \\
\hline q & Annual Growth Rate of Orbital Debris \\
\hline q' & Annual Growth Rate of Orbital Debris \\
\hline $\mathrm{r}$ & Hole Radius \\
\hline $\mathrm{s}$ & Speed Ratio \\
\hline $\mathrm{t}$ & Year \\
\hline $\mathrm{v}$ & Impact Speed \\
\hline $\mathrm{v}_{m p}$ & Most Probable Velocity \\
\hline
\end{tabular}


Other Characters

$\begin{array}{ll}\theta^{\prime} & \text { Portion of Surface Covered by Adsorbate } \\ \alpha & \text { Energy Accommodation Coefficient } \\ \alpha_{s} & \text { Adsorbate Energy Accommodation Coefficient } \\ \lambda & \text { Mean Free Path } \\ \mu & \text { Standard Gravitational Parameter } \\ \mu & \text { Ratio of Free-stream to Surface Molecular Mass } \\ \rho & \text { Density } \\ \sigma & \text { Momentum Accommodation Coefficient } \\ \phi & \text { Latitude } \\ \omega & \text { Rotational Velocity }\end{array}$

Subscripts

2

$\mathrm{D}$

I I Direction

J J Direction

K K Direction

$\mathrm{O} \quad$ Atomic Oxygen

R Reflectivity

SR Solar Radiation

i Incident

k Kinetic

l Local

n Normal

o Reference

p Particle

r Reflected

s Surface

sat Satellite

$\mathrm{t}$ Tangential

$\oplus \quad$ Earth

$\odot$ Sun

$\infty \quad$ Free-stream 


\section{Introduction}

A spacecraft orbiting Earth is under the constant threat of collision from other orbiting bodies. Functional spacecraft often have well defined trajectories and may even have the ability to perform evasive maneuvers to avoid spacecraftto-spacecraft collisions, the majority of the risk of collisions is due to orbital debris. Simply, orbital debris is anything that is in space which does not serve a useful purpose; rocket bodies, defunct spacecraft, mission-related debris, accidental explosion fragments, and collision remains are all classified as orbital debris. The historical growth of objects in low Earth orbit (LEO) is shown in Figure 1.1. Compared to the non-mitagation results in Figure 1.3, post-mission disposal (PMD) is very effective in reducing orbital debris in LEO.

The creation of orbital debris is such a concern to scientists and engineers because of a detrimental circumstance known as the Kessler syndrome [1]. The Kessler syndrome predicts that orbital debris will create more orbital debris. With the creation of orbital debris the risk of collisions increases, leading to more orbital debris, a cascading effect. Figure 1.2 shows the prediction of the growth of orbital debris given no mitigation measures [2]. The analysis was conducted by Liou using 100 Monte Carlo simulations of NASA's long-term orbital debris evolutionary model, LEGEND. Obviously removing debris already in Earth's orbit would virtually mitigate all risk to spacecraft, but such a solution would be

prohibitively expensive. A much more realistic solution is to plan for disposal in 


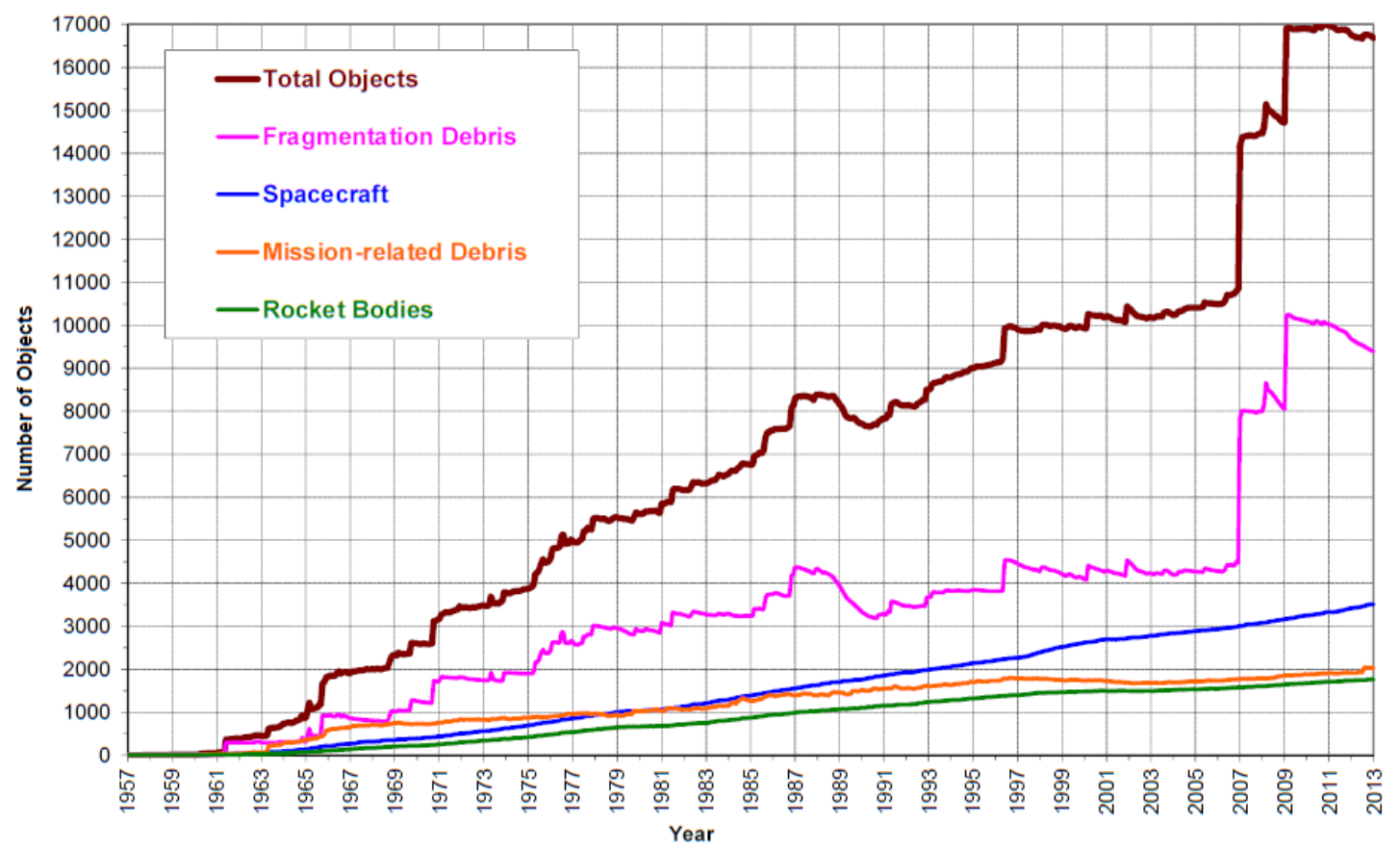

\section{Figure 1.1: Historical orbital debris population by debris type [3].}

future spacecraft while early in the design phase. For bodies in LEO this means de-orbiting the spacecraft after the end of its useful life.

The National Aeronautics and Space Administration (NASA) has set forth technical standards for de-orbiting spacecraft in the LEO regime within 25 years after end of mission, typically known as NASA's 25 year rule [4] [5] [6]. Typically, compliance with this set of requirements is achieved by either a de-orbiting propulsive maneuver or by allowing atmospheric drag to slowly lower altitude until reentry is achieved. Since drags acts in opposition of the velocity vector, energy is removed from the orbit and the altitude is lowered. Although natural spacecraft decay is possible, accelerating the decay is beneficial to the orbital debris environment. Liou shows, in Figure 1.3, the predicated evolution of the orbital debris environment given certain post-mission disposal and active debris removal (ADR) situations [2]. The first line represents that $90 \%$ of spacecraft 


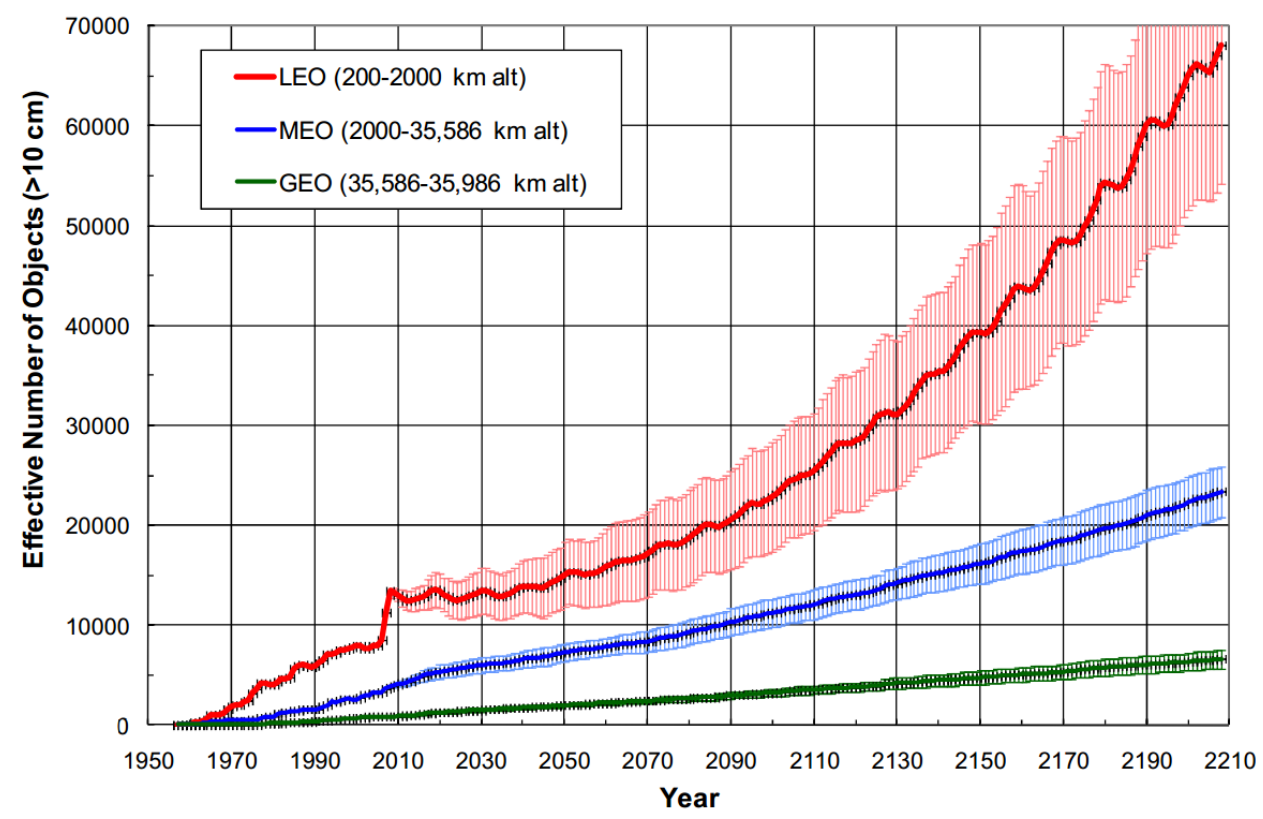

Figure 1.2: Prediction of the orbital debris population by altitude regime [2]. Mean represented with solid color line, shown with $1 \sigma$ standard deviation.

quickly de-orbit themselves. The second and third lines include, in addition to PMD, that each year the largest 2 and 5 pieces of orbital debris, respectively, are disposed of starting in 2020. The results, mean of 100 Monte Carlo simulations, of the LEO analysis can be seen in Figure 1.3. It should be noted that PMD alone does not solve the orbital debris. Some form of ADR is required to prevent a cascading orbital debris environment, although such discussion is beyond the scope of this project.

The ballistic coefficient is give by [7]:

$$
B C=\frac{m}{C_{D} A}
$$

where $m$ is the spacecraft's mass, $A$ is the area of the spacecraft facing the velocity vector, and $C_{D}$ is the coefficient of drag which will be discussed in Section 2.2.2. Decreasing a spacecraft's ballistic coefficient (which is inversely proportional to 


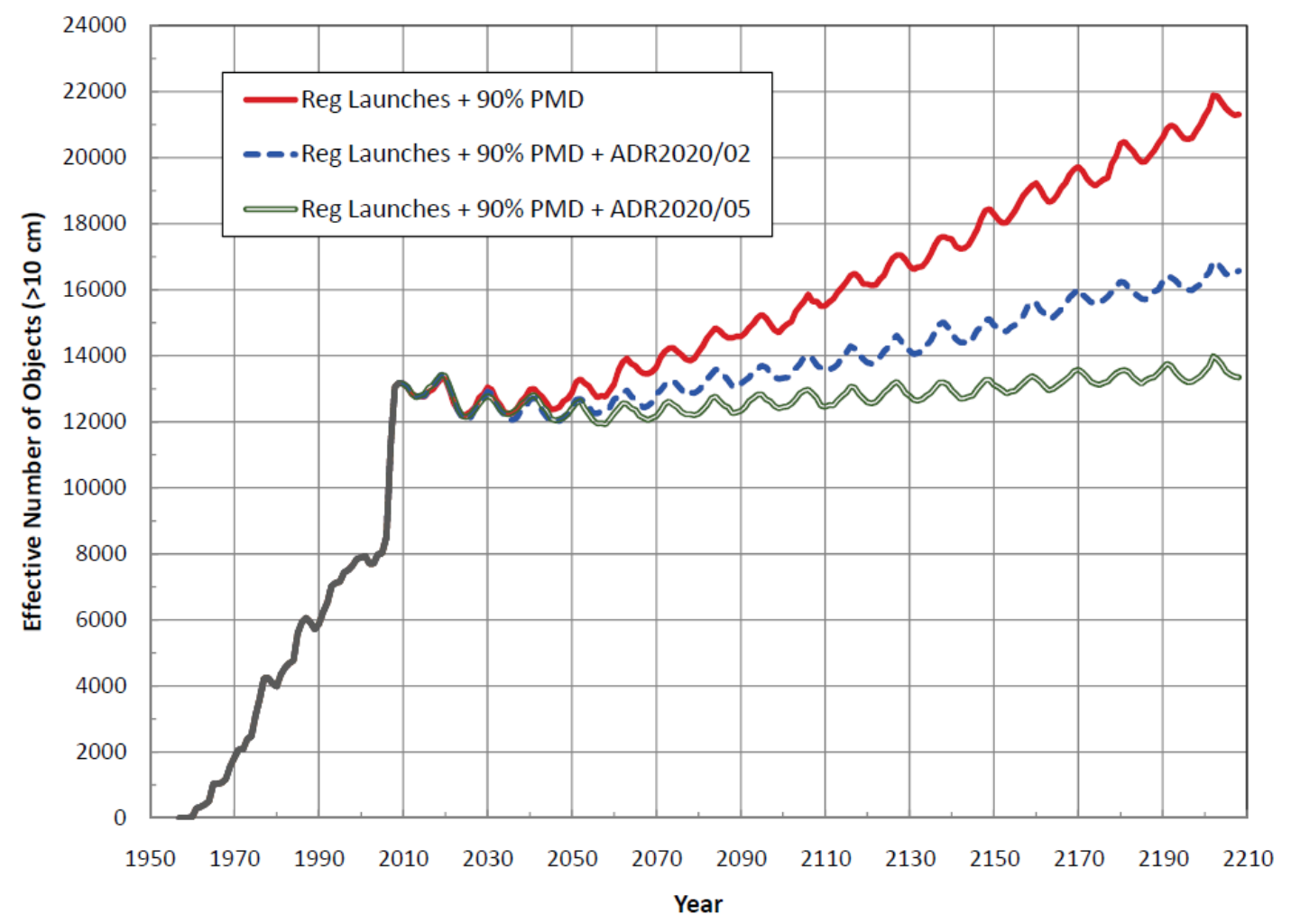

Figure 1.3: Analysis of the growth of the orbital debris environment [2].

its area to mass ratio) will decrease its orbital lifetime.

Presented here is a low-mass inflatable balloon-like device that increases the cross-sectional area and thusly decreasing orbital lifetime. More specifically, analysis will cover an inflatable de-orbit device, which is sized to be used for CubeSats. A CubeSat is nanosatellite class spacecraft, a single $1 \mathrm{U}$ CubeSat has a mass of no more than $1.33 \mathrm{~kg}$ and is a cube with sides of $10 \mathrm{~cm}$ [8]. CubeSats have been shown to be a low-cost platform for space technology demonstrations [9]. Since advanced methods of PMD is in its infancy the author believes that a successful CubeSat de-orbiting mission would allow for the technology to be used on a larger scale. 


\section{Orbital Dynamics}

Before much discussion is given to de-orbiting spacecraft, a review of orbital dynamics is in order. All bodies in a Keplerian orbit rely on gravity to maintain their orbit. The force of gravity is given by Newton's law of universal gravitation:

$$
F=G \frac{m_{1} m_{2}}{r^{2}}
$$

where $G$ is the gravitational constant, $m_{1}$ and $m_{2}$ are the masses of the two bodies, and $r$ is the distance between the two bodies. Typically in astrodynamics the standard gravitational parameter, $\mu$, is used to replace the product of the gravitational constant and the mass of the larger body. From this the acceleration, $a$ of gravity in vector form is given by [10]:

$$
\vec{a}=-\frac{\mu}{r^{3}} \vec{r}
$$

In a Keplerian system a satellite continues to orbit its parent body undisturbed by other forces. In reality a satellite is under a multitude of other accelerations such as: non-uniform gravity fields, solar radiation pressure, atmospheric drag, magnetic fields, and gravity effects from other bodies (n-body effects) to name the most prominent. These perturbing accelerations act in addition to the central gravity force and must be accounted for when calculating the trajectory of a spacecraft. Cowell's formulation is conceptually the simplest way to calculate 
the trajectory of a satellite, and is the summation of all accelerations acting on the body [7]:

$$
\vec{a}=-\frac{\mu}{r^{3}} \vec{r}+\sum \vec{a}_{\text {perturbations }}
$$

This equation is then fed into a numerical ordinary differential (ODE) solver; Runge-Kutta 4-5 for example.

How much a perturbation affects an orbiting spacecraft is a function of many parameters. Figure 2.1 shows approximate accelerations for selected major perturbations. Drag is mainly affected by the spacecraft's area to mass ratio and the local atmospheric density. Figure 2.1 depicts an area to mass ratio of 0.005 $\mathrm{m}^{2} / \mathrm{kg}$ and "moderate" [11] solar conditions. Solar radiation pressure, a force caused by the reflection or absorption of light by the spacecraft's surfaces, is also quite dependent on area to mass ratio, but is essentially independent of altitude. N-body effects, gravitational forces from other astronomical bodies, are largely independent of altitude. The effects of Earth's oblateness, in the form of J2 and higher terms, is dependent on altitude and position over the Earth.

\subsection{Non-uniform Gravity Field of Earth}

In a typical LEO environment the largest perturbation will be due to the Earth's gravity field. Since Earth is not a perfect sphere, as shown by its aspherical gravitational potential, the gravitational acceleration experienced by an orbiting spacecraft is not only varying in magnitude but also in direction. Due to the fact that Earth is spinning, centrifugal forces cause the planet to bulge along the equator [10]. Zonal harmonics are defined by central body's mass distribution along bands of latitude. The zonal harmonic about a planet's equator is known 


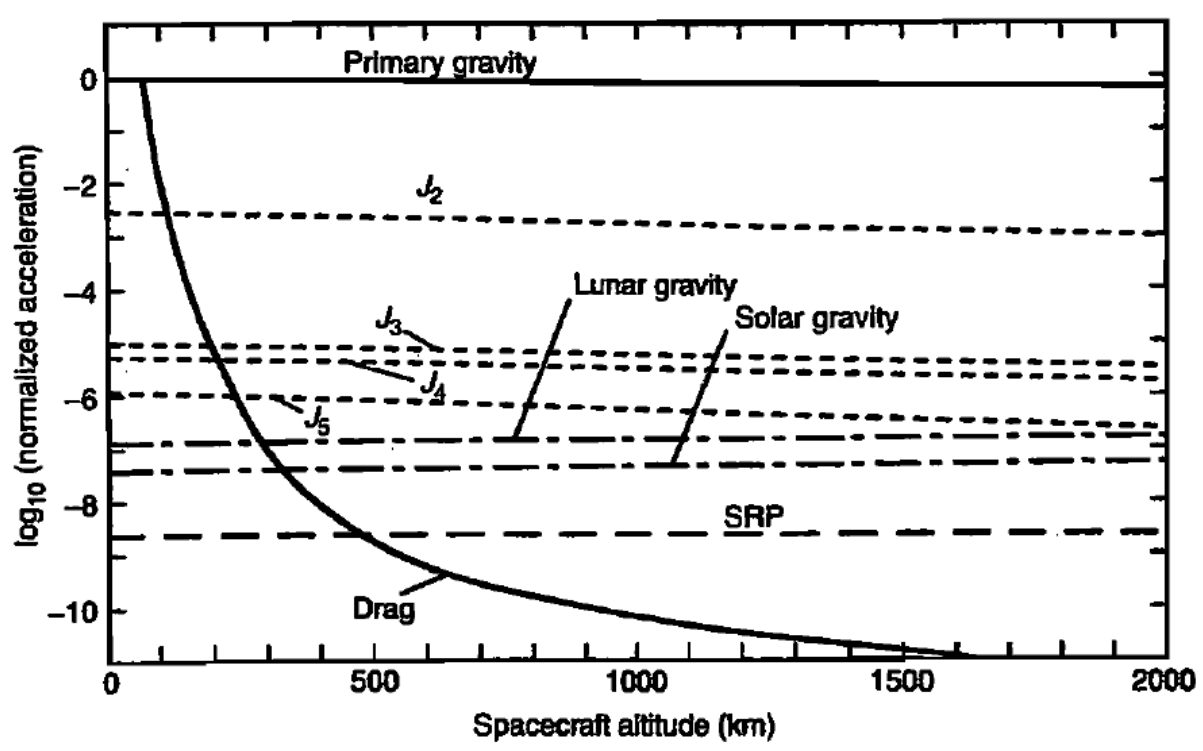

Figure 2.1: Magnitude of several different orbital perturbations compared to the acceleration from Earth, versus orbital altitude [11].

as the second zonal harmonic $\left(J_{2}\right)$, and is several orders of magnitude greater than the next greatest harmonic term $J_{3}[7]$, as seen in Figure 2.1. Other harmonic terms are those defined by longitude bands known as sectorial harmonics, and tesseral harmonics which use both latitude and longitude in a checkerboard-like array about the central body.

Since the $J_{2}$ harmonic is by far the largest aspherical gravity potential perturbation, it will be explained here but it is important to note that the calculation of higher-order zonal harmonic terms as well as sectorial and tesseral harmonic terms are very similar. In this calculation the acceleration will be in the Earth-Centered, Earth-Fixed (ECEF) reference frame, as opposed to the Earth-Centered Inertial (ECI) frame where the orbit is propagated. The difference between the two is that the ECEF frame rotates with Earth, whereas the ECI is fixed in inertial space, shown in Figure 2.2. Both frames are fixed in the "K" direction, defined by the north pole. In the ECI frame the "I" direction is along the Equator and 


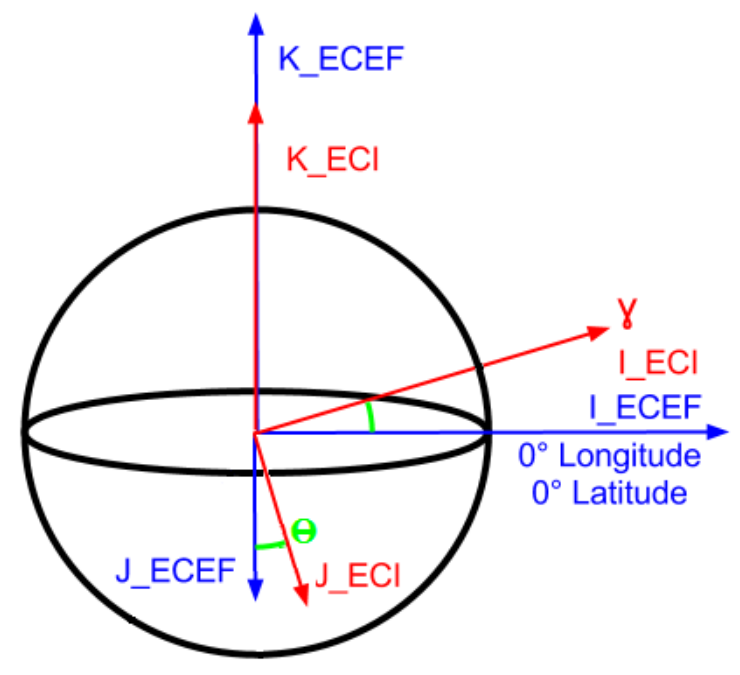

Figure 2.2: A visual of the ECI and ECEF reference frames [12].

always points towards the First Point of Aries, with the "J" component on the Equator normal to the "I" and "K" directions. In the ECEF frame the "I" and "J" componets are orientated at the intersection of the Equator and the Prime Meridian and Equator and 90 degrees West, respectively. The aspherical potential in the ECEF reference frame for the second zonal harmonic is given by [7]:

$$
R_{2}=-\frac{3 J_{2} \mu}{2 r}\left(\frac{R_{\oplus}}{r}\right)^{2}\left(\sin ^{2}(\phi)-\frac{1}{3}\right)
$$

where $J_{2}$ is the nondimensional second zonal coefficient, $R_{\oplus}$ is the radius of Earth, $r$ is the magnitude of the spacecraft radius vector, and $\phi$ is the geocentric latitude of the spacecraft. Since latitude is defined by:

$$
\phi=\arcsin \left(\frac{r_{K}}{r}\right)
$$


Equation 2.4 can be rewritten as:

$$
R_{2}=-\frac{3 J_{2} \mu R_{\oplus}^{2} r_{K}^{2}}{2 r^{5}}+\frac{J_{2} \mu R_{\oplus}^{2}}{2 r^{3}}
$$

Taking the partial derivative of this term with respect to each direction $(I, J$, and $K$ ) will yield the acceleration in that direction, in the ECEF frame.

$$
a_{I}=\frac{\partial R_{2}}{\partial r_{I}}=-\frac{3 J_{2} \mu R_{\oplus}^{2} r_{I}}{2 r^{5}}\left(1-\frac{5 r_{K}^{2}}{r^{2}}\right)
$$

likewise,

$$
\begin{aligned}
& a_{J}=-\frac{3 J_{2} \mu R_{\oplus}^{2} r_{J}}{2 r^{5}}\left(1-\frac{5 r_{K}^{2}}{r^{2}}\right) \\
& a_{K}=-\frac{3 J_{2} \mu R_{\oplus}^{2} r_{K}}{2 r^{5}}\left(3-\frac{5 r_{K}^{2}}{r^{2}}\right)
\end{aligned}
$$

These equations are combined into a vector to form the acceleration, shown:

$$
a_{J_{2}}=\left[\begin{array}{c}
a_{I} \\
a_{J} \\
a_{K}
\end{array}\right]
$$

Again, the process above is very similar for other terms given the aspherical potential for that harmonic.

\subsection{Atmospheric Drag}

Acceleration due to drag acts in opposition to the velocity vector, slowing a spacecraft down and lowering its orbital energy. Drag is a function of the spacecrafts 
velocity $V$, area, mass, shape (in terms if its $C_{D}$ ), and local atmospheric density $\rho$, and is given by [7]:

$$
\vec{a}_{\text {Drag }}=-\frac{\rho V^{2} C_{D} A}{2 m} \hat{V}
$$

Atmospheric density and the coefficient of drag will be discussed in later

sections. An important distinction that should be made here is the difference between inertial and relative velocity. Typically in numerical calculation the inertial velocity is used to propagate the orbit. Here the velocity of the spacecraft relative to the local atmospheric particles is needed, the two velocities can be related by:

$$
\vec{V}_{\text {relative }}=\vec{V}_{\text {inertial }}-\vec{\omega}_{\oplus} \times \vec{r}
$$

where $\mathrm{r}$ is the position vector of spacecraft, and $\vec{\omega}_{\oplus}$ is the rotational velocity vector of Earth. In this project it is assumed that Earth's atmosphere is rotating with the planet, and upper atmospheric winds are neglected.

\subsubsection{Atmospheric Density}

The density of the upper-atmosphere is typically the most difficult parameter to predict [7]. It not only varies through many orders of magnitude throughout a range of altitudes, but also orders of magnitude at a single altitude [11] due to variations in solar conditions, Earth's magnetic field, and local molecular composition [7]. Although there are many atmospheric models, only two will be discussed here.

The 1976 U.S. Standard Atmosphere (USSA 1976) is a simple model that is 
independent of time, solar conditions, magnetic conditions, and other parameters that other models utilize. Due to the model's simplicity, it is often used for firstorder analysis. The model relies on a look-up table for values of scale height $H$, base density $\rho_{o}$, and base altitude $h_{o}$ for a given altitude range $h_{l}$, and is given by Equation 2.13. The look-up table for the USSA 1976 model can be found in Table A.1.

$$
\rho=\rho_{o} \exp \left[-\frac{h_{l}-h_{o}}{H}\right]
$$

The Naval Research Laboratory's model, NRLMSISE-00, is very popular and relatively accurate throughout many different altitudes [7]. The model takes time, latitude, longitude, and several solar flux (F10.7) and geomagnetic index values (ap) and produces density and temperature values, as well as selected species concentrations. The NRLMSISE-00 model is freely available.

The U.S. Standard Atmosphere is bounded by the NRLMSISE-00 model at solar minimum and the NRLMSISE-00 at solar maximum as seen in Figure 2.3. 


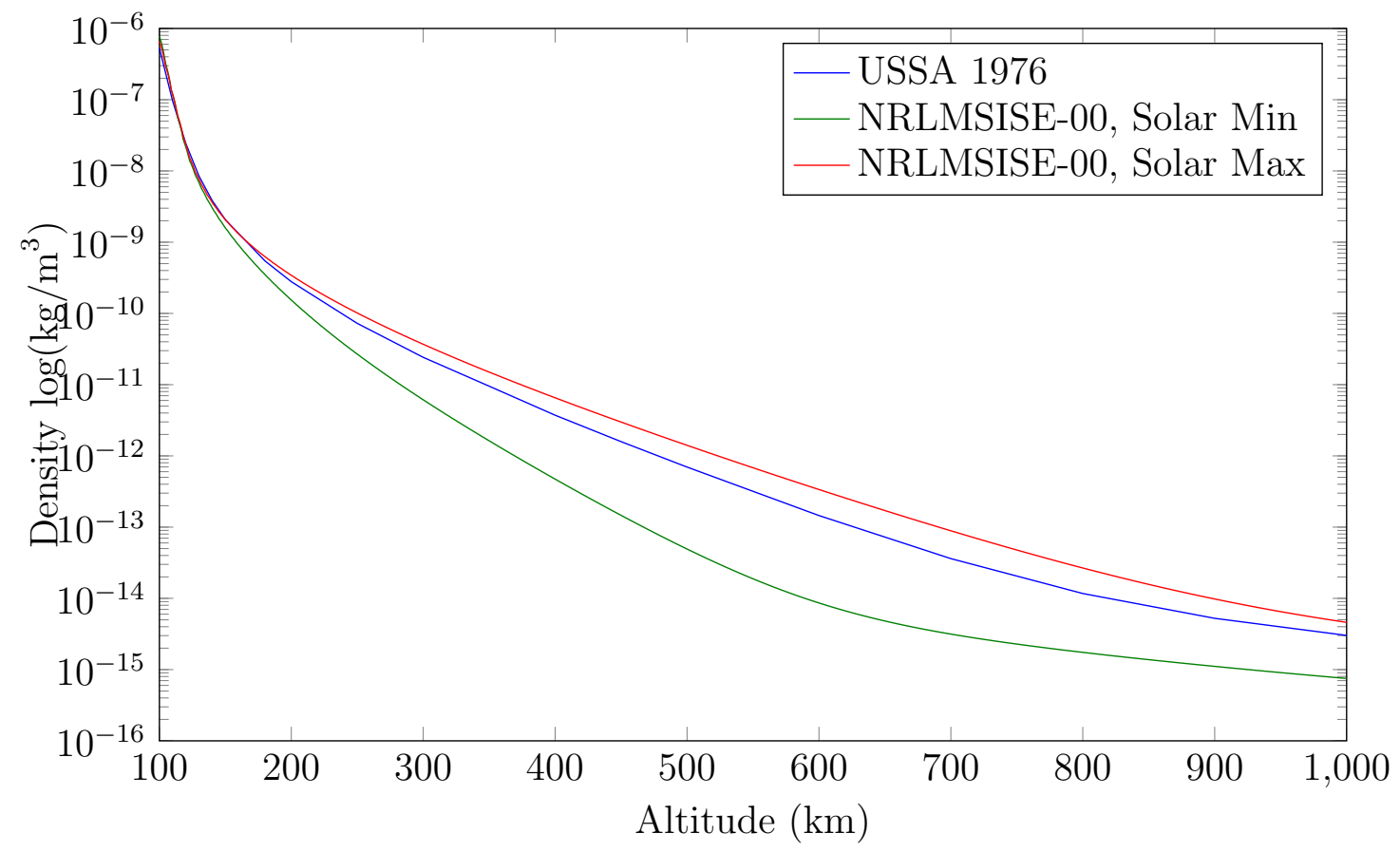

Figure 2.3: A comparison of the U.S. Standard Atmosphere and the NRLMSISE-00 model at solar maximum and solar minimum, for a range of altitudes.

For inputs to the NRLMSISE-00 model, it was assumed that the F10.7 solar flux was bounded between 70 and 200 and the geomagnetic index was bounded by 5 and 30 . The trend of these two parameters followed a sinusoidal curve for an 11 year cycle, a contour plot of densities (on a log scale) based on the number of years since solar minimum is seen in Figure 2.4. The latitude and longitude input to the NRLMSISE-00 were chosen at random. 


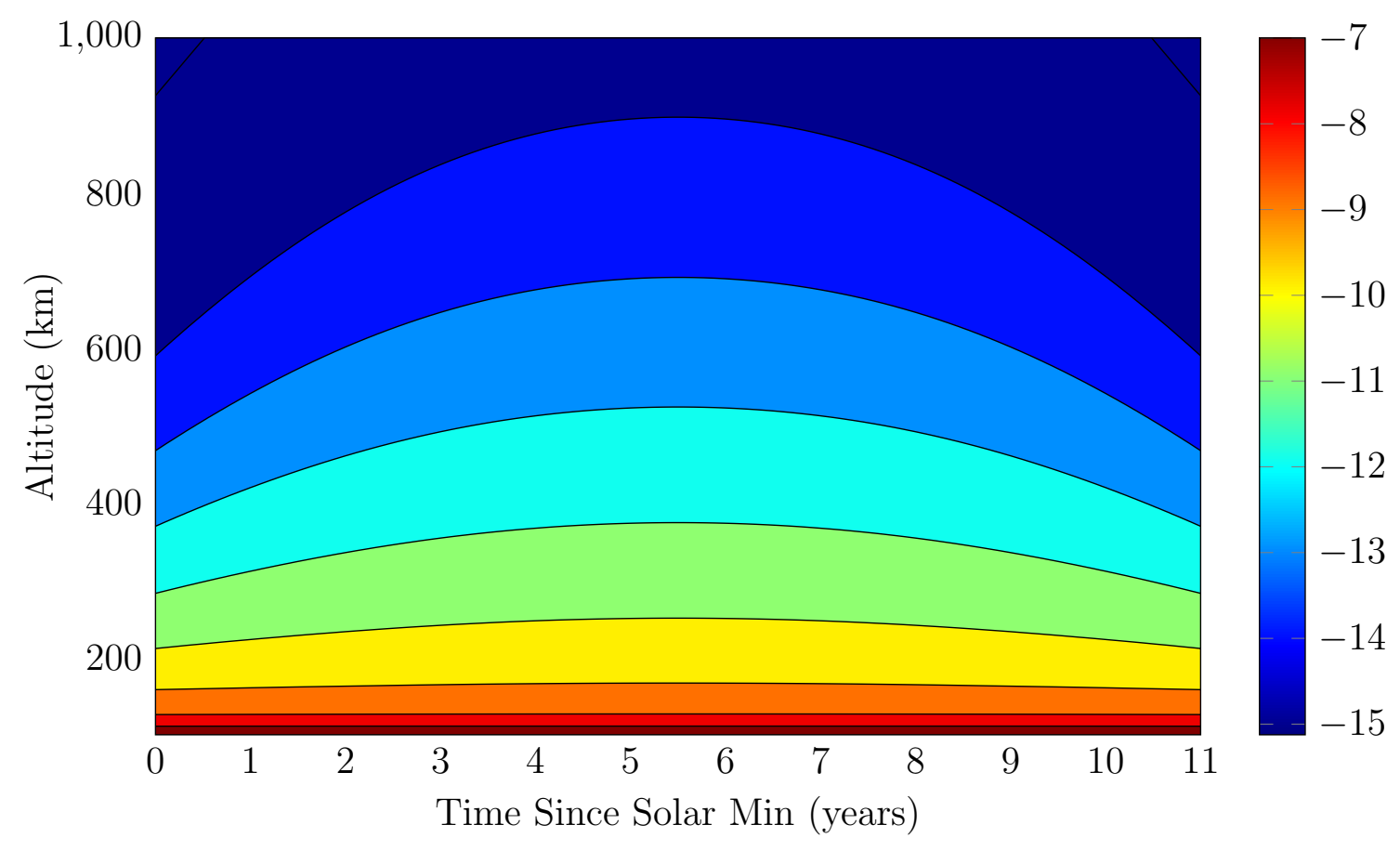

Figure 2.4: Contour of atmospheric density $\left(\mathrm{kg} / \mathrm{m}^{3}\right)$ based on altitude and years since the last solar minimum, log scale.

\subsubsection{Coefficient of Drag}

Historically a coefficient of drag of 2.2 has been used[13] when propagating a spacecraft's orbit. It has been decided that for this project a more accurate coefficient of drag model would be developed. For this analysis the spacecraft will be assumed to be spherical to simplify the calculations.

A spacecraft in a low Earth orbit encounters the flow of atmospheric particles that is in the rarefied and transitional regimes. Many attempts to derive a spacecraft's coefficient of drag are at the mercy of the accuracy of two-line element (TLE) for state vector data which often and cannot capture high-frequency changes in the local atmospheric density due to changing solar and geomagnetic conditions [14]. Recently with spacecraft containing high-accuracy accel- 
eratomers, such as Gravity Recovery and Climate Experiment (GRACE), it is possible to obtain in situ measurements of the spacecraft's coefficient of drag. An estimation of drag coefficients based on shape and altitude can be seen in Figure 2.5, these curves are calculated from a combination of an analytical diffuse reflection model and from values measured in orbit [15]. Typical analyses for calculating spacecraft drag coefficient include particle-based numerical methods or simplifying the spacecraft geometry and using closed-form analytical methods.

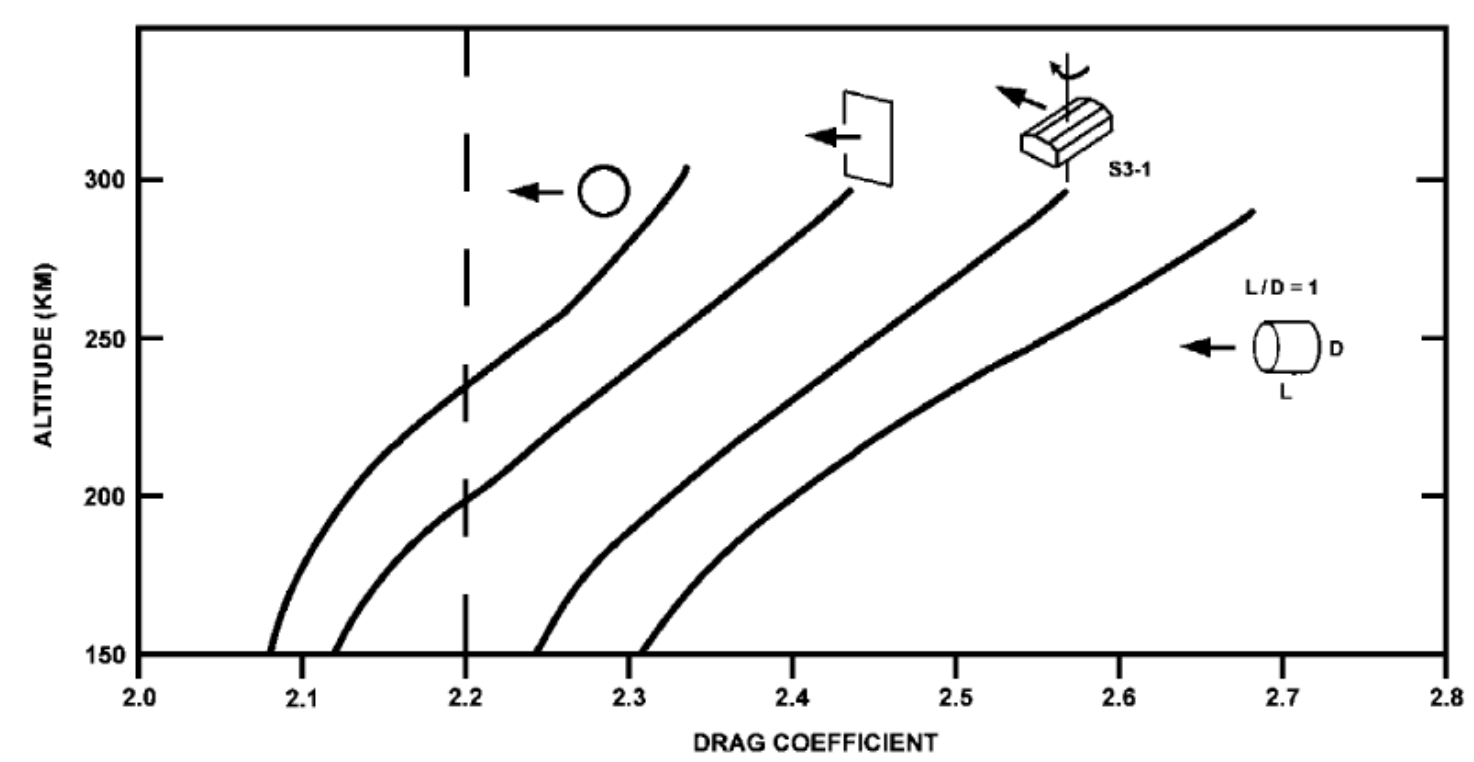

Figure 2.5: Estimation of drag coefficient based on body shape and altitude. [15].

The mean free path, $\lambda$, is the parameter which gives the average distance a molecule travels before colliding with another molecule, and is given by [16]:

$$
\lambda=\frac{1}{\sqrt{2} \pi d^{2} n}
$$

where $d$ is the diameter of an individual particle in the flow and $n$ is the number density of the flow. The Knudsen number relates the mean free path to a char- 
acteristic length, $L$, in this case the characteristic length would be that of the spacecraft.

$$
K_{N}=\frac{\lambda}{L}
$$

Typically for flows with a Knudsen number that is less than 0.1, Navier-Stokes equations are valid and computational fluid dynamics (CFD) is solver method of choice [17]. For Knudsen number greater than 10 [18] collisionless Boltzmann equations become the preferred governing equations and the problem is usually solved via test particle Monte Carlo (TPMC) method. The direct simulation Monte Carlo (DSMC) method is a particle-based method used to numerically solve gas dynamics problems [19]. DSMC is valid for flows with all Knudsen numbers, but is most computationally efficient at calculating transitional regime flows, with Knudsen numbers between 0.1 and 10. Figure 2.6 shows the distribution of flow regimes with their typical solvers.

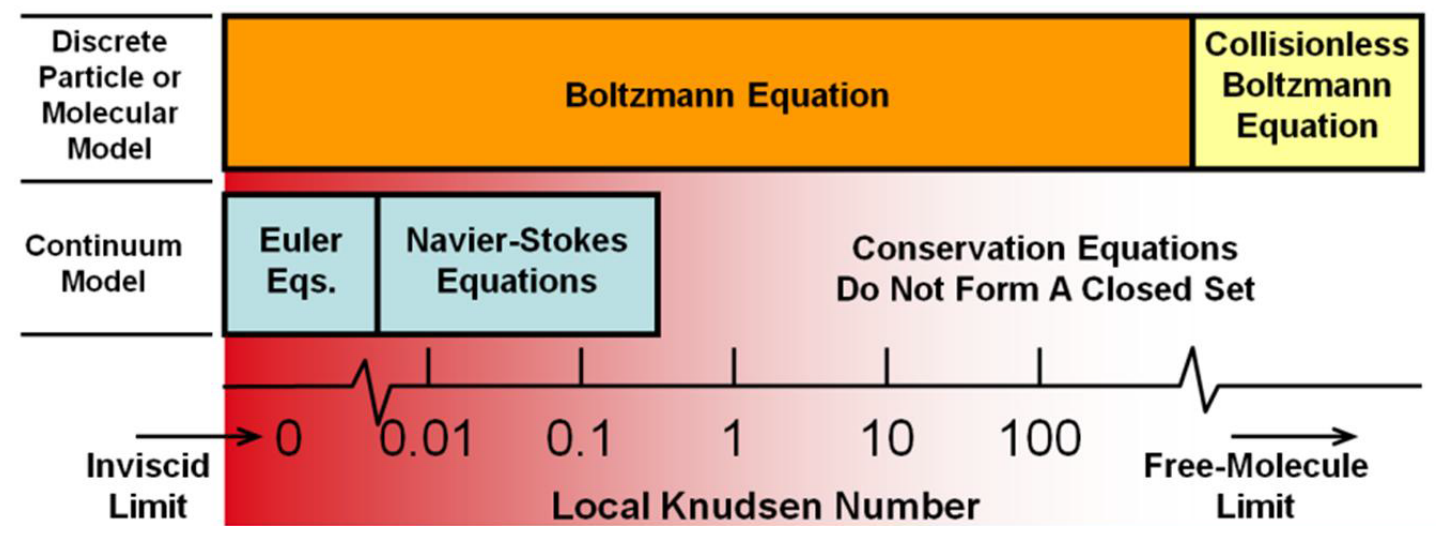

Figure 2.6: A range of Knudsen numbers showing associated valid solvers [20].

For a spacecraft of a simple shape [21] and in a fully rarefied environment closed-form solutions exist to quickly calculate a spacecraft's coefficient of drag, 
given some input parameters. For spacecraft in lower Earth orbits, the flow around the body is transitional, and intermolecular collisions become very crucial and analytical models break down.

The model of how individual particles reflect off of a spacecraft's surface is important. The gas-surface interaction is the main uncertainty in calculating the coefficient of drag [19]. As shown in Figure 2.7 there are three main ways a particle can be reflected off a surface: specular, diffuse, and quasi-specular.

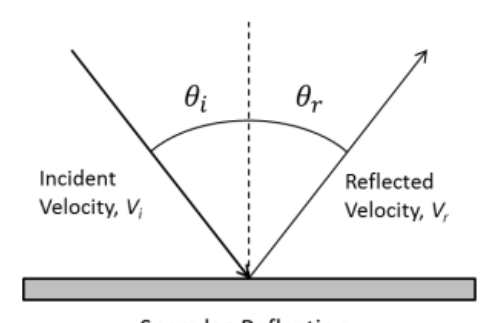

Specular Reflection

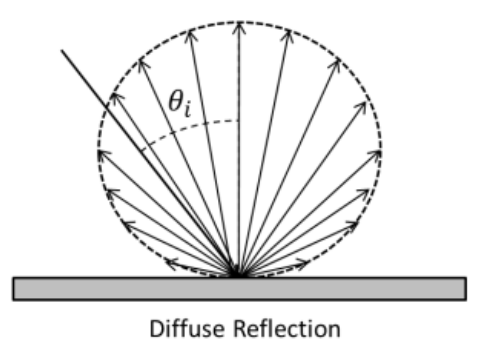

Diffuse Reflection

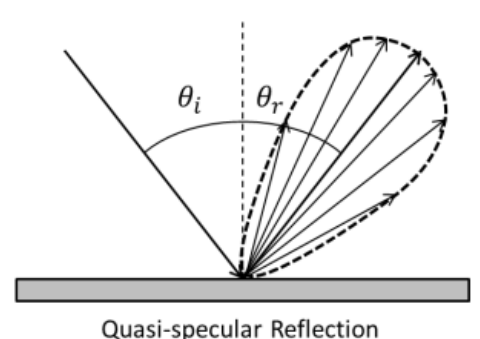

Quasi-specular Reflection

Figure 2.7: A visual representation of fully specular, diffuse, and quasispecular refection [19].

Specular reflection happens when an incident particle strikes a surface and is reflected at an angle equal to the incident angle, relative to the surface. The difference of specular reflection on a sphere and flat plate, perpendicular to the flow, is shown in Figure 2.8. 

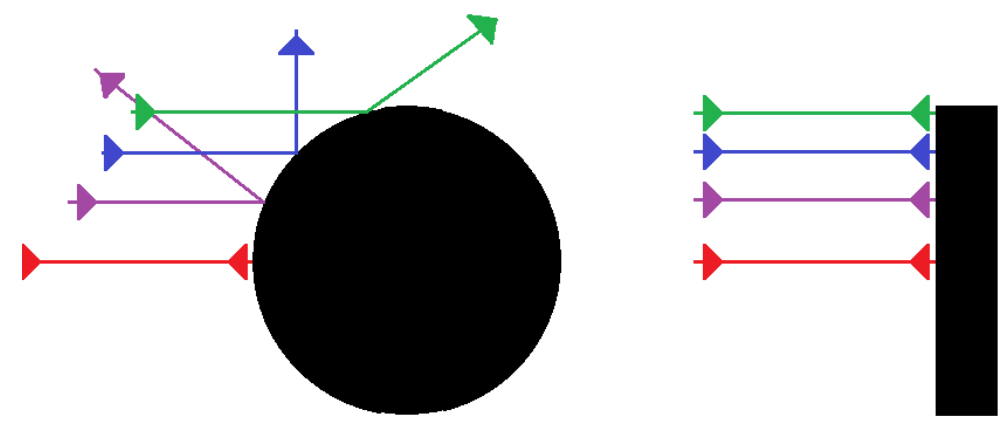

Figure 2.8: A visualization of fully specular refection of a sphere (left) and flat plate (right).

\section{Fully Diffuse Reflection}

Assuming a completely diffuse reflection, as opposed to a fully specular or quasispecular refection as compared in Figure 2.7, a closed-form solution for drag of a spherical object is given by [19]:

$$
C_{D}=\frac{4 s^{4}+4 s^{2}-1}{2 s^{4}} \operatorname{erf}(s)+\frac{2 s^{2}+1}{\sqrt{\pi} s^{3}} \exp ^{-s^{2}}+\frac{2 \sqrt{\pi}}{3 s} \sqrt{\frac{T_{s}}{T_{\infty}}}
$$

Where erf is the error function, as shown in Equation 2.17, $T_{s}$ is the surface temperature, $T_{\infty}$ is the free-stream flow temperature, and $s$ is the ratio of bulk velocity to the most probable speed, $v_{m p}$, shown in Equation 2.18.

$$
\begin{gathered}
\operatorname{erf}(x)=\frac{2}{\pi} \int_{0}^{x} \exp ^{-t^{2}} d t \\
s=\frac{V}{v_{m p}}
\end{gathered}
$$

The most probable speed of a Maxwell-Boltzmann distribution is shown in 
Equation 2.19.

$$
v_{m p}=\sqrt{\frac{2 k_{B} T_{\infty}}{m}}
$$

Where $k_{B}$ is Boltzmann's constant and $m$ is the mean molecular mass of the free-stream particles.

The fully diffuse reflection, also sometimes known as the Maxwellian model, is the simplest of the reflection models presented here. This model assumes complete accommodation [19], meaning that there is no exchange of energy between the surface of a body and an incident particle. That is, when an atmospheric particle strikes a surface it is reflected with the same energy.

\section{Diffuse Reflection with Incomplete Accommodation}

The diffuse reflection with incomplete accommodation (DRIA) model assumes that an particle is reflected diffusely, but with a loss of accommodation [16].

$$
C_{D}=\frac{4 s^{4}+4 s^{2}-1}{2 s^{4}} \operatorname{erf}(s)+\frac{2 s^{2}+1}{\sqrt{\pi} s^{3}} \exp ^{-s^{2}}+\frac{2 \sqrt{\pi}}{3} \frac{U_{r}}{U_{i}}
$$

The $U_{r} / U_{i}$ term is the ratio of reflected to incident energy of a particle striking a surface.

$$
\frac{U_{r}}{U_{i}}=\sqrt{\frac{2}{3}} \sqrt{1+\alpha\left(\frac{E_{s}}{E_{i}}-1\right)}
$$

The accommodation coefficient, $\alpha$, will be discussed later in this section. The energy ratio of the surface and the incident particle is equal to the ratio of their 
temperatures.

$$
\frac{E_{s}}{E_{i}}=\frac{T_{s}}{T_{k}, i}
$$

Finally, the kinetic temperature of the incident particle is given by

$$
T_{k, i}=\frac{m V^{2}}{3 k_{B}}
$$

The DRIA model is the most popular model, and has been shown to be very accurate up to a $500 \mathrm{~km}$ altitude, and possibly up to $800 \mathrm{~km}$ [19]. It has been shown that for spacecraft at $225 \mathrm{~km}$ the reflection of particles is $97 \%$ diffuse and $3 \%$ quasi-specular [15].

\section{Cercignani-Lampis-Lord Model}

At higher altitudes reflection becomes more quasi-specular. The CercignaniLampis-Lord (CLL) model takes into account differing normal and tangential accommodation coefficients [19]. For a spherical body the normal momentum co-

efficient, $\sigma_{n}$, and tangential momentum coefficient, $\sigma_{t}$, terms are necessary [16]. It is important to note that for other geometries, and for numerical calculation, the normal and tangential energy coefficient, $\alpha_{n}$ and $\alpha_{t}$ respectively may also be required. Also important to note is that although the total momentum and energy terms are coupled, the normal and tangential components are independent [21]. The drag coefficient using the CLL model is given:

$$
C_{D}=\frac{2-\sigma_{n}+\sigma_{t}}{2}\left(\frac{4 s^{4}+4 s^{2}-1}{2 s^{4}} \operatorname{erf}(s)+\frac{2 s^{2}+1}{\sqrt{\pi} s^{3}} \exp ^{-s^{2}}\right)+
$$




$$
\frac{2 \sigma_{n} \sqrt{\pi}}{3 s} \sqrt{\frac{T_{s}}{T_{\infty}}}
$$

As discussed later the CLL model will not be used in the analysis presented here. Though there will be no further discussion on the momentum accommodation coefficients, the author does believe that the CLL model is important enough to warrant mention. Figure 2.9 shows the difference between the three models discussed here, also shown is a fitted drag coefficient for the observed orbital decay of spherical bodies.

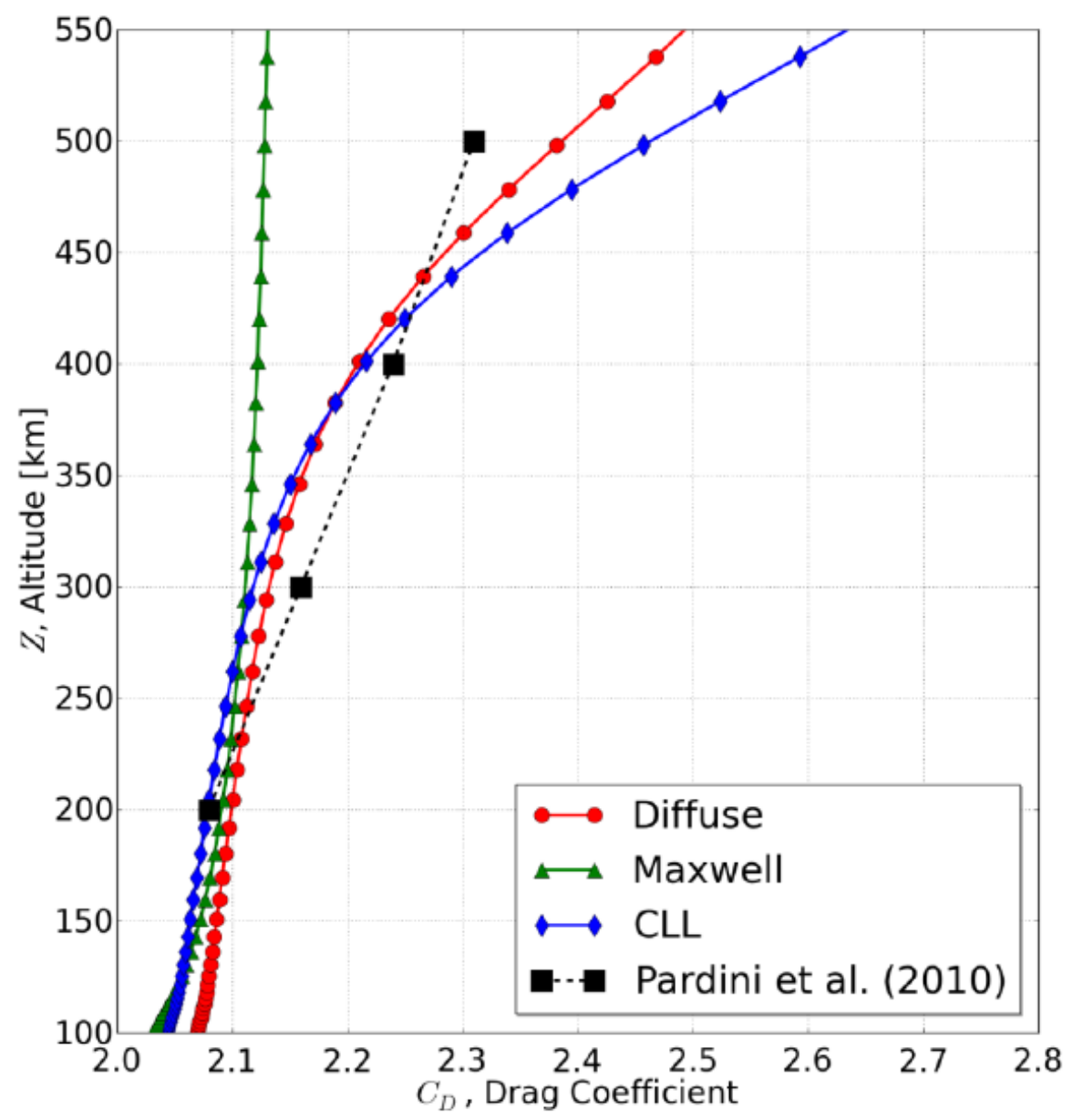

Figure 2.9: Drag Coefficient for Diffuse, DRIA, and CLL modes, and a curve from Pardini's observation [21]. 


\section{Direct Simulation Monte Carlo}

DSMC is a numerical method of modeling gas by using simulated gas molecules. Often, each simulated molecule represents many thousands if not millions of real molecules. The position of these represented molecules are propagated in time and intermolecular collisions and boundary condition interactions are calculated. The way the method calculates the collisions is based on particle physics, contrasting to CFD which attempts to solve fluids problems via Navier-Stokes equations $[17]$.

The particular DSMC software package that was utilized in this project is Dr. Bird's DS2V program, a two-dimensional/axially symmetric solver. The program requires inputs similar to CFD solvers such as flow field size, body shape, boundary conditions, and flow characteristics. Unlike typical CFD solvers however, additional inputs including species concentrations, accommodation coefficients, and surface/molecule and intermolecular collision reaction characteristics are needed. The program does not require a mesh, instead the program generates its own and updates the mesh using a built-in adaptive mesh refinement scheme. Also, since DSMC solutions are inherently transient DS2V adaptively adjusts the size of the time-steps used in calculation. The program has its own post-processor which displays information from the run in a user-friendly manner [17]. Select results from the DSMC calculations can be found in Table B.1. Note that the free-stream speed of an atmospheric particle is assumed to be $7700 \mathrm{~km} / \mathrm{s}$.

\section{Energy Accommodation Coefficient}

The accommodation coefficient is the parameter used to relate how much energy is transfered between an incoming atmospheric particle and a spacecraft's surface. 
By definition the accommodation coefficient is [22]:

$$
\alpha=\frac{E_{i}-E_{r}}{E_{i}-E_{s}}
$$

Which can also be written in terms of temperature:

$$
\alpha=\frac{T_{i}-T_{r}}{T_{i}-T_{s}}
$$

Since these components are impossible to measure on orbit, many attempts have been made to approximate the accommodation coefficient given in more convenient terms. One such formulation is given by Gooding [23].

$$
\alpha=\left(1-\theta^{\prime}\right) \alpha_{s}+\theta^{\prime}
$$

where $\theta^{\prime}$ is the portion of the spacecraft surface covered by an adsorbate, or atmospheric gas molecules, typically atomic oxygen.

$$
\theta^{\prime}=\frac{K P_{O}}{1+K P_{O}}
$$

The term $K$ is the Langmuir fitting parameter and will be assumed to be 4.98E$17 \mathrm{~m}^{3} \mathrm{~K}^{-1}$ for now [22], and $P_{O}$ is the partial pressure of atomic oxygen. In Equation 2.27 the adsorbate accommodation coefficient is:

$$
\alpha_{s}=\frac{2.4 \mu}{(1+\mu)^{2}}
$$

and $\mu$ is the ratio of average free stream particle molecular mass, $\bar{m}$ to the average 
molecular mass of a particle on the surface, $m_{s}[23]$.

$$
u=\frac{\bar{m}}{m_{s}}
$$

Pilinski gives another equation for the accommodation coefficient which is based on empirical satellite observations:

$$
\alpha=\frac{7.50 \times 10^{-17} n_{O} T_{i}}{1+7.50 \times 10^{-17} n_{O} T_{i}}
$$

where $n_{O}$ is the number density of atomic oxygen.

The final accommodation coefficient model presented here is by Mehta, McLaughlin, and Sutton [14]. This model is a modification of Equation 2.31, and has been shown to fit well with data from the GRACE mission.

$$
\alpha=\frac{\alpha_{s}+K P_{O}}{1+K P_{O}}
$$

In this model the Langmuir parameter is $4.86 \mathrm{E} 6 \mathrm{~Pa}$, and the term $\alpha_{s}$ is the same as shown in Equation 2.29.

\section{Results}

Given the same assumptions that were used in making the atmospheric density model (Section 2.2.1), results were obtained for a coefficient of drag. For low Knudsen numbers, where the flow is transitional DSMC results can be used. Above $250 \mathrm{~km}$ is where free molecular flow will be assumed to take place, here the DRIA model will be used.

For transitional flow DSMC simulations were conducted and a look-up table 
of drag coefficients can be interpolated between. The CLL model is used in the DSMC program with a momentum and energy accommodation coefficients of 1 . These value were held constant throughout the altitude range. The other flow parameters such as temperature and species densities were calculated using mean solar and geomagnetic conditions from the NRLMSISE-00 atmospheric model. Example velocity and pressure plots from a simulation at $100 \mathrm{~km}$ can be seen in Figure 2.10. The flow field is $-10 \mathrm{~m}$ to $4 \mathrm{~m}$ in the $\mathrm{x}$-direction and $0 \mathrm{~m}$ to $4 \mathrm{~m}$ in the y-direction. The flow field is weighted and axially symmetric about the y-axis, and a $1 \mathrm{~m}$ diameter sphere centered at the origin of the flow field. The velocity of the flow is considered to be the velocity of a body at altitude in a circular orbit. Note the well defined bow shock that is not found at high altitudes, seen in Figure B.1. The lack of a well-formed bow shock is indicative of rarefied flow.

Above $250 \mathrm{~km}$ the flow can be considered to be free molecular and the methods from Section 2.2.2 and Section 2.2.2 were used. Information for species concentrations, densities, and temperatures were obtained from the NRLMSISE-00 model. Being that this analytical method is much easier to compute a look-up table is not required and instead the algorithm is used directly inside of the orbit propagator. Example drag coefficients based on date and altitude can be seen in Figure 2.11, and a comparison between drag coefficients of mean, minimum, and maximum solar conditions in Figure 2.12. Both example plots assume free stream velocity is equal to circular orbital speeds at altitude.

The spike in Figure 2.12 at $250 \mathrm{~km}$ is due to the differing methods used to calculate the coefficient of drag. Recall that flow below $250 \mathrm{~km}$ is assumed to be transitional and DSMC results are used, while above the flow is assumed to be fully rarefied and the analytical methods are used. 

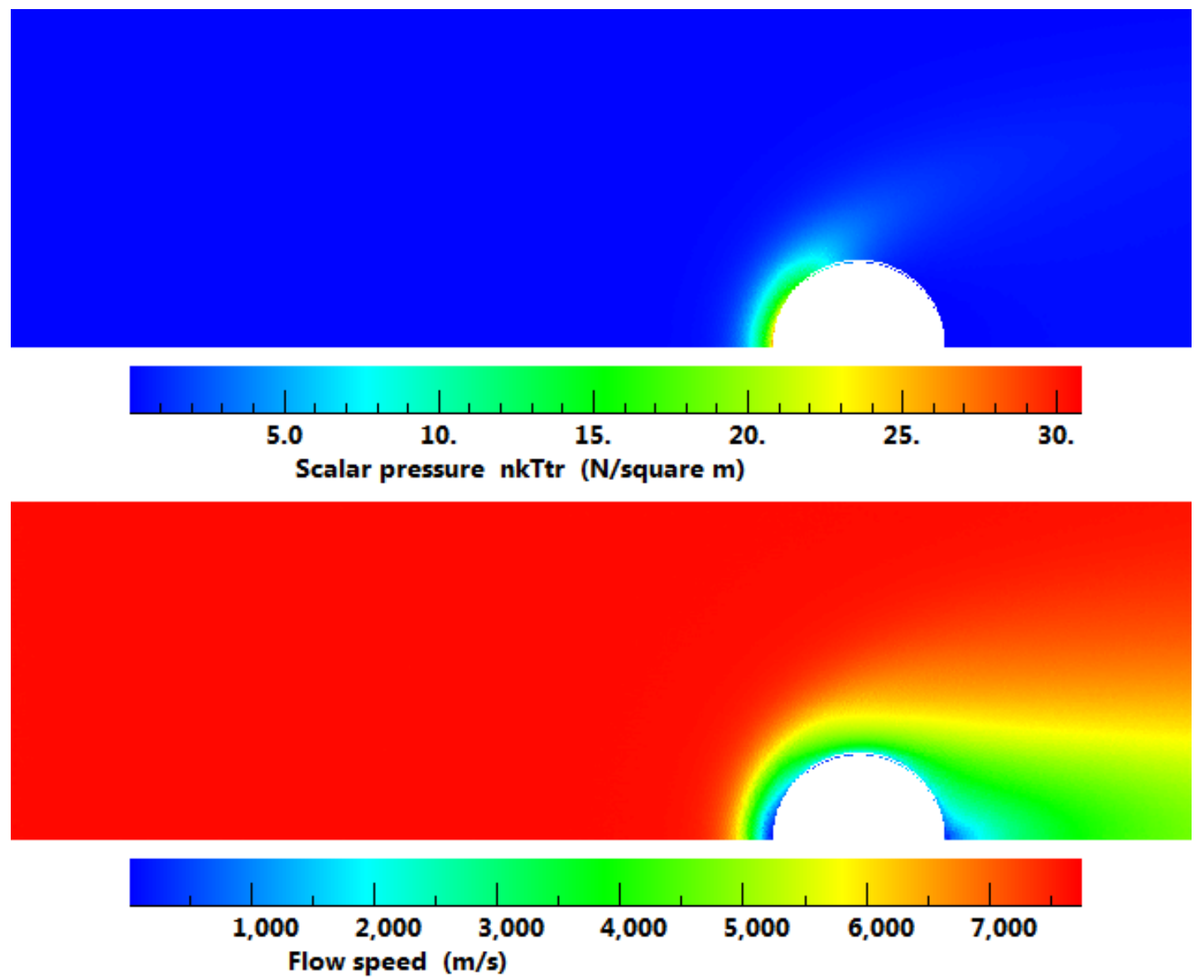

Figure 2.10: Flow pressure (top) and velocity (bottom) results from a DSMC simulation at $100 \mathrm{~km}$ altitude. 


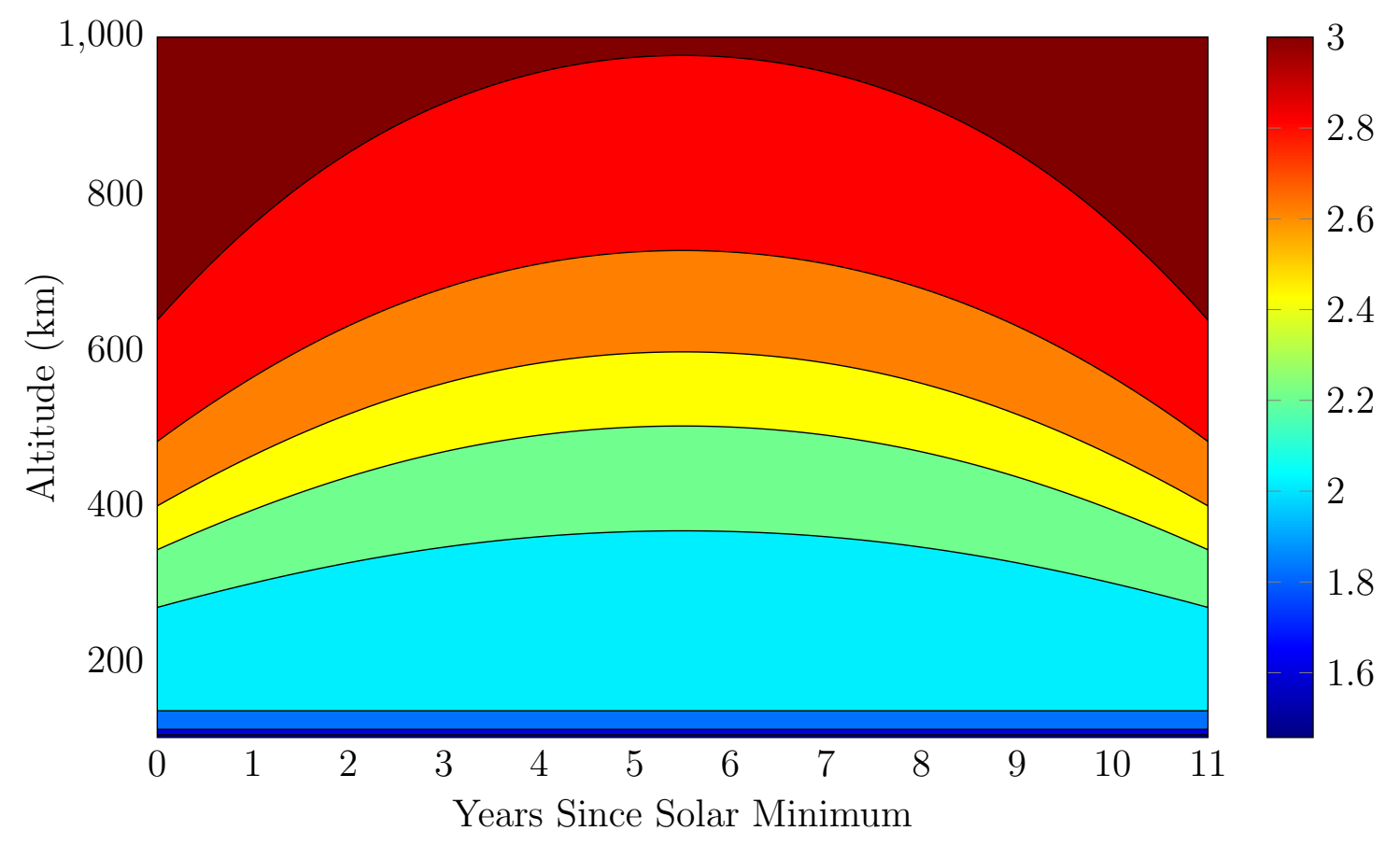

Figure 2.11: Contour of coefficient of drag values based on time since solar minimum and altitude.

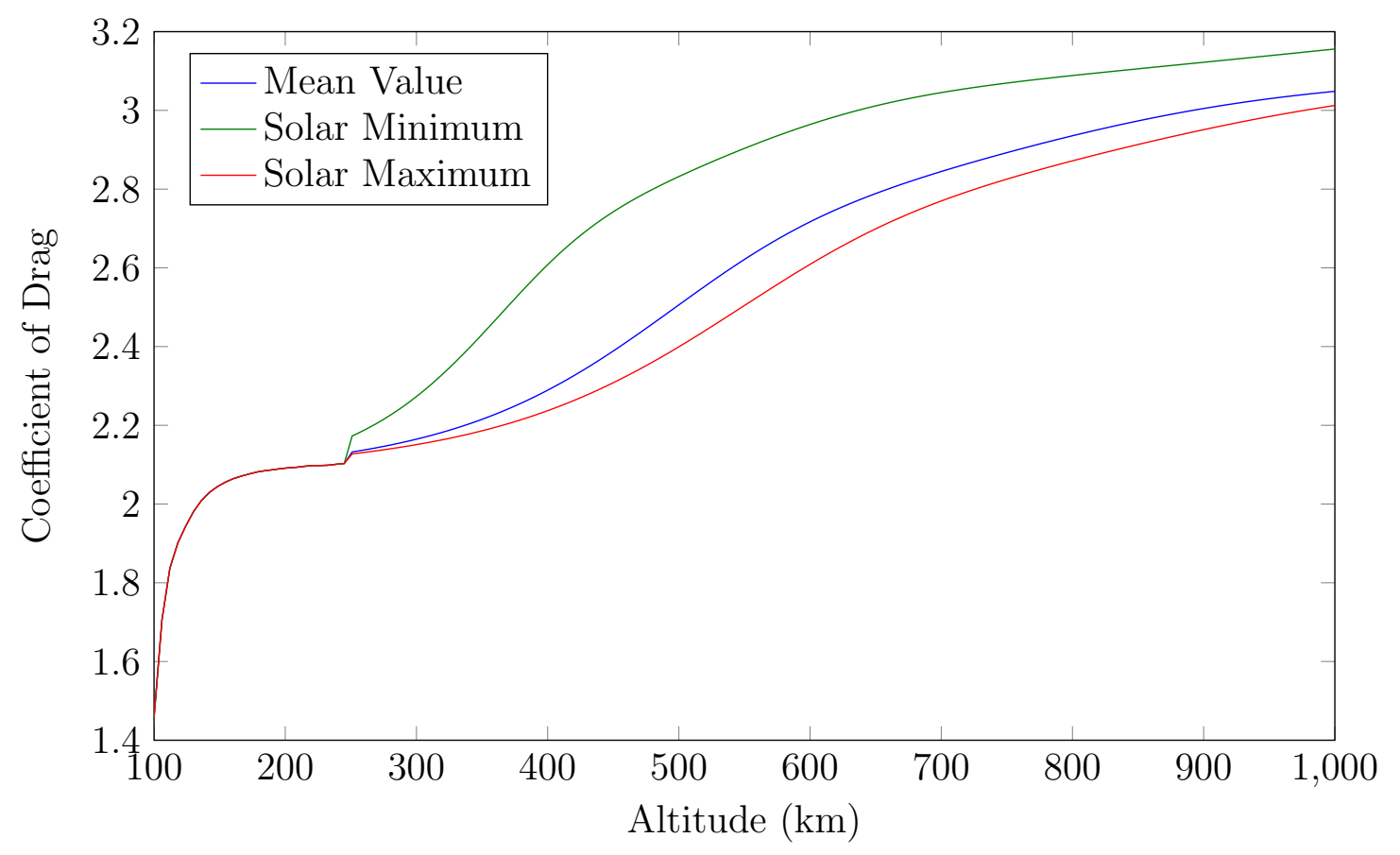

Figure 2.12: Coefficient of drag values based on altitude, taken at three solar conditions. 


\subsection{Solar Radiation Pressure}

A spacecraft also experiences a perturbation from sunlight, in terms of solar radiation pressure, and is given by [7]:

$$
\vec{a}_{S R P}=-\frac{P_{S R} C_{R} A}{m} \frac{\vec{r}_{s a t \odot}}{r_{s a t \odot}}
$$

The $P_{S R}$ term is the nominal solar radiation pressure at $1 \mathrm{AU}$ from the Sun, which will be assumed to be $9.55 \mu \mathrm{Pa}$. The coefficient of reflectivity, $C_{R}$, is a material property of the spacecraft and ranges from 0 to 2 . A coefficient of reflectivity of 0 means that the body is transparent and light simply passes through it, a coefficient of reflectivity of 1 means that all light is absorbed by the body, and a coefficient of reflectivity of 2 means that all light that strikes the body is reflected. It should be noted here that the area, $A$, here is different than the area in Equation 2.11 since this area is the amount of area facing the Sun. The position $r_{\text {sat } \odot}$ is the position of the spacecraft with respect to the Sun. Since a constant solar radiation pressure is assumed and the distance is not needed, only the unit vector will be utilized.

To get the position of the spacecraft with respect to the Sun, the position of the spacecraft in the ECI frame is needed as well as the position of the Earth with respect to the sun.

$$
r_{s a t \odot}=r_{\oplus s a t}-r_{\oplus \odot}
$$

Although analytical models exists to calculate Earth's position, NASA's Jet Propulsion Laboratory (JPL) maintains a database of space body ephemeris [24]. A look-up table of position vectors was obtained from JPL and a spline interpo- 
lation is performed to calculate the position vector needed on a given date.

Spacecraft in Earth orbit often pass behind the Earth, with respect to the Sun. When this happens the spacecraft is said to be in umbra (when the Sun in no visible at all) and penumbra (when the spacecraft is exposed to partial sunlight). In full umbra the solar radiation pressure is zero, and in penumbra the acceleration is lessened, assumed to be $50 \%$.

\subsection{Earth's Magnetic Field}

All bodies, with some charge, traveling through a magnetic field experience a force, the Lorentz force [25]:

$$
\vec{a}=q(\vec{V} \times \vec{B})
$$

The $q$ term is the charge of the spacecraft, $B$ is the local magnetic field of the Earth, and $V$ is the velocity of the spacecraft relative to the local magnetic field, see Equation 2.12. Since this is often a small force it is rarely used on first-order analysis but is presented here for completeness.

\subsection{N-Body Perturbations}

Accelerations due to the gravitational attraction of other astronomical bodies acts as a perturbation to a Keplerian orbit. Often this perturbation is referred to a $3^{\text {rd }}$ body effects, but since multiple other bodies may be taken into account the more generic term n-body is used as well. The equation for this perturbation is similar to the equation used for the acceleration due to Earth's gravity, in Equation 2.2. One distinction that must be made is by using Equation 2.2 directly would result 
in the acceleration vector of the spacecraft, but in the inertial frame of the $3^{r d}$ body. Therefore, the acceleration from a $3^{r d}$-body in the ECI frame is given by $[7]$.

$$
\vec{a}=\mu_{3}\left(\frac{\vec{r}_{s a t 3}}{r_{s a t 3}^{3}}-\frac{\vec{r}_{\oplus 3}}{r_{\oplus 3}^{3}}\right)
$$

The subscript 3 is used to denote the non-parent body. Therefore the terms $r_{\text {sat } 3}$ and $r_{\oplus 3}$ represent the position of the spacecraft with respect to the $3^{\text {rd }}$ body and the position of the Earth with respect to the $3^{\text {rd }}$ body, respectively. Since the position of the spacecraft with respect to the perturbing body is rarely known outright, it is often calculated by means of:

$$
\vec{r}_{s a t 3}=\vec{r}_{\oplus 3}-\vec{r}_{\oplus s a t}
$$

The acceleration from the Moon and Sun are, in a geocentric orbit, many order of magnitude greater than due to other astronomical bodies in our Solar System. The Sun since it has such a very large mass, and the Moon since it is so very close to a body in a geocentric orbit. 


\section{Current Work in Spacecraft Disposal}

In order to mitigate future orbital debris, and to comply with NASA's 25 year rule [4] [5] [6], many spacecraft engineers and operators are designing their LEO vehicles to de-orbit at end of life. For spacecraft in a geosynchronous orbit (GEO), as defined as a non-inclined circular orbit with a period of one day, NASA requirements state that the spacecraft be placed in a "graveyard" orbit. For GEO the graveyard orbit is $300 \mathrm{~km}$ above GEO [5], such that a decommissioned spacecraft does not reenter GEO within 100 years due to orbital perturbations [6]. For spacecraft in a medium Earth orbit (MEO), who cannot be de-orbited nor have a prescribed graveyard orbit, a different set of requirements exist as explained in Table 3.1.

Where semi-synchronous is an orbit which has a period of half a day, more specifically here it is a circular orbit with a altitude of about $22000 \mathrm{~km}$. Trans-semisynchronous is an orbit which crosses $22000 \mathrm{~km}$, this can by any highly elliptical orbit such as a geosynchronous transfer orbit (GTO) which does not cross into LEO or a Molniya orbit.

Table 3.1: End of life requirements for spacecraft in MEO [26].

\begin{tabular}{|l|l|l|}
\hline Orbit Regime & Minimum Perigee Altitude & Maximum Apogee Altitude \\
\hline Below semi-synchronous & $2000 \mathrm{~km}$ & $19700 \mathrm{~km}$ \\
\hline Above semi-synchronous & $20700 \mathrm{~km}$ & $35300 \mathrm{~km}$ \\
\hline Trans-semi-synchronous & $2000 \mathrm{~km}$ & $35300 \mathrm{~km}$ \\
\hline
\end{tabular}


Given a spacecraft's area to mass ratio as well and solar conditions estimations of de-orbit time can be approximated by Figure 3.1. Several different methods to achieve disposal are discussed here with differing levels of success and technological readiness.

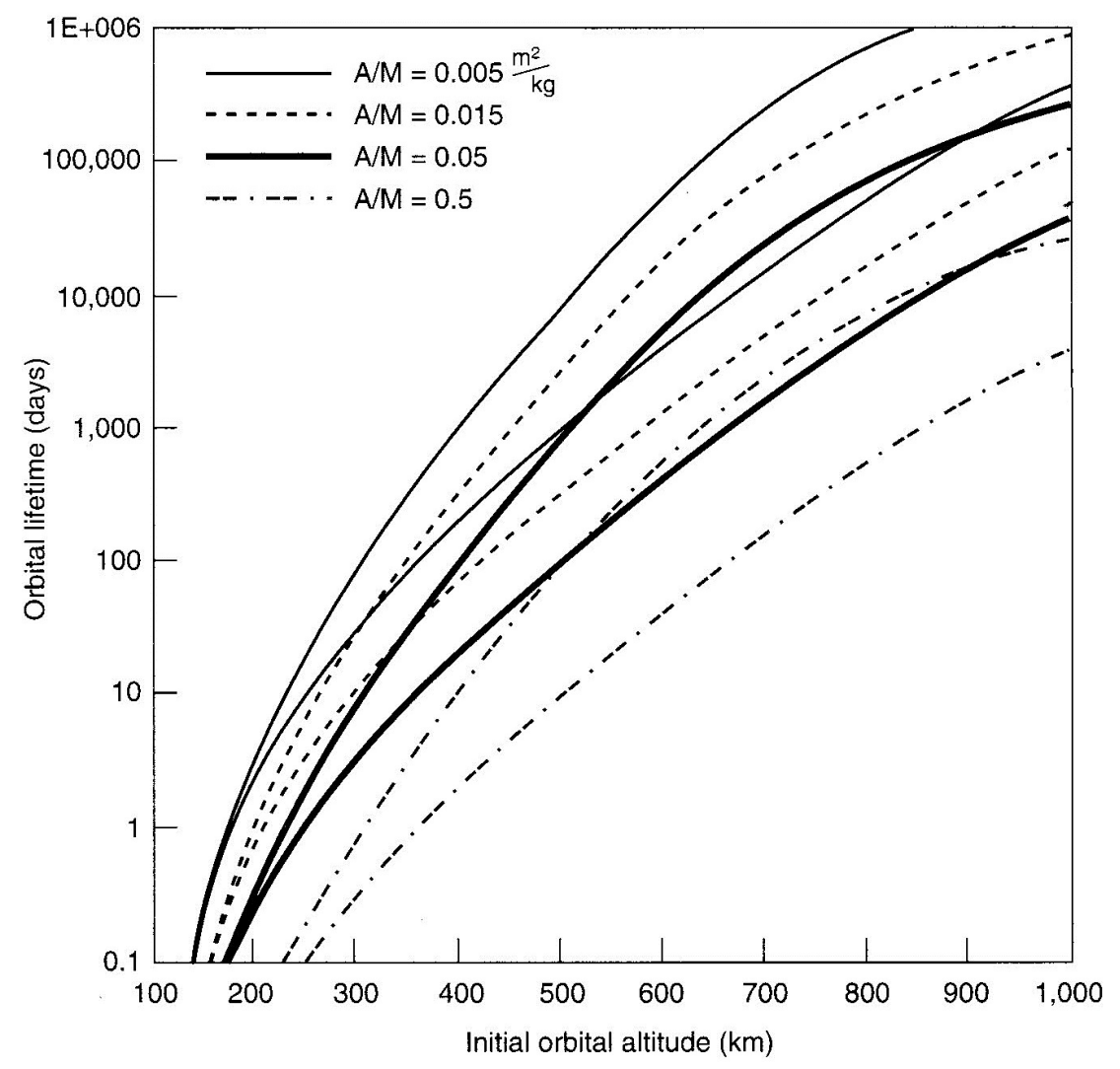

Figure 3.1: Relationship between area to mass ratio, altitude, and deorbit time [27]. Note: Two lines exist for each area to mass ratio, the upper line is for a solar minimum $(\mathrm{F} 10.7=75)$ and the lower is for a solar maximum $(\mathrm{F} 10.7=175)$.

\subsection{Natural Decay}

The simplest method to de-orbit a spacecraft is to allow it is to allow drag and solar radiation pressure to gradually lower its altitude. Some spacecraft require no additional effort to de-orbit within 25 years, this is a function of their area 
to mass ratio, initial orbit, and atmospheric conditions. NASA has set forth a simple relationship between apogee, perigee, and area to mass ratio for deorbiting within 25 years. The contour plot, Figure 3.2, assumes "mean" solar conditions and ignores solar radiation pressure.

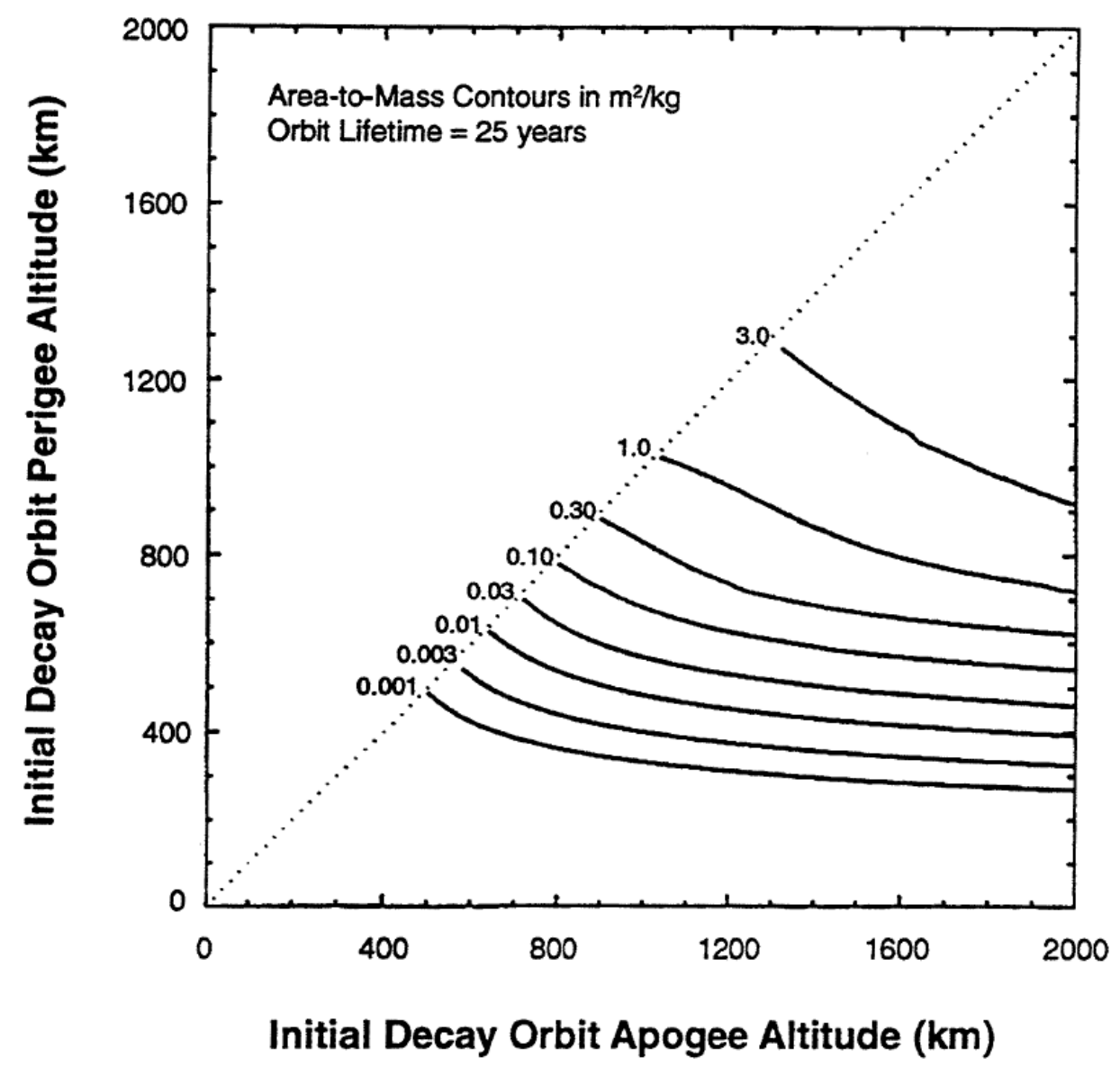

Figure 3.2: NASA's reference to area to mass ratio to de-orbit within 25 years, given apogee and perigee [6].

\subsection{Propulsion}

The fastest and most flight-proven, and arguably most expensive, option to deorbit spacecraft is LEO is by the use of a propulsive maneuver. For spacecraft 
thank are not in a LEO orbit an engine burn is often the only choice for compliance, given the requirements for its orbital regime. For example, NASA's pair of lunar orbiters, the Gravity Recovery and Interior Laboratory (GRAIL), were de-orbited and crashed into the Moon after the end of their mission on December 17, 2012 [3]. At the time of design there was no end of life disposal requirement for spacecraft outside a geocentric orbit, but were nonetheless disposed of at the end of their mission with accordance with newer guidelines. For spacecraft in GEO it is required that they are placed in a graveyard orbit $300 \mathrm{~km}$ above GEO. To accomplish this as an impulsive maneuver costs about $11 \mathrm{~m} / \mathrm{s}$ of delta-v. For example, the spacecraft GOES 12, a weather satellite, was decommissioned in August of 2013 and has been placed into a slightly eccentric graveyard orbit approximately 305-340 km above GEO [28].

Satellites in LEO have three options when it comes to de-orbiting via a propulsion burn: targeted reentry, immediate reentry, and perigee-lowering, as seen in Figure 3.3. Targeted reentry typically is necessary for manned or otherwise recov-

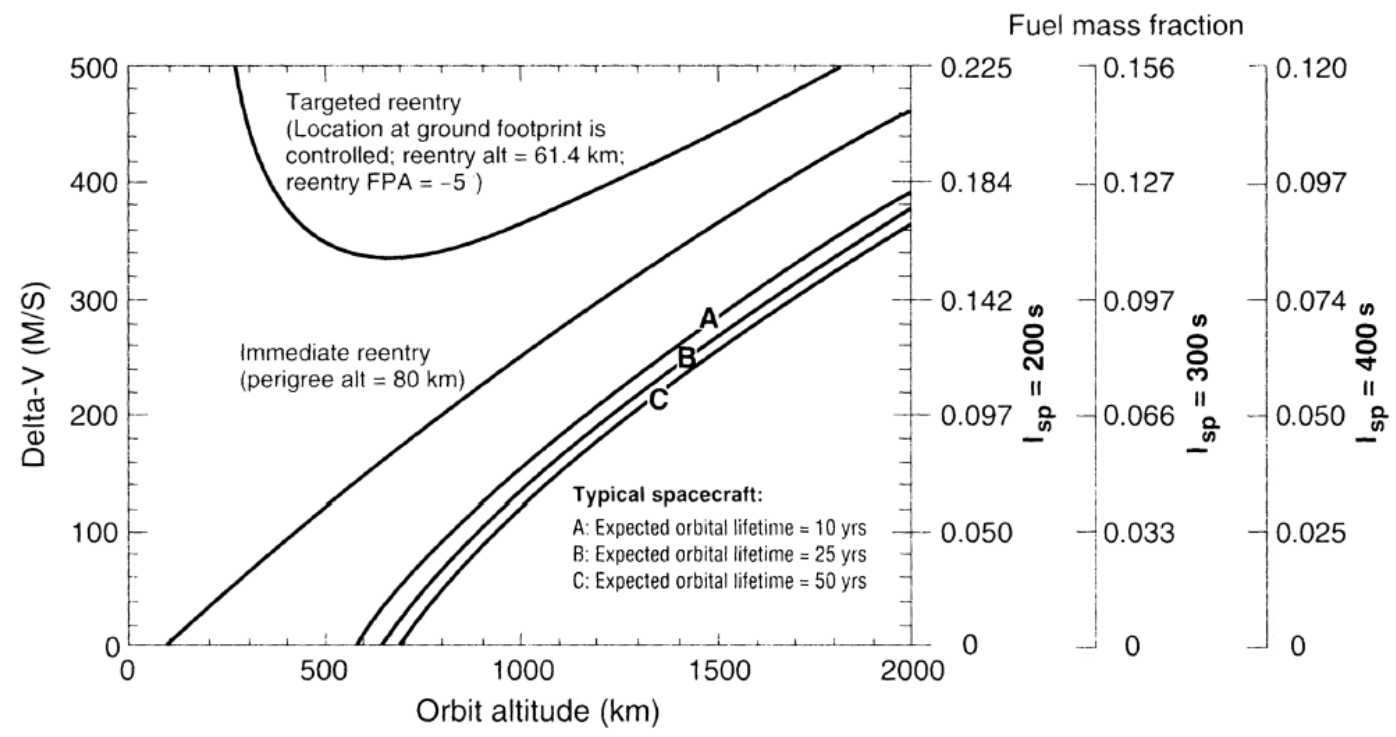

Figure 3.3: Estimated delta-v and fuel to perform a reentry burn [27]. 
erable spacecraft that must land within a given area for safe recovery, this is the most expensive option fuel-wise. Although extremely helpful to the orbital debris environment targeted and immediate reentry burns are more expensive than perigee-lowering burns. Figure 3.3 also (on the right-hand y-axes) displays the fraction of the spacecraft's mass that would be required to perform the indented de-orbit burn. For example a spacecraft at $800 \mathrm{~km}$ with an engine with an Isp of $300 \mathrm{~s}$ would require about $7 \%$ and $2 \%$ propellant mass fraction for an immediate and 25 year de-orbit time burn, respectively. Recently the spacecraft Landsat 5 was decommissioned and preformed a series of altitude lowering burns. Initially the spacecraft preformed multi-impulse maneuver to lower its altitude to lower than $700 \mathrm{~km}$ to avoid interfering with other Earth-observing spacecraft at that altitude. Then perigee-lowering burns were conducted bringing the spacecraft's expected lifetime to under 25 years [28]. This spacecraft' recent altitude history can be seen in Figure 3.4.

For spacecraft that do not already plan to have a propulsion subsystem this option is most likely prohibitively expensive. Even for a spacecraft with a propulsion system the fuel mass fraction shown in Figure 3.3, as suggested by its name, only represents the fuel mass and additional mass for additional structure and fuel $\operatorname{tank}(\mathrm{s})$ would be necessary.

\subsection{Drag Tether}

The drag tether, like the devices which will be discussed later, make use of drag to slow the spacecraft down, decreasing its orbital energy, and lowering its altitude. For a drag-only purposed tether, rather than an electromagnetic tether (see Section 3.4) the tether itself can be very lightweight although a counterweight 


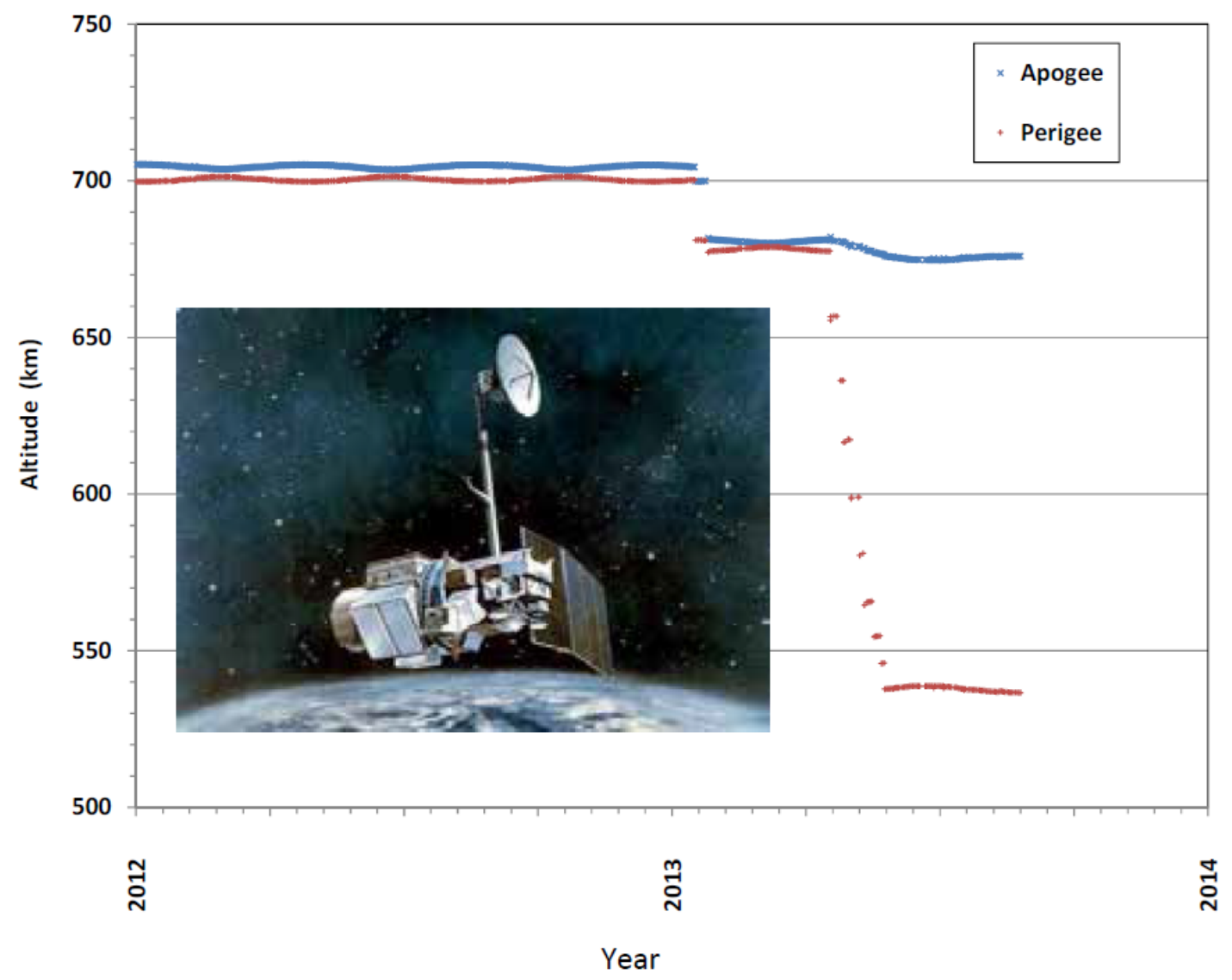

Figure 3.4: Recent altitude history for Landsat 5 [28].

placed at the end of the line is used [29]. The tether is kept in position by gravity gradient forces and is on the Earth-facing side of the spacecraft.

Although some spacecraft have successfully flown with tethers, such as the TiPS which survived many years, the SEDS 2 experiment failed several days after deployment [30]. Such a large difference between failure times for these tether mechanism begs the question, why? Was the failure of SEDS 2 an anomalous event, or is such a system too prone to failure with the current orbital debris environment? Certainty tethers, being made of thin and presumably relativity impact-vulnerable material, are susceptible to complete failure due to orbital debris/micrometeorite strike since a large enough impact placed anywhere along 
the length of the tether would sever the counterbalance and render the system useless.

\subsection{Electromagnetic Tether}

The electromagnetic tether is very similar to the drag tether, except that this system also relies on the use of magnetic effects to increase the rate of de-orbiting. As a charged object moves through a magnetic or electric field with solve velocity it experiences a force, discussed more in Section 2.4. Proposed systems of this type typically make use of a weighted end device and a current-carrying tether [29], a sample diagram can be seen in Figure 3.5.

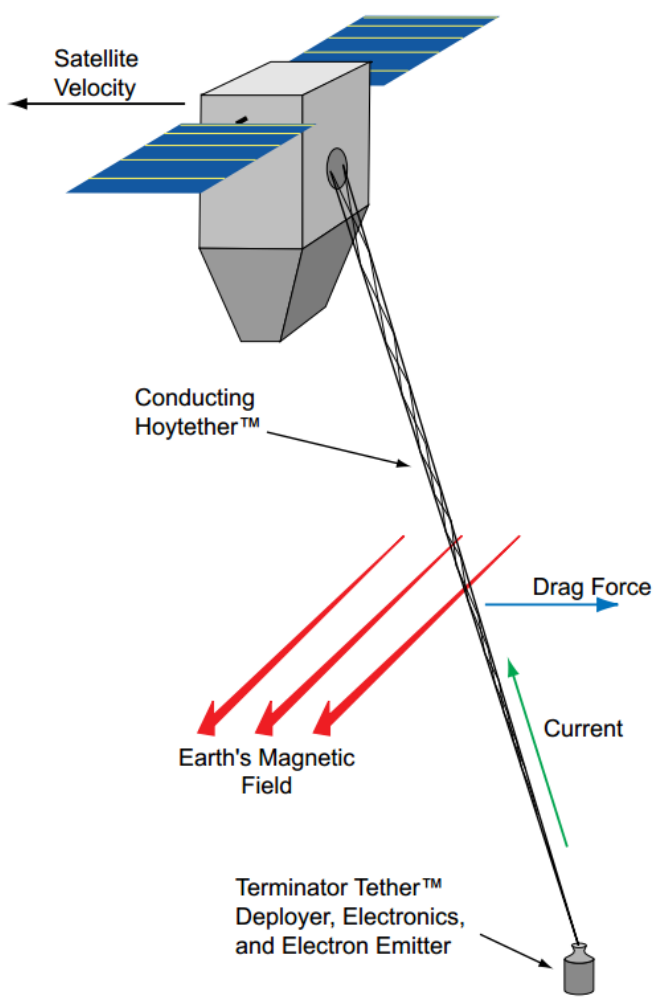

Figure 3.5: Concept of an electromagnetic tether system [31]. 
Since this device is dependent on Earth's magnetic field, its de-orbiting efficiency is dependent on its orbit. The strength of Earth's magnetic field is approximately proportional to the cosine of the inclination of the position of interest, therefore the effectiveness of the device is diminished at high inclinations [31]. For example, a $1500 \mathrm{~kg}$ spacecraft with a $30 \mathrm{~kg}$ electromagnetic tether system and $15 \mathrm{~km}$ long tether is subject to decay rates as presented in Figure 3.6. Since $44 \%$ of all spacecraft that are in LEO are in a sun-synchronous orbit (at an inclination of 98 degrees) [32] an effectiveness breakdown at such a popular orbit is unfortunate considering is efficiency at lower inclination orbits.

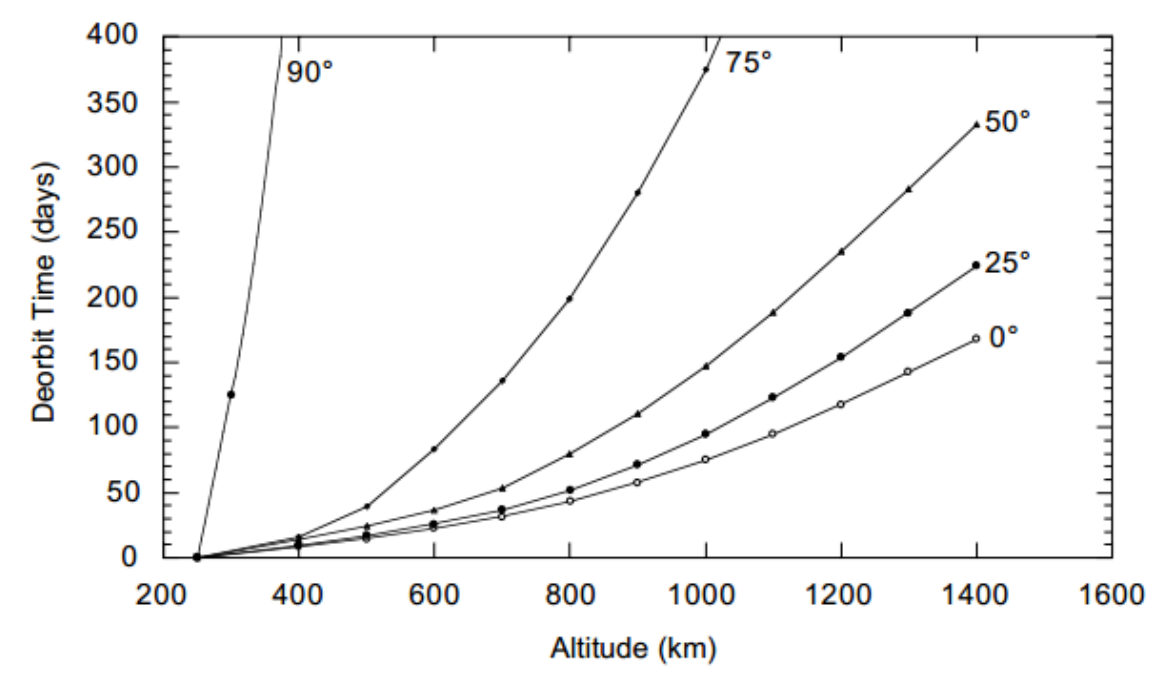

Figure 3.6: Electromagnetic tether orbital decay rates based on inclination [31].

\section{$3.5 \quad 2-D$ Sail}

A flat sail can be for the purpose to de-orbit a spacecraft by use of additional deployed area for increased drag and solar radiation pressure is a method which has some amount of flight heritage. As shown in Figure 3.1, the increase in area to mass ratio greatly effects the orbital lifetime of a body in LEO. 
California Polytechnic State University launched CP5, a 1U CubeSat, in September 2012 with the intention of deploying a de-orbiting 2-D sail [33]. Unfortunately, communication with CP5 was lost and deployment did not take place. On the 20th of January 2011 NASA deployed a $10 \mathrm{~m}^{2}$ sail aboard a $3 \mathrm{U}$ CubeSat, NanoSail-D2 [34]. The spacecraft was placed in a $641 \times 652 \mathrm{~km}$ orbit [35] with an expected orbital lifetime of 70-120 days given its $2.5 \mathrm{~m}^{2} / \mathrm{kg}$ area to mass ratio. In reality the spacecraft de-orbited in 240 days [36].

The main criticism with with 2-D devices is the need for attitude control. Results from an attitude simulation of a $140 \mathrm{~kg}$ spacecraft with a $25 \mathrm{~m}^{2}$ sail at $620 \mathrm{~km}$ and $450 \mathrm{~km}$ can be seen in Figure 3.7. Ith can be seen that at high altitudes there is no real prominent torque on a spacecraft such as this and the spacecraft seemingly tumbles randomly. It is only at low enough altitudes do aerodynamic torques become most prominent and are able to correctly orientate the spacecraft properly. NanoSail-D2 had permanent magnets as a method to passively control the spacecraft's attitude [38], which was not a powerful enough mechanism to control the spacecraft. It seems that for a spacecraft to de-orbit
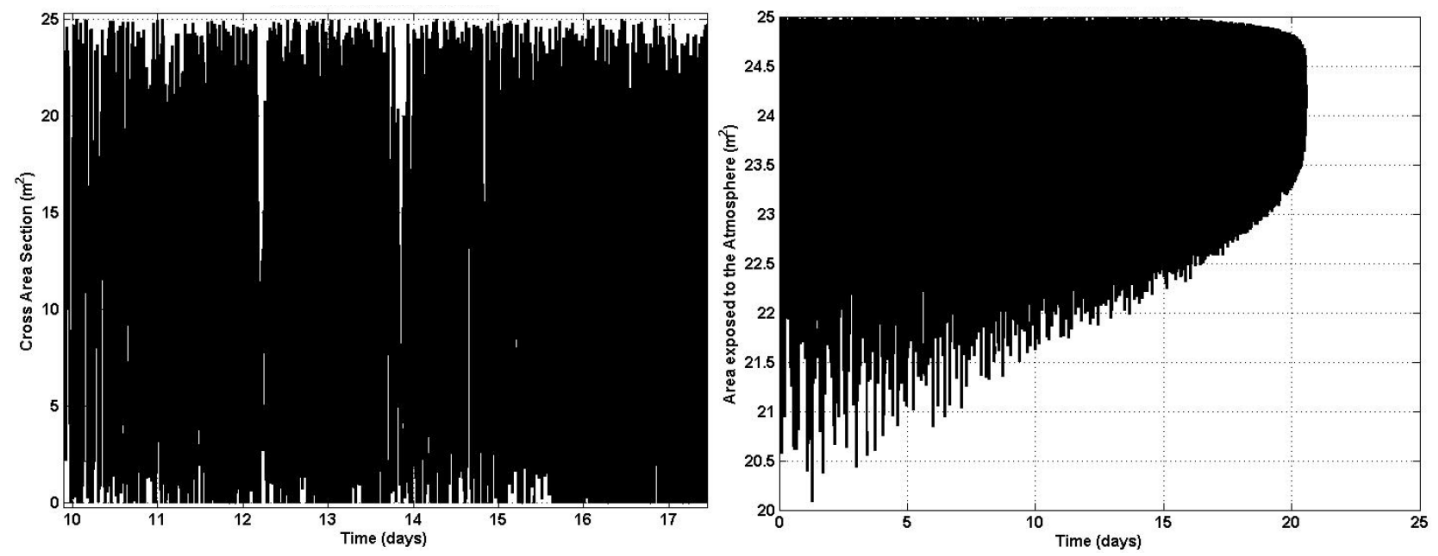

Figure 3.7: Simulated cross sectional area over time at $620 \mathrm{~km}$ (left) and $450 \mathrm{~km}$ (right) of a 2-D body in orbit [37]. Note the y-axis scale is different on each plot. 
properly with a 2-D sail it is necessary for active control.

\subsection{3-D Device}

To circumnavigate the attitude control problem presented by a 2-D sail a 3-D device presents an increased (if not constant) area at all orientations. Proposed ideas for 3-D devices are almost always require some kind of inflation mechanism. Within the topic of inflatables three types of devices exist: rigid structuresupported, fully rigid, and inflation-maintained.

All three types of inflatables make use of a thin-film (gossamer) body, on which drag and solar radiation pressure will act, and inflating gas. The amount of gas necessary is dependent on the external pressure experienced by the body, the volume that must be inflated, and temperature. From here the three different systems begin to differ. The structure-supported body makes use of internal rigid "piping" to maintain the desired shape, as seen in Figure 3.8. The internal structure is supported by rigidizing coating which is either thermally activated or cured by UV light. The fully rigid body has no internal structure and is completely coated in a coating. Implications for a large rigid structure are mainly the risk of fragmentation due to orbital debris or micrometeorite strike. The inflation-maintained design relies on a nearly constant supply of gas to preserve the proper level of inflation.

The differences between the structurally-supported device and the fully rigid device are relativity minor when compared to the implications of an inflation maintained device. The inflation-maintained option requires much more gas than either of the rigidizing options, but what is more of a design concern is that it is not a passive system. A system of power supply, distribution, computing, valves, 


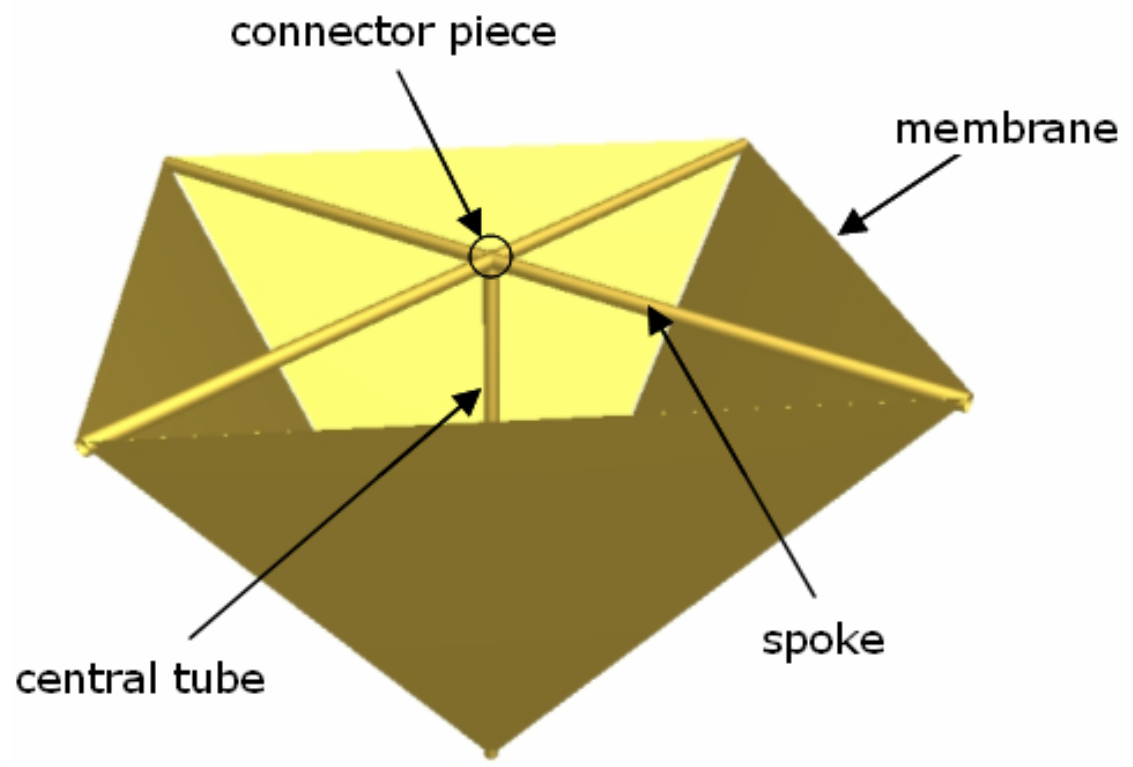

Figure 3.8: Diagram showing the components of a structurallysupported 3-D device [39].

and communication and/or pressure monitoring equipment must be operational during the life of the de-orbiting maneuver. The importance of these requirements are hard to overstate since the spacecraft bearing the device would be at end of life and may be experiencing failing components.

The Global Aerospace Corporation has proposed an inflation-maintained spherical de-orbit device, rendering seen in Figure 3.9. It has been shown the a $37 \mathrm{~m}$ diameter sphere attached to a $1200 \mathrm{~kg}$ spacecraft at a $833 \mathrm{~km}$ sun-synchronous orbit would successfully de-orbit the spacecraft in one year while requiring less than $1 \mathrm{~kg}$ of gas to maintain proper internal pressure despite orbital debris and micrometeorite impacts [40][41]. 


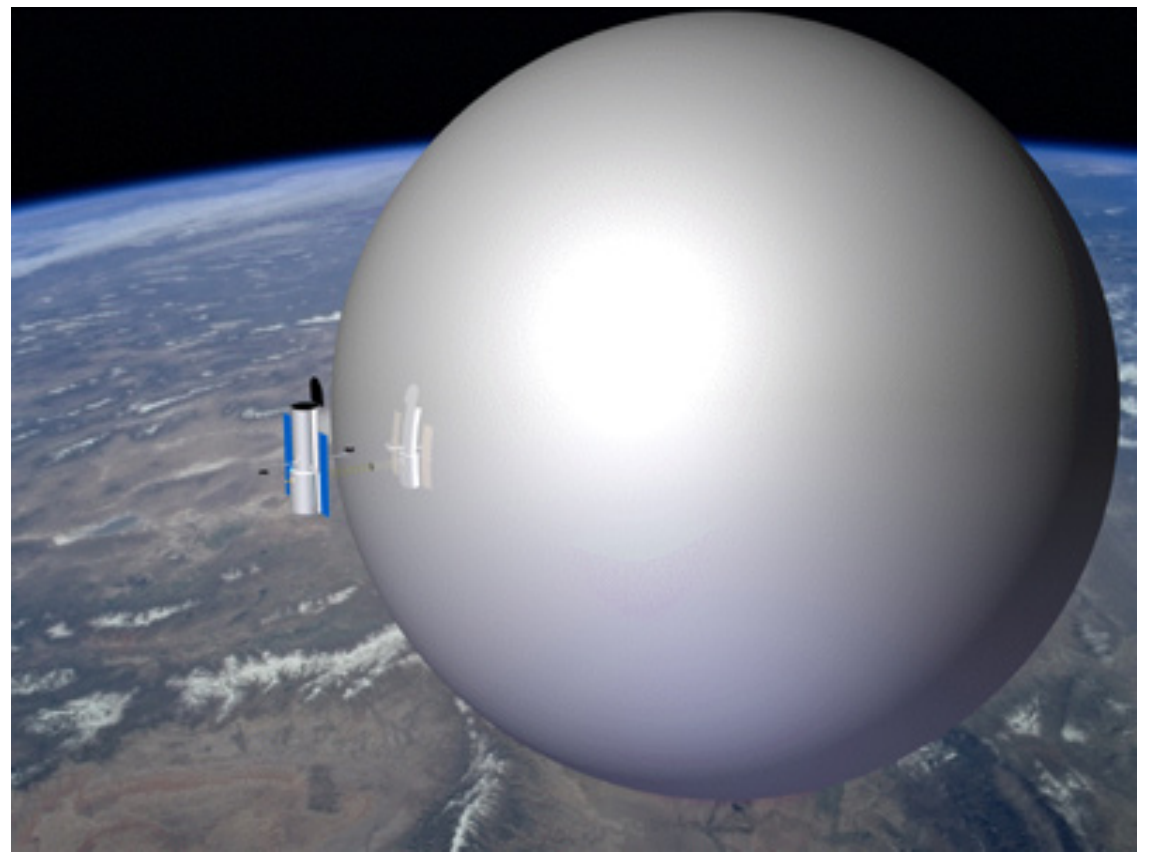

Figure 3.9: Rendering of a spacecraft with an attached inflatable device [40]. 


\section{Analysis}

As mentioned previously, a major concern with a structure whose shape is maintained purely by a high pressure interior is the amount of gas that will be leaked out into the environment. The leak analysis will be the primary topic of discussion in this chapter, the reader is encouraged to go to Chapter 2 for a review of orbital dynamics.

The process presented in the flowchart in Figure 4.1 is the algorithm used to calculate the leak rate from orbital debris and micrometeorite impacts. The algorithm is broken down into four areas of calculation: orbital debris and micrometeorite flux, hypervelocity impacts, leak rate, and inflation. Each area will be discussed individually here and results will be discussed in Section 5.2.

\subsection{Orbital Debris and Micrometeorite Flux}

Much work has been done in order to characterize the current and near-future orbital debris environment in order assess the risk to spacecraft. Information about the orbital debris environment that a spacecraft is likely to experience can be extremely helpful to engineers. In this case, predictions of likely orbital debris an micrometeorite (MM/OD) strikes help estimate the leak rate out of the inflatable envelope. 


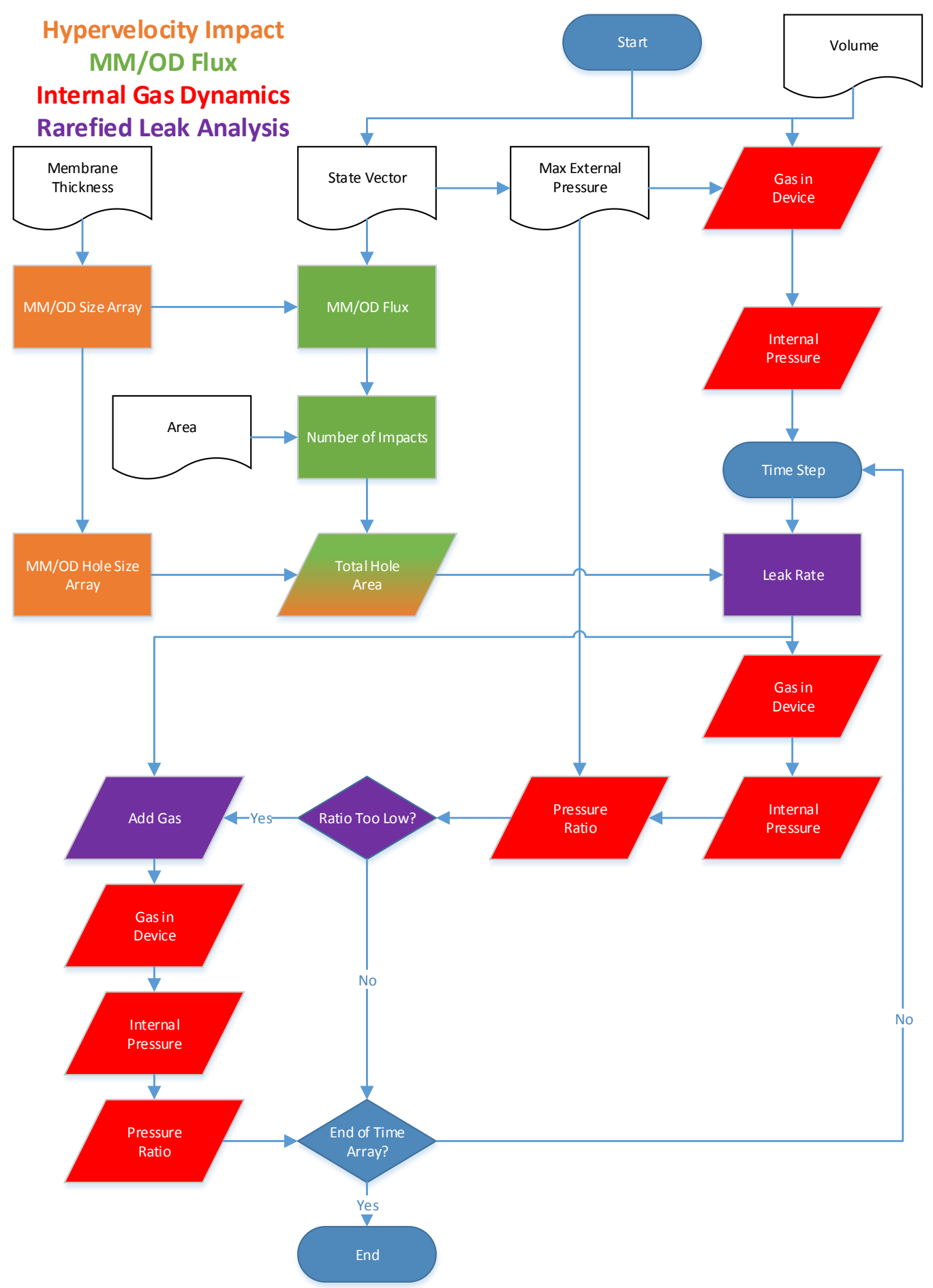

Figure 4.1: Analysis flow chat. 


\subsubsection{Orbital Debris Flux}

For in-depth analysis of a spacecraft's risk to orbital debris impact, NASA's Orbital Debris Program Office has developed the Orbital Debris Engineering Model, ORDEM2010. The program takes in certain orbit parameters as well as the year of interest, and outputs orbital debris flux values for six different size ranges, $10 \mu \mathrm{m}, 100 \mu \mathrm{m}, 1 \mathrm{~mm}, 1 \mathrm{~cm}, 10 \mathrm{~cm}$, and $1 \mathrm{~m}$ [42].

In 1989 Kessler, Reynolds, and Anz-Meador developed a simple analytical model to calculate orbital debris flux given user parameters [43], known as the Kessler model. Unforeseen to the team was growth of the orbital debris population. In 1991 adjustments were made to the model to match the current orbital debris environment [44], known as the revised Kessler model. The model outputs a flux value, $F$, based on a given debris size and larger, and is a function of altitude, inclination, year, and solar activity.

$$
F=H \times \Phi \times \Psi \times\left(F_{1} \times g_{1}+F_{2} \times g_{2}\right)
$$

The term $\Psi$ is a function of the inclination, and is given by a table that can be found in Table A.2. The following equations are used to calculate the necessary values:

$$
\begin{gathered}
H=\sqrt{10 \exp ^{-\frac{(\log (d)-0.78)^{2}}{0.637^{2}}}} \\
\Phi_{1}=10\left(\frac{h}{200}-\frac{S}{140}-1.5\right)
\end{gathered}
$$




$$
\Phi=\frac{\Phi_{1}}{\Phi_{1}+1}
$$

If the year in question is before 2011 Equation 4.5 is used, else Equation 4.6.

$$
\begin{gathered}
g_{1}=(1+q)^{(t-1988)} \\
g_{1}=(1+q)^{23}\left(1+q^{\prime}\right)^{(t-2011)} \\
g_{2}=1+p(t-1988) \\
F_{1}=1.22 \times 10^{-5} d^{-2.5} \\
F_{2}=8.1 \times 10^{10}(d+700)^{-6}
\end{gathered}
$$

where $d$ is the diameter of the orbital debris (and larger) desired, $t$ is the year, $S$ is the 13 month smoothed solar flux (F10.7) value, $q$ and $q$ ' are the assumed annual growth rate of orbital debris fragments in orbit, and $q$ is the assume annual growth rate of total mass in orbit. It will be assumed that $q, q^{\prime}$, and $q$ are 0.02 , 0.04 , and 0.05 , respectively. An example plot using this function can be seen in Figure 4.2. 


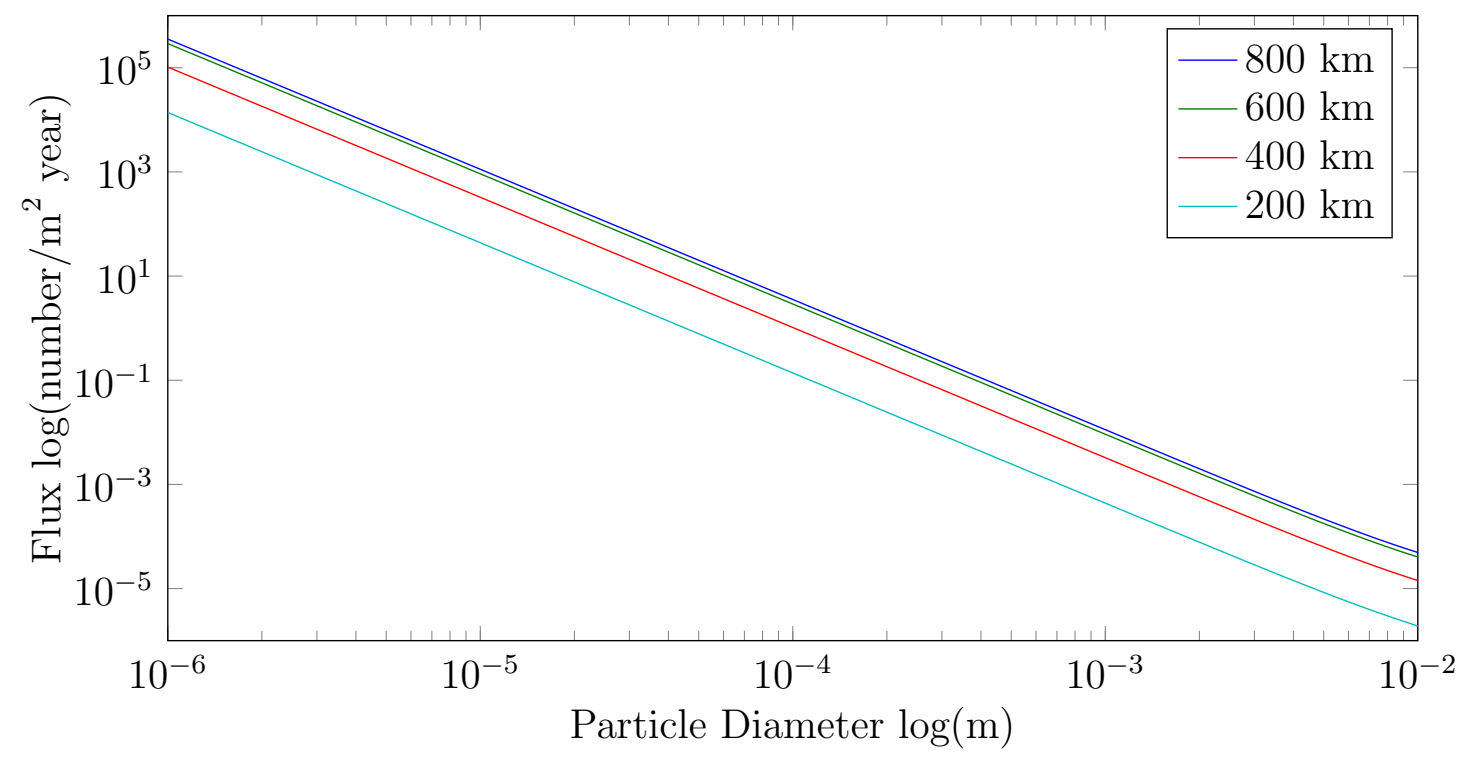

Figure 4.2: Orbital debris flux of objects $1 \mu \mathrm{m}$ to $1 \mathrm{~cm}$, given a sunsynchronous orbit in 2013 , at various altitudes.

\subsubsection{Micrometeorite Flux}

The micrometeorite flux, although less than the current orbital debris flux, is still very dangerous because the average spacecraft/micrometeorite impact speed is many kilometers per second faster than the average spacecraft/orbital debris impact [13]. The following algorithm is used to calculate the micrometeorite flux is and is only dependent on altitude. It will be assumed for this project that the micrometeorite is not dependent on spacecraft orientation. Further, the algorithm [13], is a function of micrometeorite mass, and not diameter, as the orbital debris flux is. To relate the mass to the diameter Table 4.1 will assume densities based on diameter. 
Table 4.1: Assumed densities of micrometeorites based on diameter $[13]$.

\begin{tabular}{l|l}
\hline Diameter Range $(\mathrm{cm})$ & Density $\left(\mathrm{g} / \mathrm{cm}^{3}\right)$ \\
\hline $0-0.01$ & 2.0 \\
$0.01-0.27$ & 1.0 \\
$0.27+$ & 0.5 \\
\hline
\end{tabular}

For the micrometeorite flux, let:

$$
\begin{gathered}
X=\frac{R_{\oplus}+100}{R_{\oplus}+h} \\
\eta=\arcsin (X) \\
A=15+2.2 \times 10^{3} m^{0.306} \\
B=1.3 \times 10^{-9}\left(m+m^{2} \times 10^{11}+m^{4} \times 10^{27}\right)^{-0.306} \\
C=1.3 \times 10^{-16}\left(m+m^{2} \times 10^{6}\right)^{-0.85}
\end{gathered}
$$

where $R_{\oplus}$ is the radius of Earth, $\mathrm{h}$ is the altitude, and $\mathrm{m}$ is the mass of the micrometeorite in grams.

$$
\begin{gathered}
F_{M M}=3.156 \times 10^{7}\left(A^{-4.38}+B+C\right) \\
F_{\text {grav }}=1+X
\end{gathered}
$$




$$
F_{\text {shield }}=\frac{1+\cos (\eta)}{2}
$$

Finally,

$$
F=F_{M M} \times F_{\text {grav }} \times F_{\text {shield }}
$$

Figure 4.3 shows the micrometeorite flux at $800 \mathrm{~km}$, note the spikes at about $0.1 \mathrm{~mm}$ and $2.5 \mathrm{~mm}$. These jumps in the function are due to the density assumptions listed in Table 4.1.

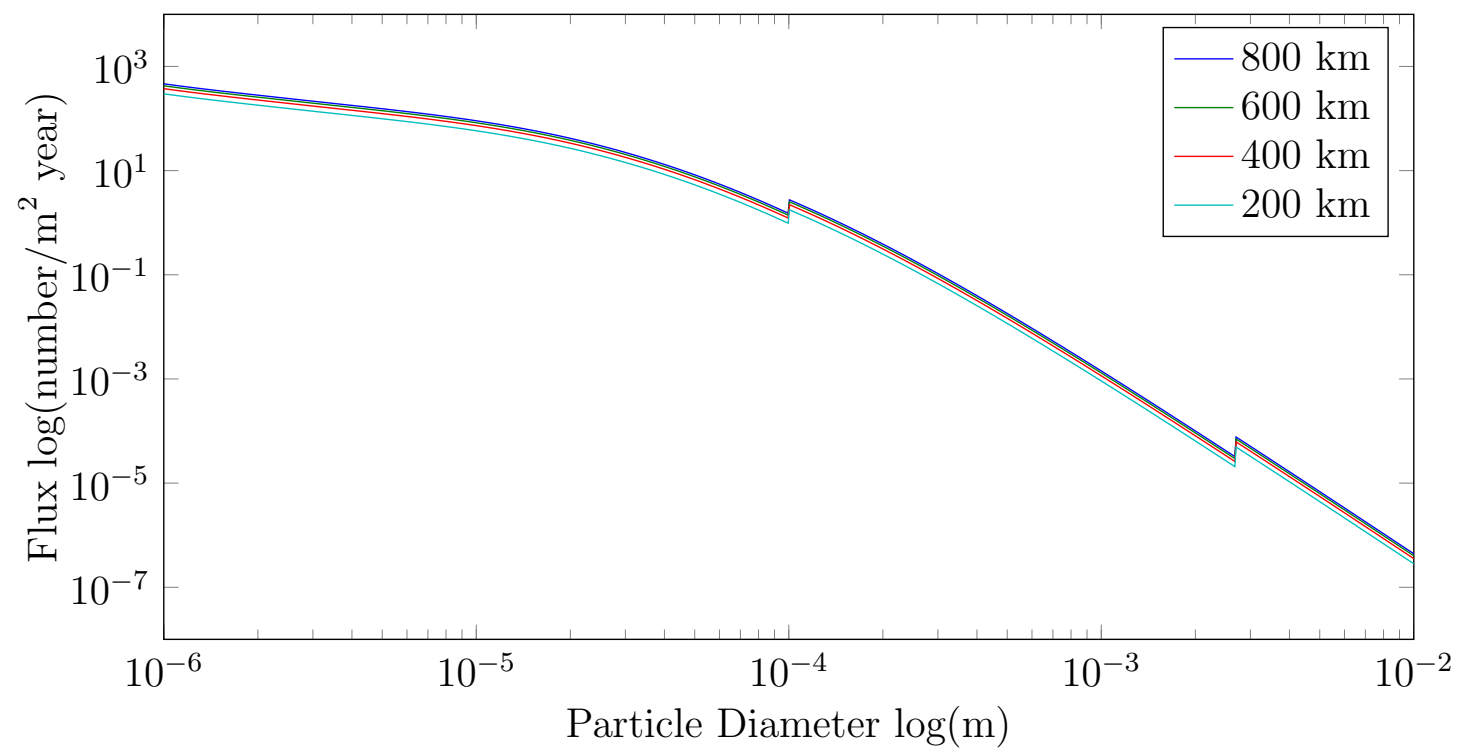

Figure 4.3: Micrometeorite flux of objects $1 \mu \mathrm{m}$ to $1 \mathrm{~cm}$, at various altitudes

\subsection{Hypervelocity Impacts}

Collisions in LEO often occur at relative speed on the order of many kilometers per second, the physics of these collisions are dominated by hypervelocity impacts. Here three main types of results can occur from an impact: cratering, near incipient penetration, and complete penetration [45], as seen in Figure 4.4. 

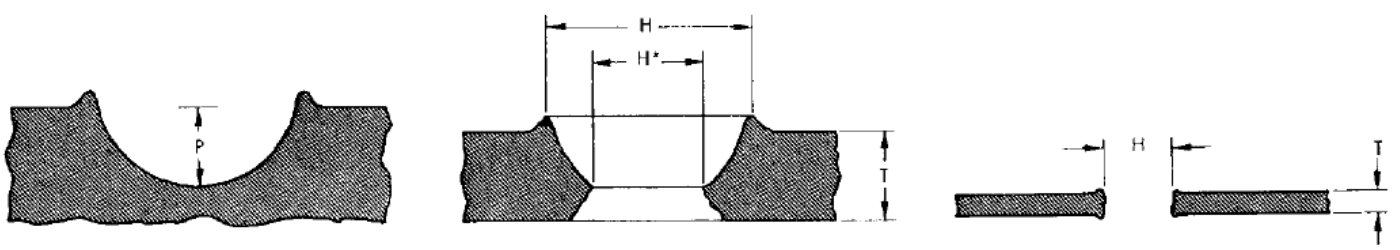

Figure 4.4: Three types of hypervelocity impacts: cratering (left), near incipient penetration (center), and complete penetration (right) [45].

Even though the membrane used in the inflatable device will be very thin, on the order of micrometers, not all MM/OD particles that strike it will penetrate the membrane. For a "thin plate" the penetration thickness, $P$, is given by:

$$
P=\frac{1}{2} d \sqrt[3]{\frac{24 \rho_{p} v^{2}}{E}}
$$

where $d$ is the particle diameter, $v$ is the impact velocity, $\rho_{p}$ is the density of the particle, and $E$, is the Young's modulus of the plate. It will be assumed that the impact speed for orbital debris is $10 \mathrm{~km} / \mathrm{s}$ [44] and $19 \mathrm{~km} / \mathrm{s}$ for micrometeorites [13]. The Young's modulus, $E$ for a thin-film material will assumed to be 1690 $\mathrm{kg} / \mathrm{m}^{2}$ [46], and density for the impacting particles is $2 \mathrm{~g} / \mathrm{cm}^{2}$ [13].

There are seven different outcomes from a hypervelocity impacts on a thinfilm target, which is mainly a function of particle size and energy, among other factor [41]. These possible outcomes are shown in Figure 4.5.

For simplification, if the incident particle is not able to fully penetrate the membrane it effects the membrane in no way. It will be assumed, both for simplicity and for sake of a conservative analysis, that any particle that penetrates the membrane does not decompose in any manner which would increase the internal pressure inside of the inflatable. In reality, small particles that make it through the film will vaporize is such a way as to help maintain internal pressure 


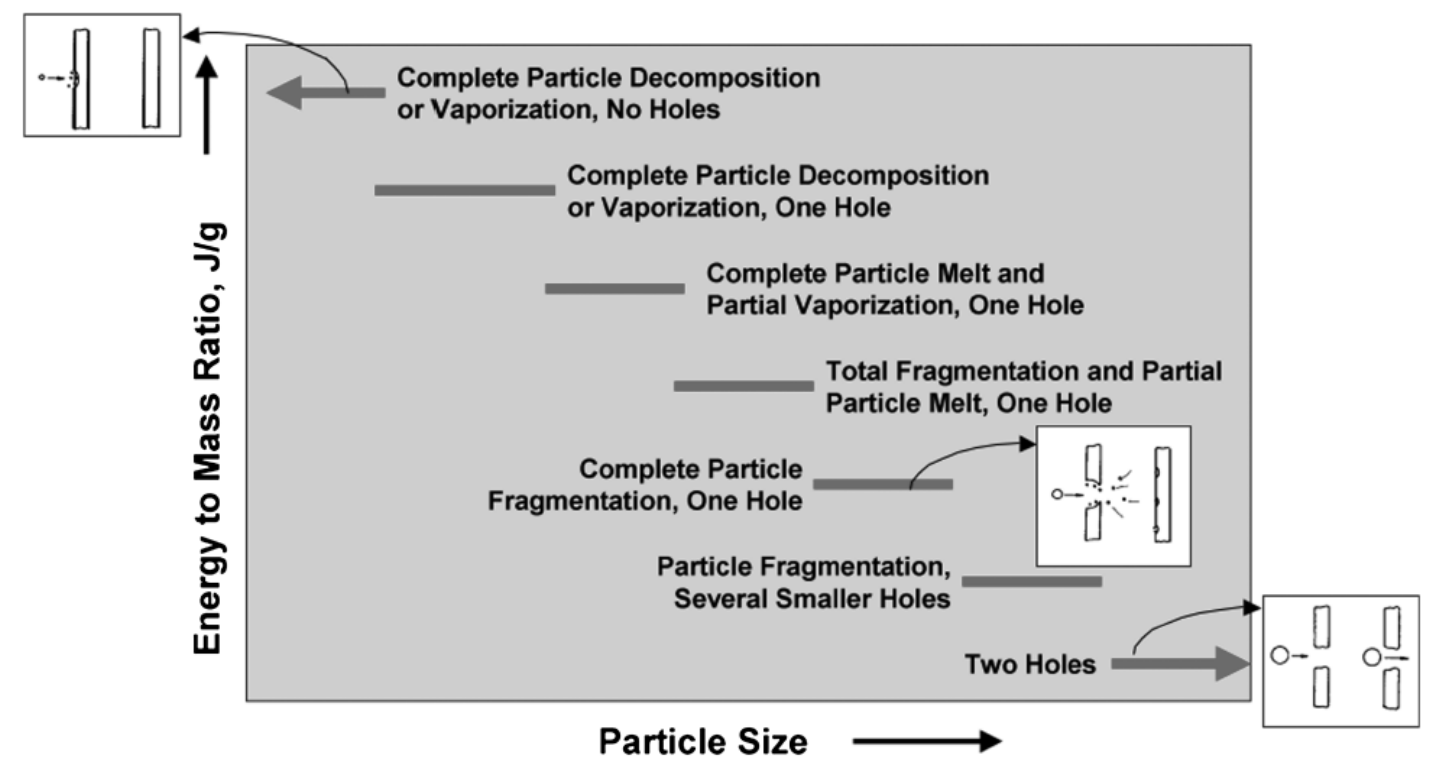

Figure 4.5: Outcomes of hypervelocity impact on thin-film material [41].

[41], this will be ignored. Furthermore, it will be assumed that a particle will completely penetrate both sides if it is able to simply penetrate twice the membrane thickness. This assumes that the particle does not decompose or fragment in a way which adversely effects its ability to penetrate the film.

The relationship between particle diameter and the resulting total hole area can be seen in Figure 4.6. Due to the assumptions in this section more particles will pass through both sides of the inflatable device than what in reality would. It can be seen that micrometeorites will penetrate the membrane at smaller diameters than the orbital debris particles. Since it is also assumed that the hole diameter, once a hole is formed, is only a function of particle diameter, the lines of hold area for micrometeorites and orbital debris are equal when the same number of layers are penetrated. The four jumps in hole area are due to the number of layers being penetrated. 


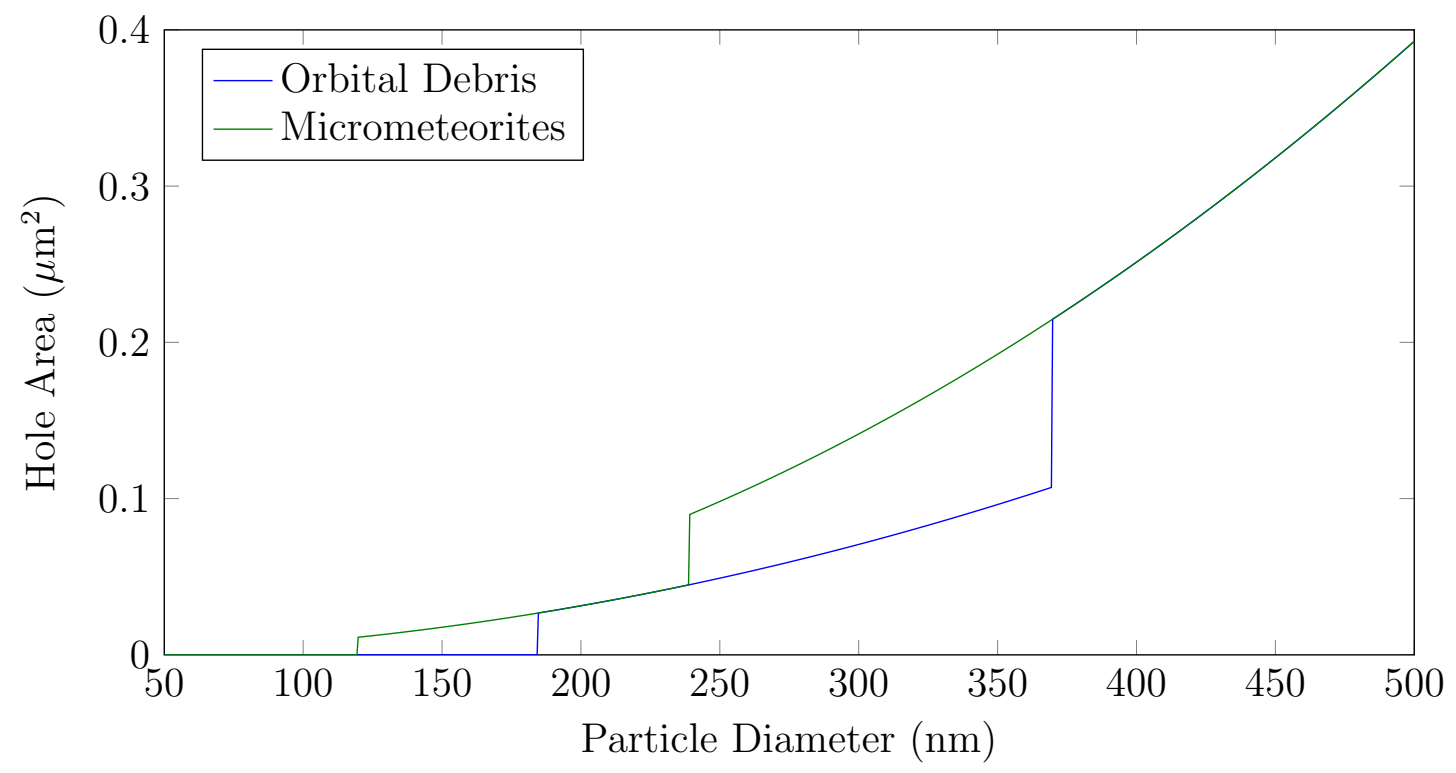

Figure 4.6: Particle diameter and resulting hole area

\subsection{Rarefied Gas Flow Through an Orifice}

Since the internal pressures inside the proposed inflatable envelope is very low the resulting mean free path inside of the device is much greater than 1 , the flow inside the device is dominated by rarefied gas flow physics. The flow of a rarefied gas through a thin orifice is given by [47]

$$
\dot{m}=\frac{\sqrt{\pi} r^{2}}{v_{m p}} P
$$

where $r$ is the radius of the orifice, $v_{m p}$ is the most probable velocity of the gas molecules (see Equation 2.19), and $P$ is the pressure of the high-pressure zone (inside the envelope). It is assumed in this model that the exit of the orifice is a perfect vacuum and there is zero mass flow rate into the system. 


\section{Results}

Before much work can be performed the orbit propagator must be validated, and it must also be determined what orbital perturbations are necessary for this project. For computational efficiency only perturbations that effect the orbital lifetime of a given spacecraft will be considered important. Analysis was first conducted using AIG's Systems Tool Kit (STK) to determine which perturbations should be included in the final orbit propagator, a custom-build program written in MATLAB.

The orbital perturbations to be tested were: non-spherical Earth (J2 and J2 through J6), drag, solar radiation pressure, n-body effects (Moon and Sun). The starting orbit was an $800 \mathrm{~km}$ circular orbit. The spacecraft was fixed with an area to mass ratio of 2.4154, a coefficient of drag of 2.2 , and a coefficient of reflectivity of 1.8. The atmospheric model used was the U.S. Standard Atmosphere 1976. Results from this analysis, with percent error (as compared with Case H) can be seen in Table 5.1. Case $\mathrm{H}$ will be considered to be the true orbit, this is an incorrect statement but will assumed because its propagation included the most perturbations.

It can be shown that with a requirement of de-orbiting within a one percent error of Case $\mathrm{H}$ that Case D sufficiently meets this with minimal computational cost. The orbit propagator was written in MATLAB using Cowell's method and the J2, drag, and solar radiation pressure perturbations. An function was created 
Table 5.1: De-orbit results using different orbital perturbations.

\begin{tabular}{l|l|l|l}
\hline Case & Perturbation(s) & De-Orbit Time & Percent Error \\
\hline A & Drag & 372.36 days & 13.491 \\
B & Drag,J2 & 335.40 days & 2.223 \\
C & Drag,SRP & 226.53 days & 30.956 \\
D & Drag,SRP,J2 & 328.12 days & 0.006 \\
E & Drag,SRP,J2,n-body(Sun) & 328.12 days & 0.006 \\
F & Drag,SRP,J2-J6 & 328.10 days & 0.001 \\
G & Drag,SRP,J2-J6,n-body(Sun) & 328.10 days & 0.001 \\
H & Drag,SRP,J2-J6,n-body(Sun,Moon) & 328.10 days & \\
\hline
\end{tabular}

to stop the propagation when the orbit reached an altitude of $100 \mathrm{~km}$, which will be considered reentry. Other settings can be seen in Table 5.2.

The orbit propagator was compared to results from STK using many of the same input parameters. Unfortunately, the author is unable to determine some of the settings within STK, or unable to match its settings without a considerable amount of effort. STK uses a Runge-Kutta 7-8 ODE solver with a $7^{\text {th }}$ order Lagrangian interpolation scheme while the orbit propagator uses a simple RungeKutta 4-5 scheme. The author was unable to determine what value of nominal solar radiation pressure $\left(\mathrm{P}_{S R}\right)$ STK is using or how it calculates when the spacecraft crosses into the umbra/penumbra. Results from the comparison of STK to orbit propagator results can be seen in Figure 5.1. The comparison presented

Table 5.2: Orbit propagator settings and parameters.

\begin{tabular}{l|l}
\hline Parameter & Setting \\
\hline Solver & ode45 \\
Absolute Tolerance & $1 \mathrm{E}-6$ \\
Relative Tolerance & $1 \mathrm{E}-6$ \\
Solar Radiation Pressure & $9.55 \mu \mathrm{P}$ \\
$\mathrm{C}_{R}$ & 1.5 \\
$\mathrm{R}_{\oplus}$ & $6378.1363 \mathrm{~km}$ \\
$\mu_{\oplus}$ & $398600.4415 \mathrm{~km}^{3} / \mathrm{s}^{2}$ \\
\hline
\end{tabular}


contained the same parameters as those in Cases A-H mentioned previously.

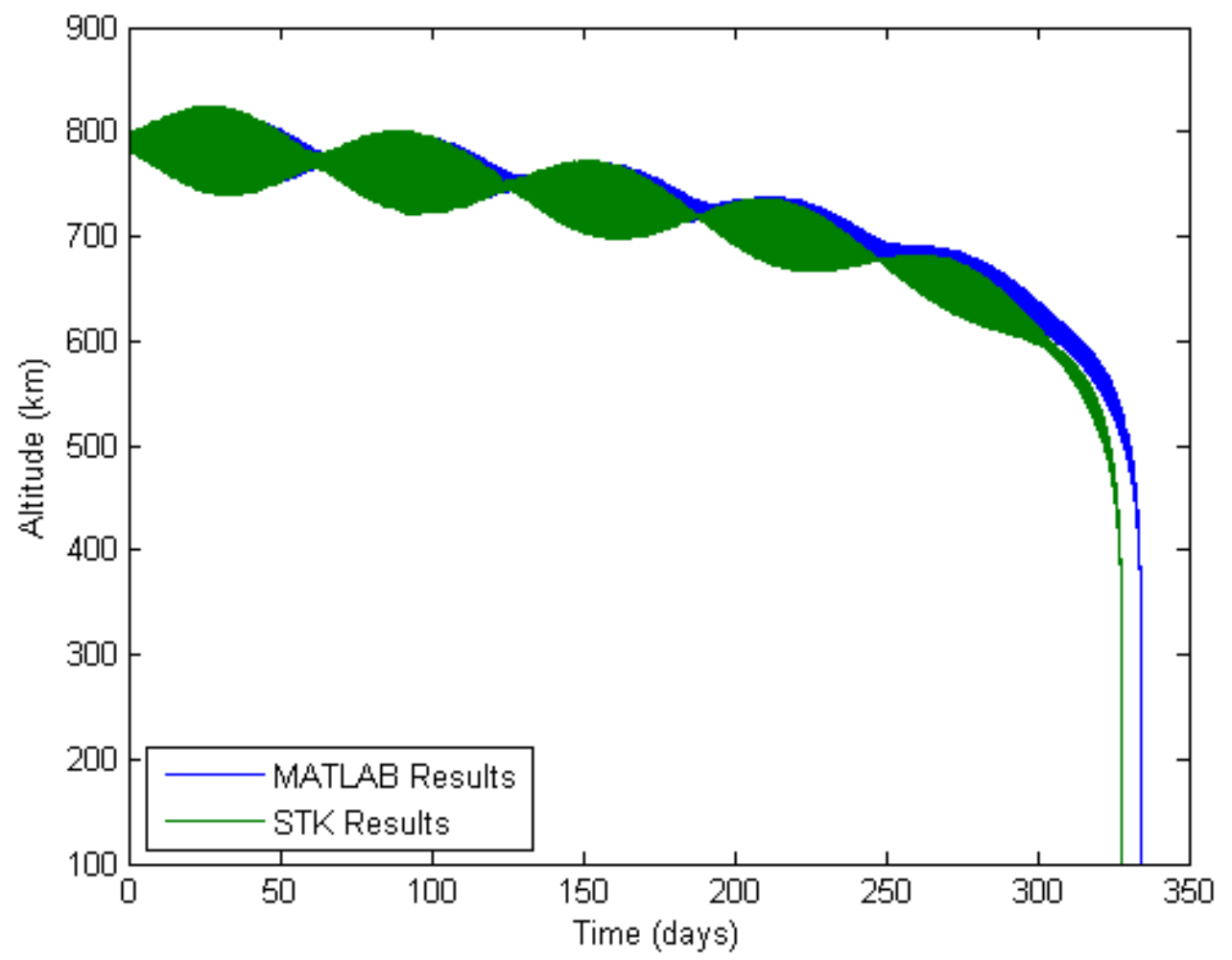

Figure 5.1: Comparison of STK and orbit propagator results from a simulation of an $800 \mathrm{~km}$ zero inclination orbit.

Results show that the spacecraft propagated with STK de-orbited in 328 day, and the spacecraft propagated with orbit propagator de-orbited in 334 days, a difference of roughly $1.9 \%$. Given the uncertainties in how STK actually propagates the orbit in time, the results from this comparison will be sufficient to call the orbit propagator accurate enough for this project. 


\subsection{System Sizing and Results}

Before system sizing can begin, a full design space must be identified. Figure 5.2 shows the de-orbit time based on starting altitude and the bodies ballistic coefficient. All orbits begin as circular and equatorial, with the same setting presented in Table 5.2.

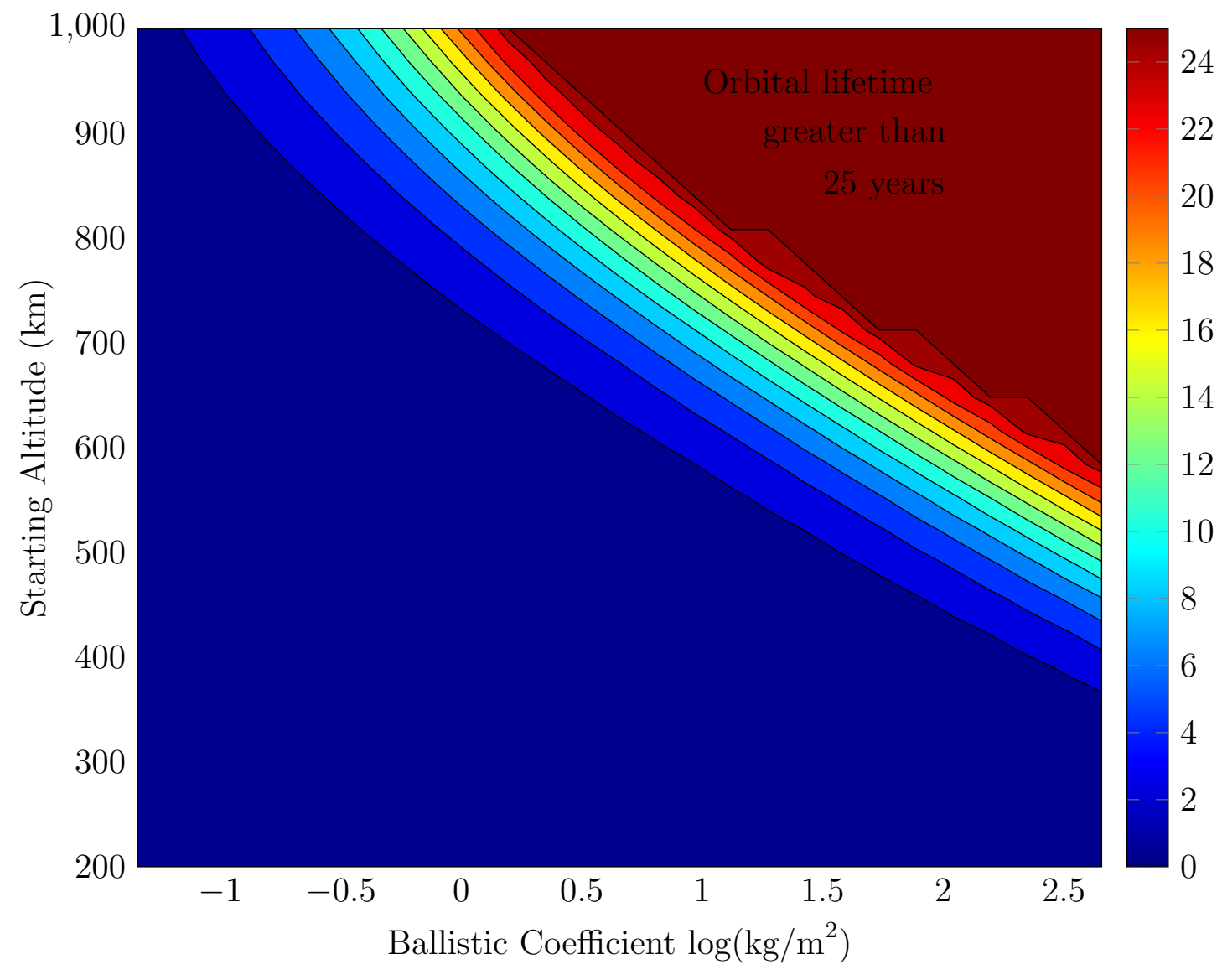

Figure 5.2: De-orbit time, in years, based on starting altitude and ballistic coefficient.

From here a hypothetical mission was devised: the spacecraft is a $1 \mathrm{U}$ CubeSat with a mass of $1.3 \mathrm{~kg}$ and begins with an $800 \mathrm{~km}$ Sun-synchronous orbit. The goal of the system will be to de-orbit the spacecraft within a year under mean solar conditions. Figure 5.2 shows de-orbit times assuming a constant coefficient 
of drag of 2.2, for here on the drag coefficient analysis that was discussed in Section 2.2 .2 will be used. In this simulation the attached inflatable sphere has a diameter ranging from $0.5 \mathrm{~m}$ to $2 \mathrm{~m}$, leading to a range of area to mass ratios of $0.2 \mathrm{~m}^{2} / \mathrm{kg}$ to $3.1 \mathrm{~m}^{2} / \mathrm{kg}$. The results can be seen in Figure 5.3. Above an area to mass ratio of $0.6 \mathrm{~m}^{2} / \mathrm{kg}$, for an initial $800 \mathrm{~km}$ orbit, the system begins to see greatly diminishing returns, especially when total system mass is taken into account. As a note, the orbital lifetime of a $1 \mathrm{U}$ CubeSate at $800 \mathrm{~km}$ without any method of PMD is on the order of 150-1500 years, shown in Figure 3.1.

Presented are the estimated mass and the estimated stored volume of the membrane based on its diameter and thickness. Both estimated mass and the

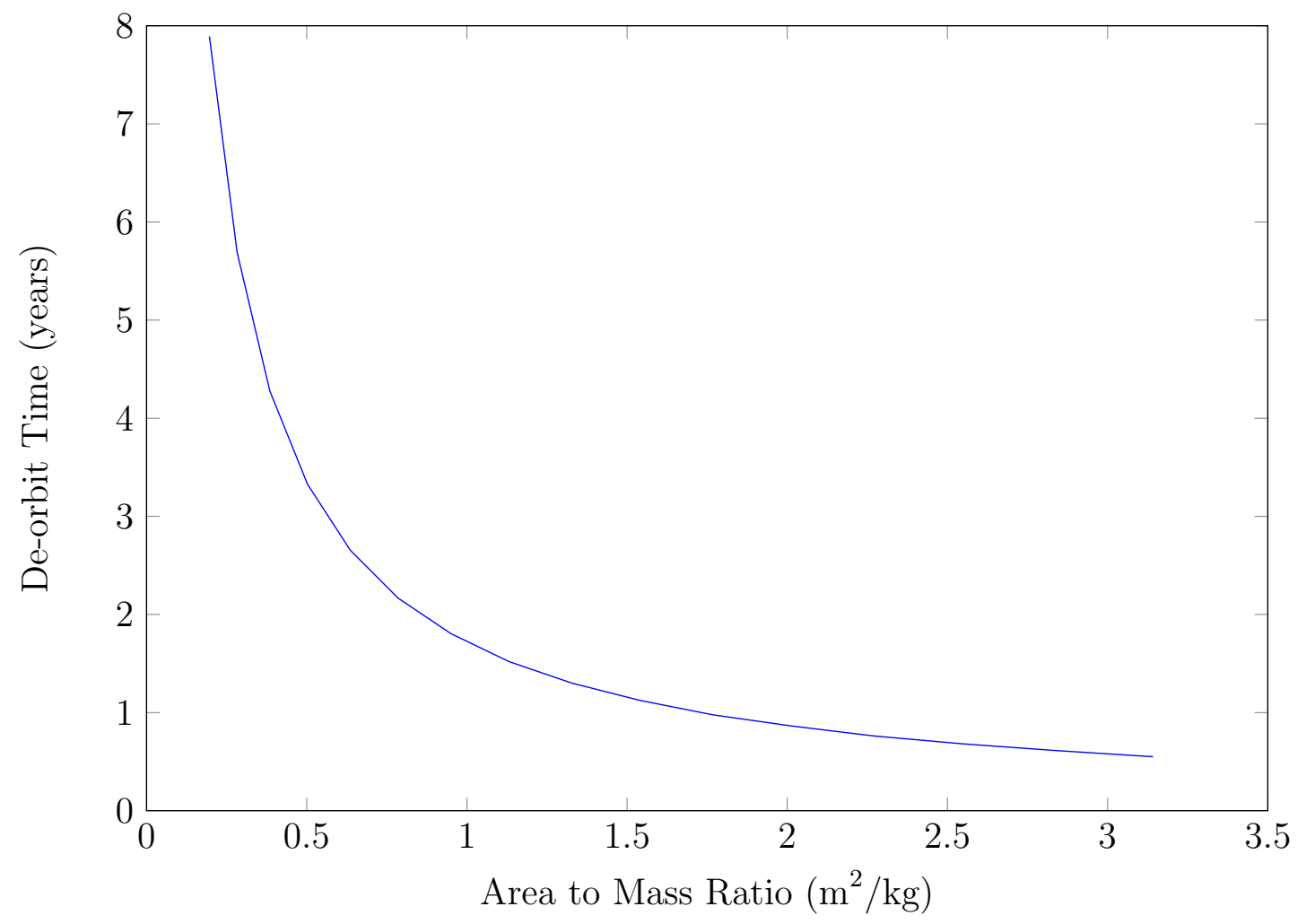

Figure 5.3: De-orbit time based on inflatable device size. 
estimated stored volume, Figure 5.4 and Figure 5.5, assume that the membrane will be assembled from pieces and includes a $4 \%$ area overlap. For storage volume is is also assumed that there is a $25 \%$ packing efficiency, that is $75 \%$ of the volume contain the stored device is empty space. The assumed densities are based on MLI thickness and can be found below in Table 5.3.

Table 5.3: Membrane area densities [46].

\begin{tabular}{l|l}
\hline Thickness $(\mu \mathrm{m})$ & Area Density $\left(\mathrm{g} / \mathrm{m}^{2}\right)$ \\
\hline 6 & 9 \\
12.5 & 17 \\
25 & 33 \\
\hline
\end{tabular}

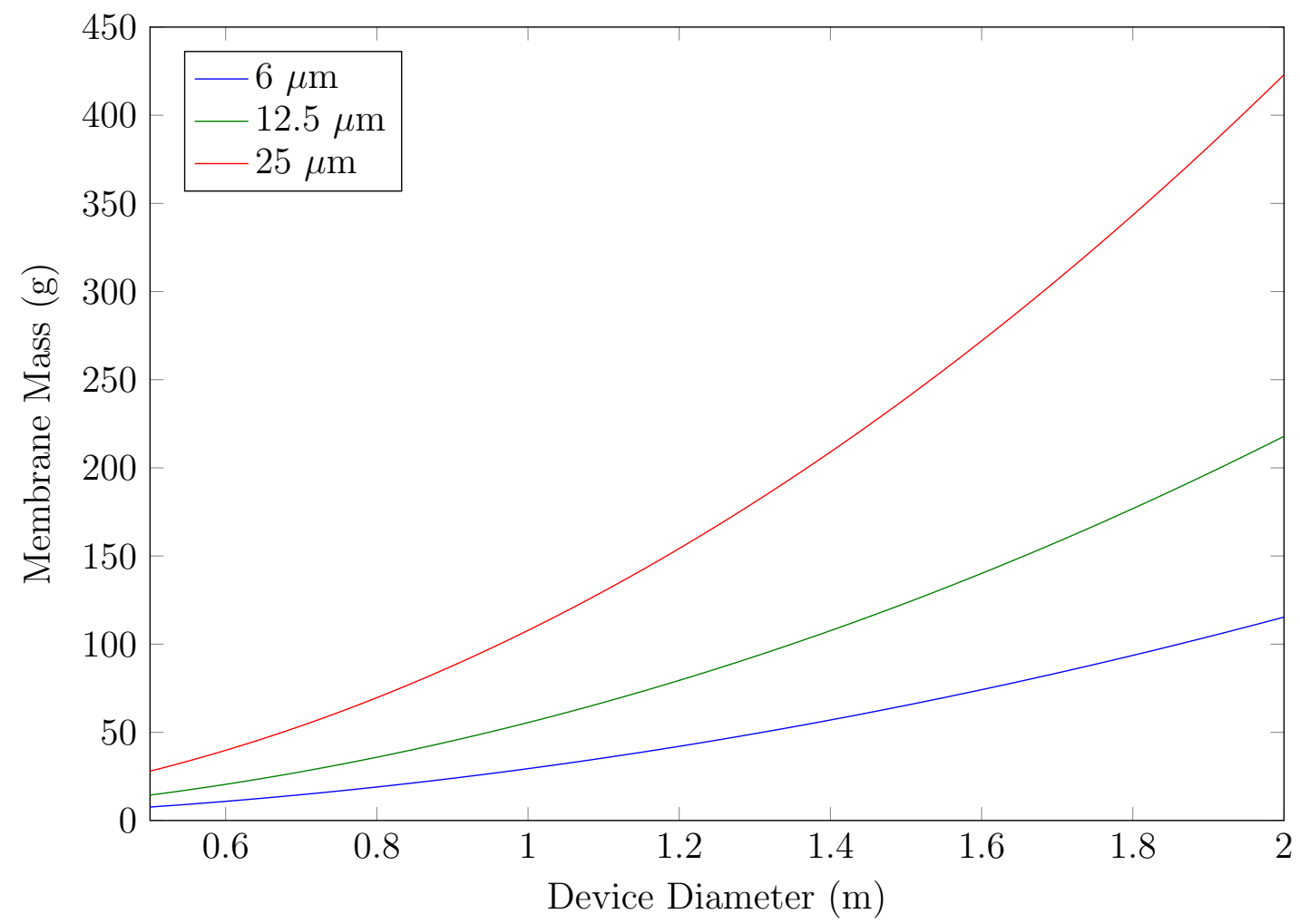

Figure 5.4: Mass of membrane material based in total device diameter.

It is seen that an area to mass ratio of about $1.75 \mathrm{~m}^{2} / \mathrm{kg}$ leads to a diameter, assuming a total spacecraft mass of $1.3 \mathrm{~kg}$, of $1.7 \mathrm{~m}$ device. Seen in Figure 5.4 
and Figure 5.5 that both the $12.5 \mu \mathrm{m}$ and $25 \mu \mathrm{m}$ thick membrane yield very large systems, for this reason the $6 \mu \mathrm{m}$ thick film will be used for analysis. Films which are thinner than $6 \mu \mathrm{m}$, although lighter and utilize less stored volume, will be assumed to be to difficult to work with and will not be considered farther. This results in a film mass of $83.6 \mathrm{~g}$, and a stored volume of $223 \mathrm{~cm}^{3}$.

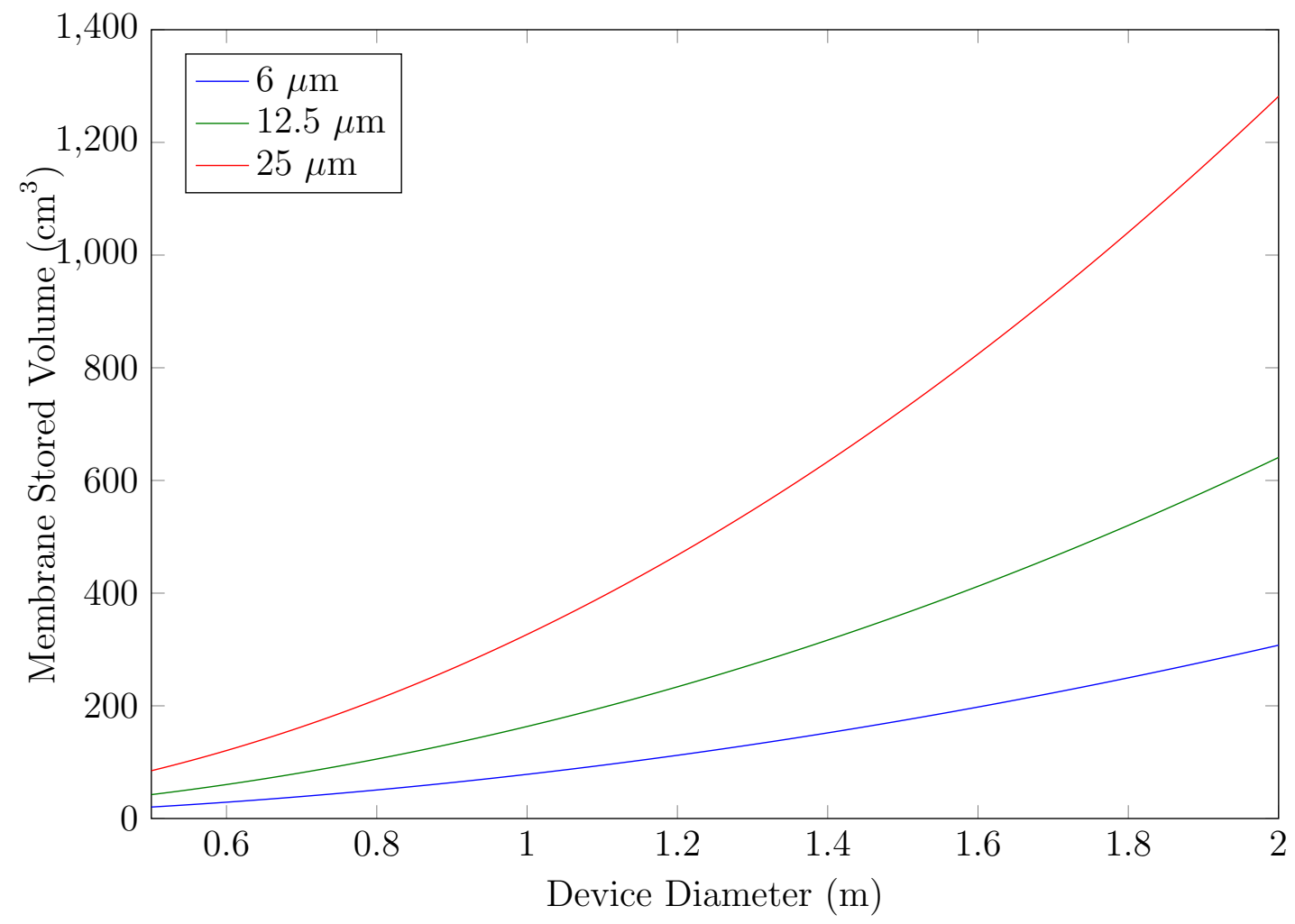

\section{Figure 5.5: Stored volume of membrane material based in total device diameter.}

For a spacecraft with a known launch date a time-dependent atmospheric model is much more accurate option when predicting the orbital lifetime. Using the same atmospheric assumptions in Section 2.2.1, including the assumption that a solar cycle is exactly 11 years, the results of an analysis using the timedependent NRLMSISE-00 atmospheric model can be see in Figure 5.6. The deployment versus reentry date can be seen in Figure 5.7. Seen in 5.7 is that 


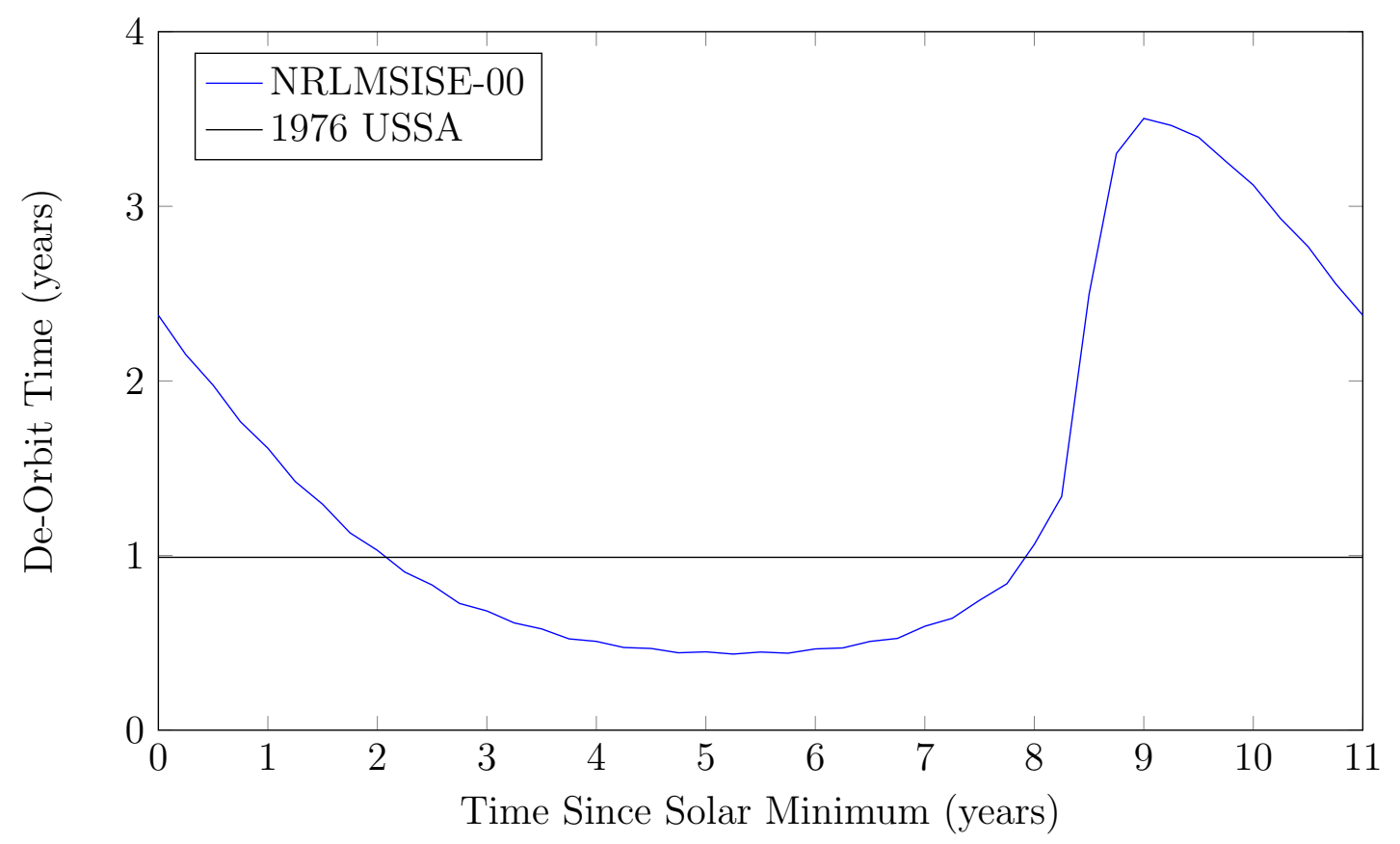

Figure 5.6: De-orbit time based on time since the last solar minimum using the NRLMSISE-00 atmospheric model.

from about 2018 to 2020 there is quite little difference in de-orbit date. This would have major implications for the amount of gas needed by the system to maintain inflation throughout the system's lifetime.

Since this system is designed for a nominal one year de-orbit at an $800 \mathrm{~km}$ orbit, analysis was also performed at a range of attitudes using three atmospheric scenarios: deployment at solar minimum, deployment at solar maximum, and using the nominal USSA 1976 model. These results can be seen in Figure 5.8.

Presented thus far in this chapter are results for a spacecraft beginning at an $800 \mathrm{~km}$ orbit. To state these results can be interpolated or extrapolated to fit any orbit, launch date, or mission requirements is unreasonable. Selected parameters were changed to show general de-orbit time trends, since in reality orbital lifetime has a complex relationship with many different factors. 


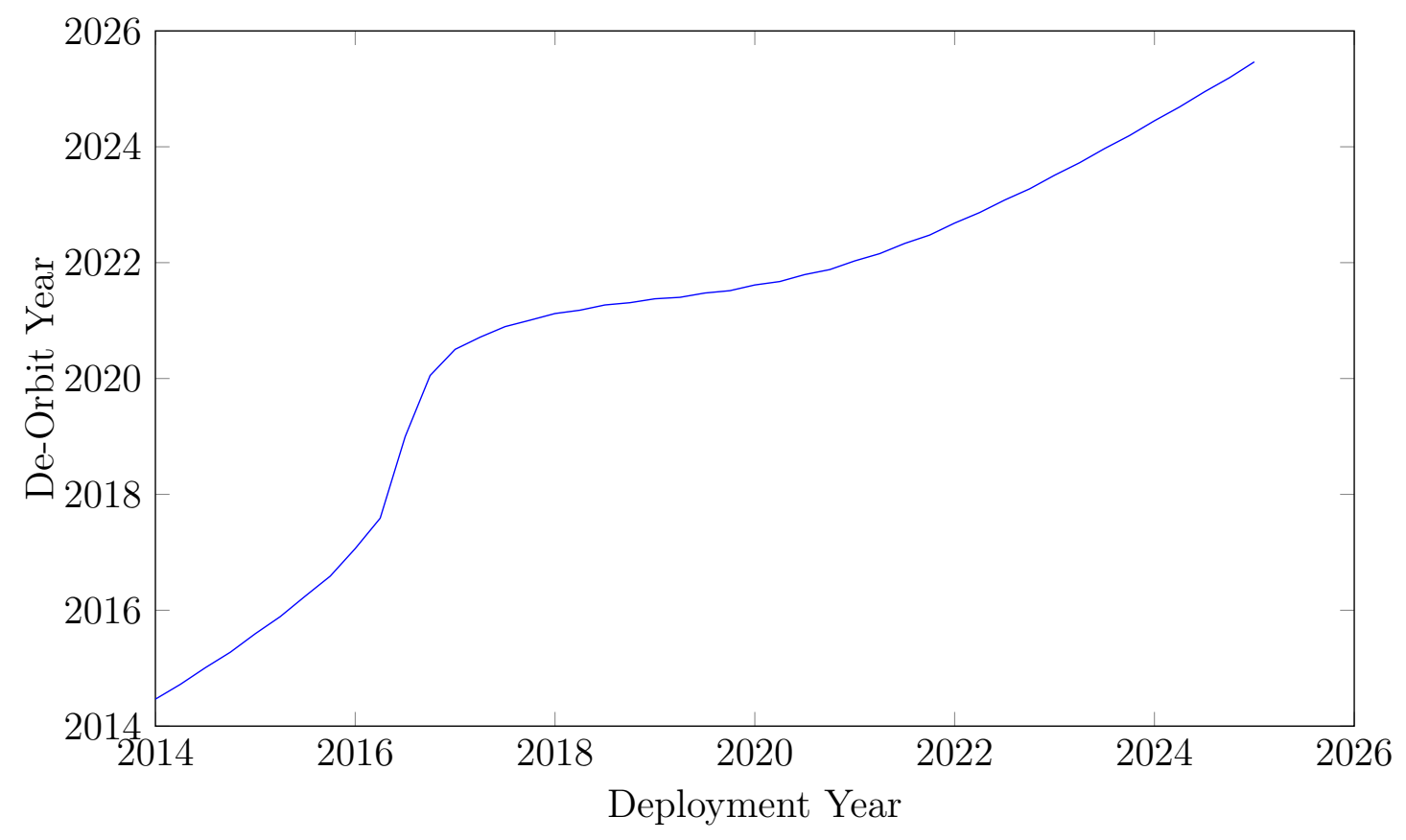

Figure 5.7: Reentry date based on deployment date using the NRLMSISE-00 atmospheric model.

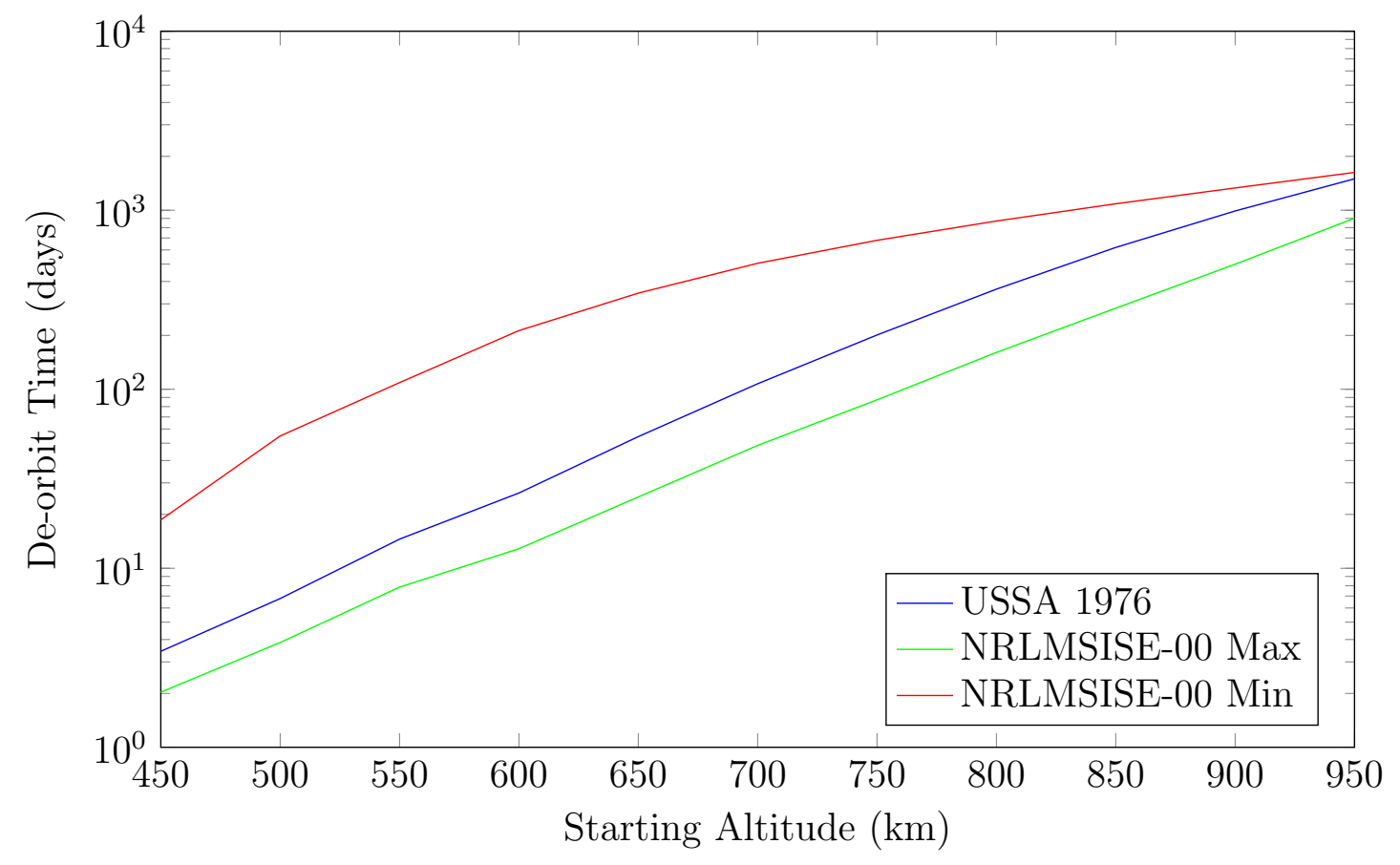

Figure 5.8: Orbital lifetime over a range of altitudes, with three different atmospheric scenarios. 


\subsection{Leak Analysis Results}

Early analysis concluded that the rate of gas leak is exponentially proportional to the spacecraft's altitude. This is because the maximum pressure seen by the inflatable is related to the local atmospheric density. Due to this relationship a "failure altitude" would be used, at which point the system would no longer attempt to maintain proper internal pressure because of the high rate of leakage. At the prescribed point of failure the ballistic coefficient of the spacecraft would be set to a nominal $59 \mathrm{~kg} / \mathrm{m}^{2}$, this massive increase in the ballistic coefficient leads to an increased orbital lifetime. A sweep of different failure altitudes was performed, seen in Figure 5.9 is the resulting orbital lifetime and amount of gas needed to maintain proper inflation until the failure altitude.

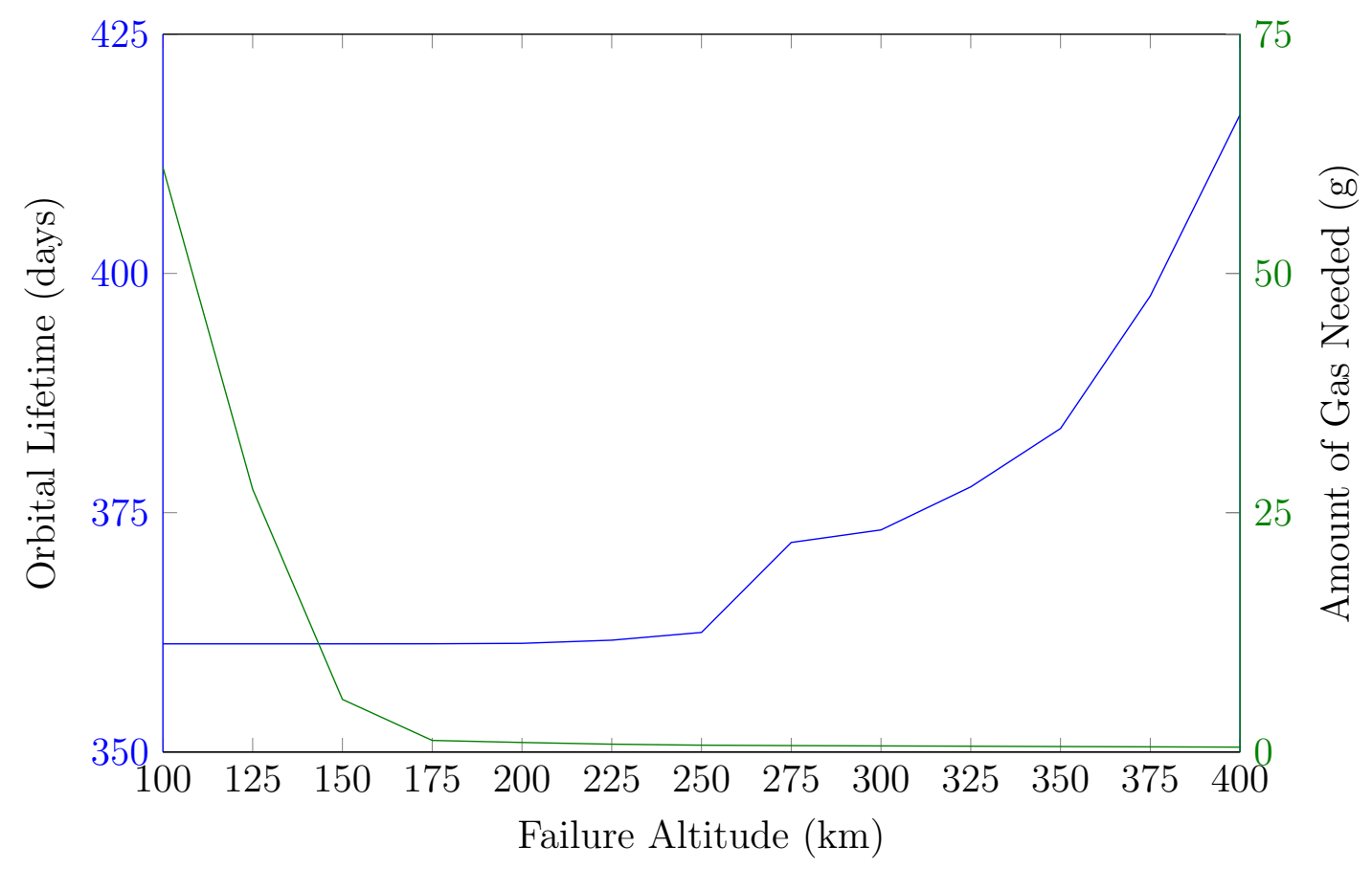

Figure 5.9: System failure altitude and its the resulting orbital lifetime and cumulative inflation gas used. 
From the results displayed in Figure 5.9 that a predetermined system failure altitude of $250 \mathrm{~km}$ is reasonable. This results in an increase orbital lifetime of 1.19 days, but a large decrease in needed inflation gas from $61 \mathrm{~g}$ to $0.7 \mathrm{~g}$. From here on all results will be presented have a set failure altitude of $250 \mathrm{~km}$. An unplanned failure at higher altitudes does greatly affect the orbital lifetime of the CubeSat. Data from Figure 3.2 shows that if failure happens below $700 \mathrm{~km}$, the body will de-orbit within 25 years of that date.

To first calculate the amount of holing accumulated throughout the life of the device, the range of orbital debris and micrometeorites which penetrate one and two sides of the device must be known. From there the possible orbital debris and micrometeorites are broken down into four more regimes which logarithmically increases to $0.1 \mathrm{~mm}$, a mostly arbitrary size but for both orbital debris and micrometeorites that size has a flux of about 1-10 at $800 \mathrm{~km}$. For the resulting hole size the maximum hole size for that particle range was chosen, except for the final particle range which has a flux extending from $0.1 \mathrm{~mm}$ to infinitely large pieces, for this regime the hole size will be the hole for $0.1 \mathrm{~mm}$ particle. A summary of the above information is in Table 5.4 for orbital debris and Table 5.5 for micrometeorites.

Table 5.4: Hole size and particle flux parameters for orbital debris particles.

\begin{tabular}{l|l|l|l}
\hline Flux Regime & Hole Diameter & Hole Area & Number of Holes \\
\hline $0.18 \mu \mathrm{m}-0.37 \mu \mathrm{m}$ & $0.37 \mu \mathrm{m}$ & $0.1 \mu \mathrm{m}^{2}$ & 1 \\
$0.37 \mu \mathrm{m}-1.5 \mu \mathrm{m}$ & $1.5 \mu \mathrm{m}$ & $1.1 \mu \mathrm{m}^{2}$ & 2 \\
$1.5 \mu \mathrm{m}-6.1 \mu \mathrm{m}$ & $6.1 \mu \mathrm{m}$ & $29 \mu \mathrm{m}^{2}$ & 2 \\
$6.1 \mu \mathrm{m}-24 \mu \mathrm{m}$ & $24 \mu \mathrm{m}$ & $425 \mu \mathrm{m}^{2}$ & 2 \\
$24 \mu \mathrm{m}-100 \mu \mathrm{m}$ & $100 \mu \mathrm{m}$ & $7853 \mu \mathrm{m}^{2}$ & 2 \\
$100 \mu \mathrm{m}+$ & $100 \mu \mathrm{m}$ & $7853 \mu \mathrm{m}^{2}$ & 2 \\
\hline
\end{tabular}


Table 5.5: Hole size and particle flux parameters for micrometeorites particles.

\begin{tabular}{l|l|l|l}
\hline Flux Regime & Hole Diameter & Hole Area & Number of Holes \\
\hline $0.12 \mu \mathrm{m}-0.24 \mu \mathrm{m}$ & $0.24 \mu \mathrm{m}$ & $0.05 \mu \mathrm{m}^{2}$ & 1 \\
$0.24 \mu \mathrm{m}-1 \mu \mathrm{m}$ & $1 \mu \mathrm{m}$ & $0.8 \mu \mathrm{m}^{2}$ & 2 \\
$1 \mu \mathrm{m}-4.9 \mu \mathrm{m}$ & $4.9 \mu \mathrm{m}$ & $18 \mu \mathrm{m}^{2}$ & 2 \\
$4.9 \mu \mathrm{m}-22 \mu \mathrm{m}$ & $22 \mu \mathrm{m}$ & $380 \mu \mathrm{m}^{2}$ & 2 \\
$22 \mu \mathrm{m}-100 \mu \mathrm{m}$ & $100 \mu \mathrm{m}$ & $7853 \mu \mathrm{m}^{2}$ & 2 \\
$100 \mu \mathrm{m}+$ & $100 \mu \mathrm{m}$ & $7853 \mu \mathrm{m}^{2}$ & 2 \\
\hline
\end{tabular}

Cumulative hole area for the nominal $800 \mathrm{~km}$ scenario can be seen in Figure 5.10. Although no formal validation of this algorithm was conducted results here scale reasonably well to similar analysis from Global Aerospace Corporation for their analysis of $37 \mathrm{~m}$ diameter device [41].

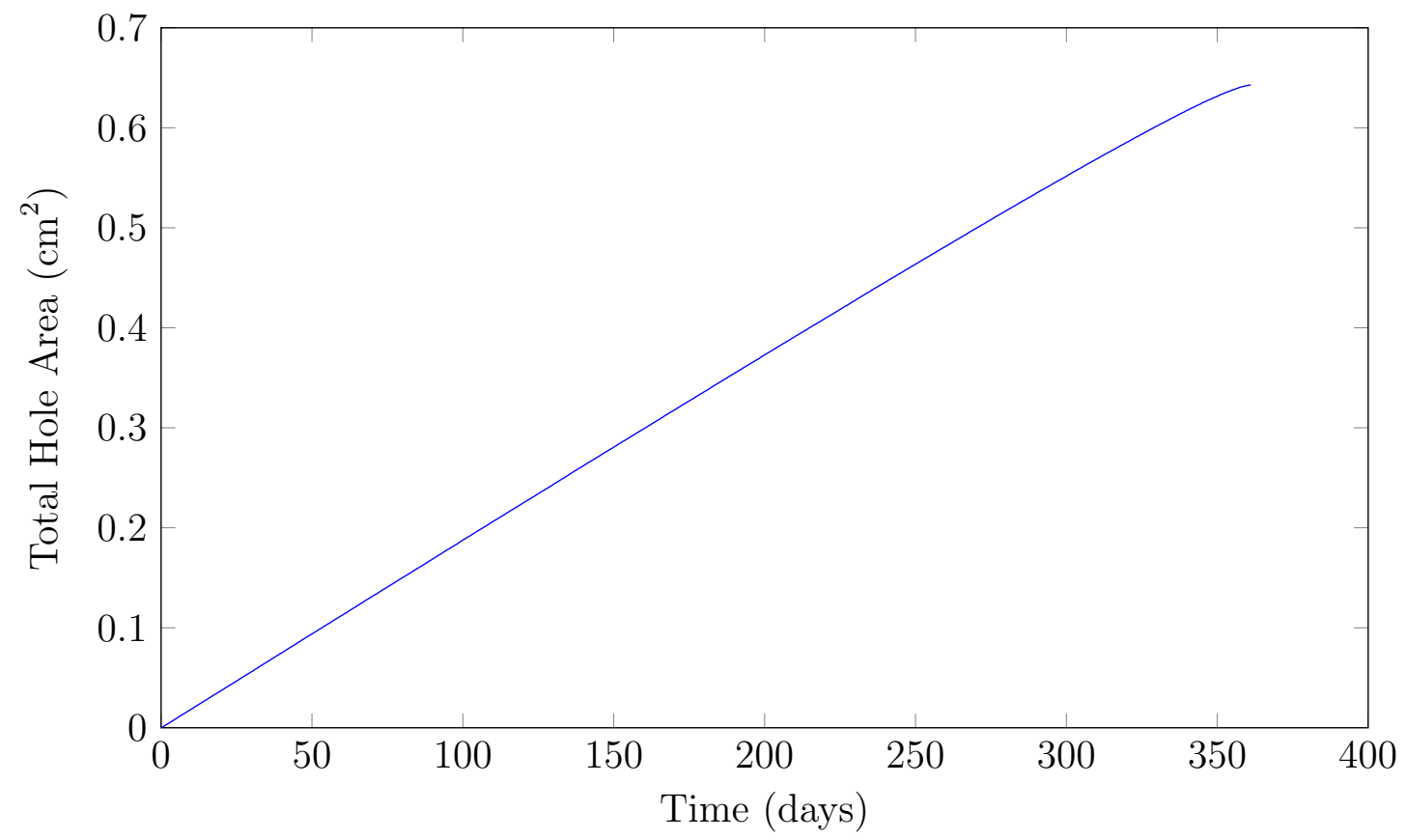

Figure 5.10: Hole area over time for a $1.7 \mathrm{~m}$ diameter device starting at an $800 \mathrm{~km}$ sun-synchronous orbit.

In order to keep the system realistic, a non-perfect inflation system will be 
used to keep pressure in the device. A perfect inflation system is one that can release any amount gas, and since the amount of gas flow rate is sometimes on the order on $\mathrm{ng} / \mathrm{s}$ this is an unrealistic exception. Here it will be assumed that the inflation system can dispense $6 \mu \mathrm{g}$ of gas and above. This number has been determined from a survey of commercial off-the-shelf miniature gas valves. To accomplish this requirement the valve would most likely have a response time on the order of microseconds and may have a screened orifice attached to achieve the desired flow rate. The amount of gas to be released into the device by the inflation system is done by releasing the amount of from the previous time step and multiplying it by some factor. Varying this factor effects the amount of gas needed as well as the number of cycles of the inflation valve. Since the number of necessary valve cycles doesn't exceed a few ten thousand a factor of 2 will be chosen.

It has been shown that for inflatable reentry vehicles a ratio between the internal pressure and dynamic pressure of 12 results in good reentry performance [48]. In this project a pressure ratio of 5-10 will be considered acceptable. Due to the fact that during the majority of the spacecraft's life it is operating in a fully rarefied environment the total pressure, not just dynamic pressure will be used when calculating the pressure ratio. At very high altitudes, rather than the pressure from the atmosphere being of concern, the solar radiation pressure is the dominate pressure. Since in this analysis the sampling of internal pressure is discrete a minimum pressure ratio of 20 will be attempted to be maintained, this is because although at the beginning of a time step the pressure ratio may be above 20 at the end of the time step the pressure ratio may have fallen well below 20 .

The minimum pressure ratio results can be seen in Figure 5.11. For this 


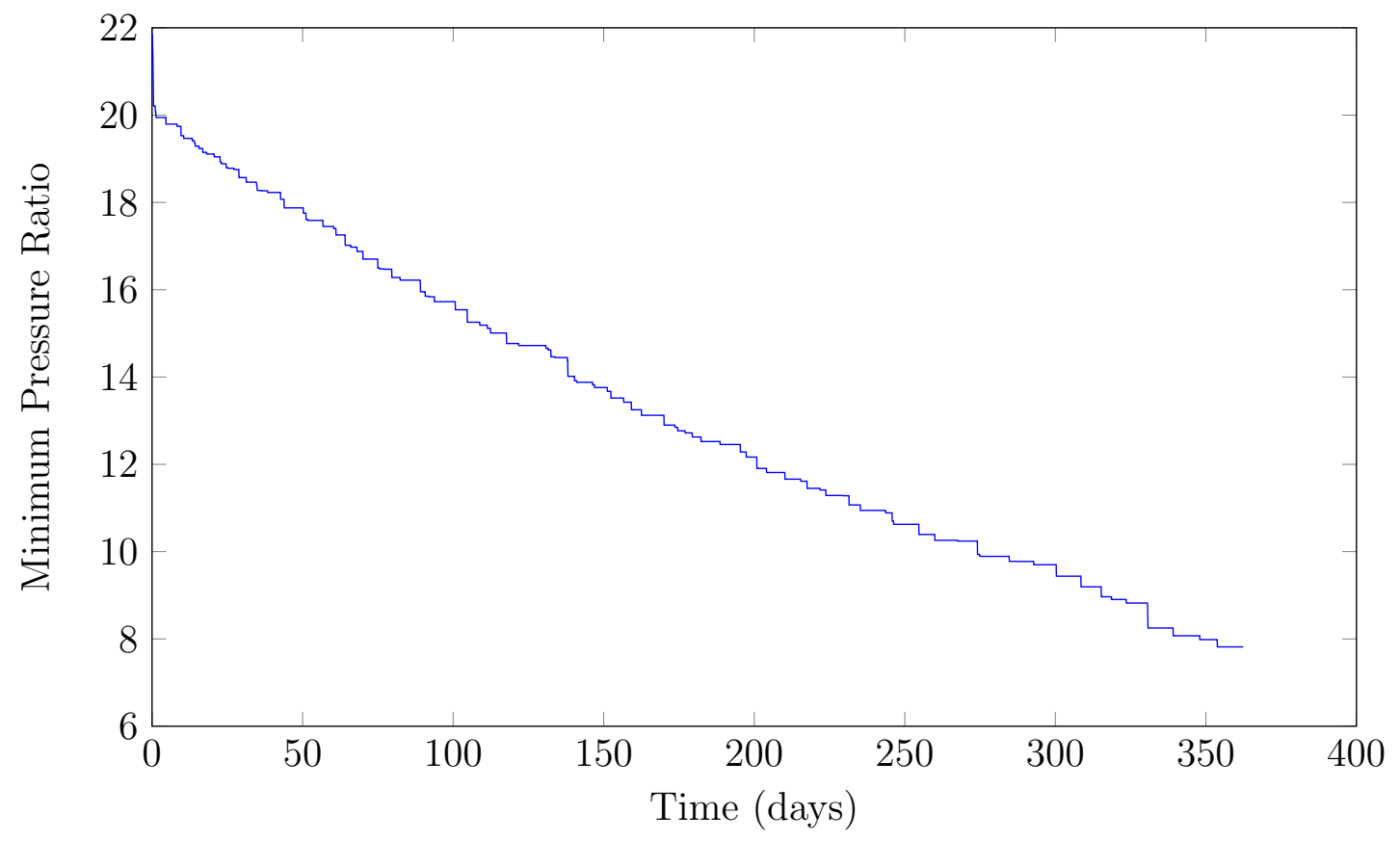

Figure 5.11: Minimum internal to external pressure ratio over time.

analysis the inflating gas will be nitrogen and the temperature of the gas inside the device will be $300 \mathrm{~K}$. Results for cumulative gas used over time in Figure 5.12. It should also be mentioned that since the leak rate is proportional to the internal pressure each time step is broken down into many smaller time steps. This lowers the error caused by the default time step size by between $2 \%$ and $50 \%$.

It can be seen in Figure 5.12 that towards the end of the spacecraft's life that the amount of gas necessary to maintain inflation is rapidly increasing. As mentioned earlier this is the reason that a failure altitude is needed. As shown in 5.9 , as long as the failure altitude is below $250 \mathrm{~km}$, little performance is lost while the mass savings is great.

From the time-based atmospheric model results seen in Figure 5.6 the resulting necessary gas for the mission can be seen in Figure 5.13. Also from the 


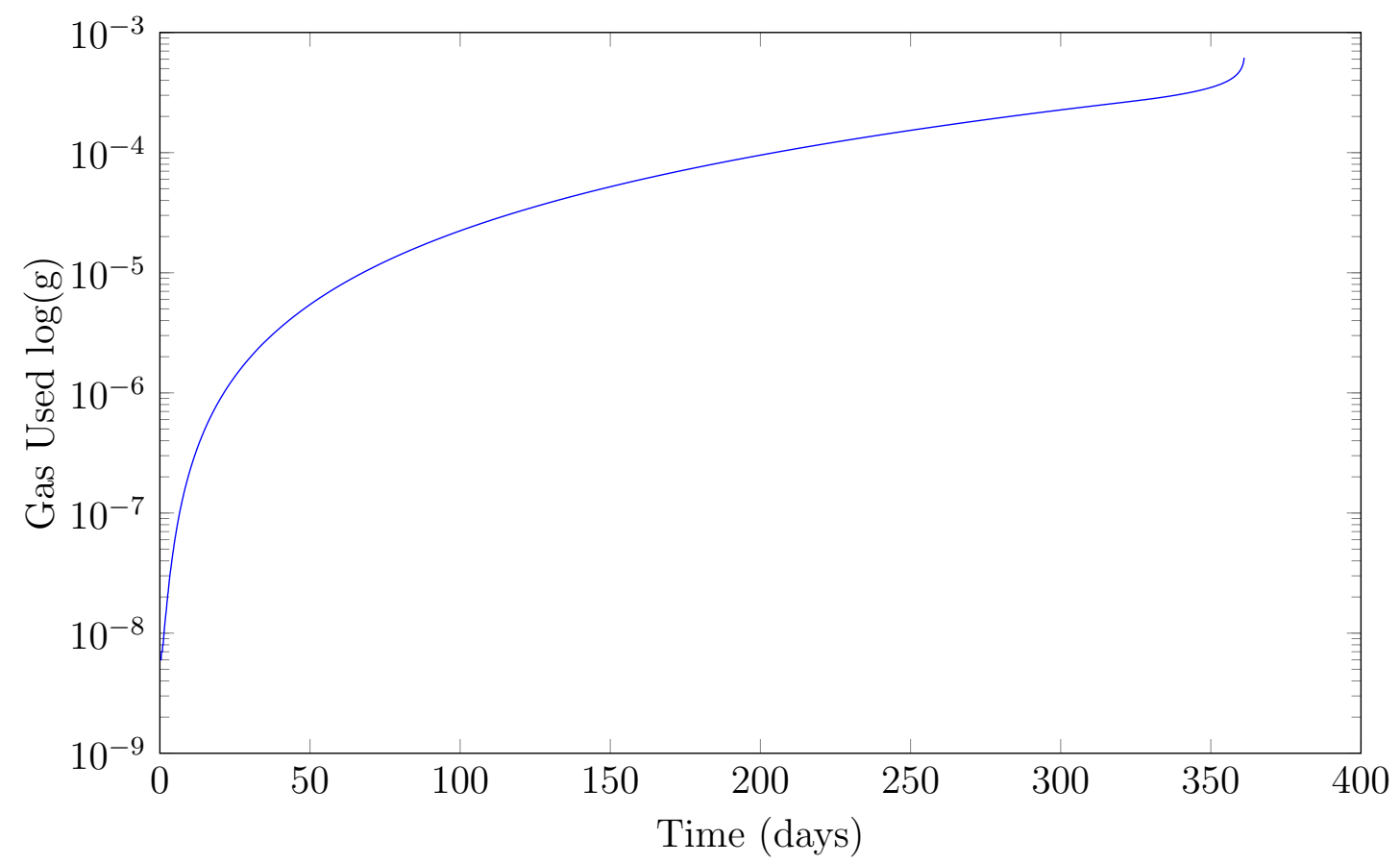

Figure 5.12: Cumulative gas used over time for a $1.7 \mathrm{~m}$ diameter device starting at an $800 \mathrm{~km}$ sun-synchronous orbit in 2013.

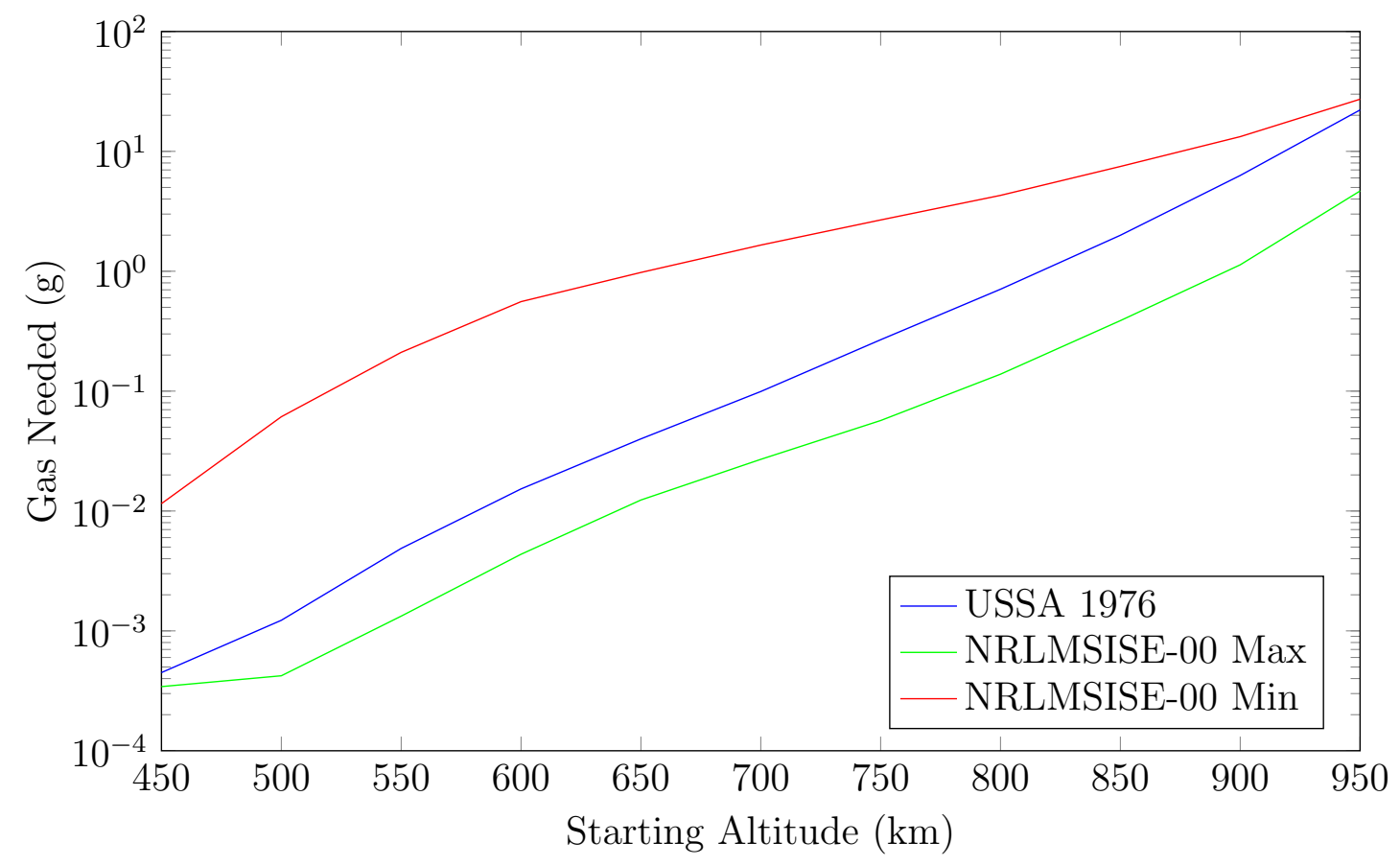

Figure 5.13: Gas requirements over a range of altitudes, with three different atmospheric scenario. 
altitude sweep seen in Figure 5.6 the accompanying inflating gas required can be viewed in Figure 5.14. It can be seen in Figure 5.14 that much less could be required if the deployment date is more towards solar maximum.

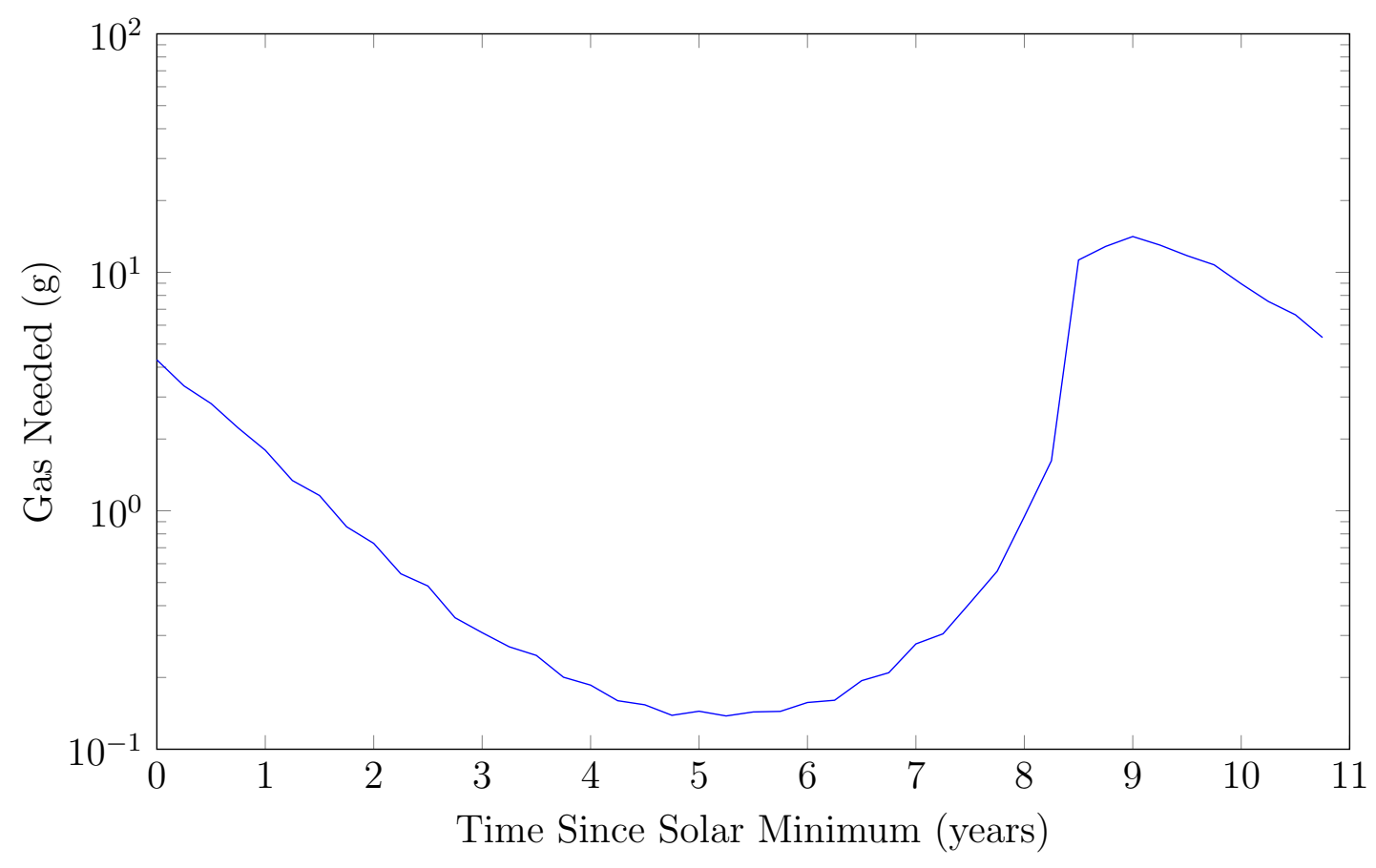

Figure 5.14: Gas requirements based on deployment date.

\subsection{System Design}

The goal of this design is to fit the complete device inside of a $1 \mathrm{U}$, taking up minimal volume and mass. In an attempt to comply with CubeSat specification 2.1.4 which states "no pressure vessels over 1.2 standard atmosphere shall be permitted" [8] gas will be stored as an inert solid in the form of a cool gas generator. A micro cold gas generator has be developed by CCG Technologies [49] carries an amount of stored inert nitrogen gas in a small cylinder until released, its parameters can be found in Table 5.6, and is seen in Figure 5.15. 
Table 5.6: Miniaturized cool gas generator parameters [49].

\begin{tabular}{l|l}
\hline Parameter & Value \\
\hline System Mass & $2 \mathrm{~g}$ \\
Diameter & $12 \mathrm{~mm}$ \\
Length & $30 \mathrm{~mm}$ \\
Stored Gas & $120 \mathrm{mg}$ \\
Temperature Rage & $-10 \mathrm{C}$ to $40 \mathrm{C}$ \\
Maximum Pressure & $10 \mathrm{bar}$ \\
\hline
\end{tabular}

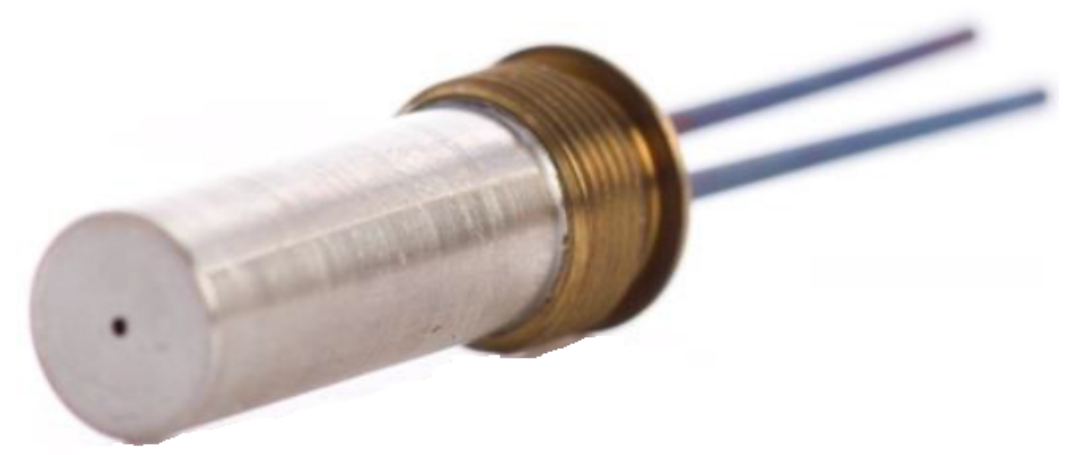

Figure 5.15: Miniaturized cool gas generator by CCG Technologies [49].

Given the gas requirement of $0.7 \mathrm{~g}$, and that a single ccg can provide $0.12 \mathrm{~g}$ of nitrogen gas, seven ccgs are required for the duration of the mission. It will be assumed that a suitable valve will weigh no more than $40 \mathrm{~g}$ and have dimensions of $30 \mathrm{~mm}$ long with a diameter of $12 \mathrm{~mm}$. One of the sides of the CubeSat will have to be opened to allow the device to deploy. To accomplish this hinge and deployment mechanisms were allowed volume inside of the thin-film storage compartment, both of these volumes are allowed a volume of $5 \times 7.8 \times 1 \mathrm{~cm}$. As a note: any hinge mechanism which protrudes from the surface of the CubeSat must comply with CubeSat standard 2.2.6 which states: "all components shall not exceed $6.5 \mathrm{~mm}$ normal to the surface of the $100.0 \mathrm{~mm}$ cube" [8]. A separate 
gas storage compartment is seated underneath of this area. This volume must be completely sealed and air-tight as it serves as temporary storages for gas released from a CCG and is awaiting entry into the inflatable device. A valve connects the stored gas reservoir to the inflatable via a line which must penetrate the wall of the vessel. A summary of dimensions and total mass can be seen in Table 5.7.

Table 5.7: System design results.

\begin{tabular}{l|l}
\hline Parameter & Value \\
\hline Total Depth & $5 \mathrm{~cm}$ \\
Width & $10 \mathrm{~cm}$ \\
Height & $10 \mathrm{~cm}$ \\
Membrane Mass & $84 \mathrm{~g}$ \\
Valve Mass Allowance & $40 \mathrm{~g}$ \\
Complete Gas Mass & $14 \mathrm{~g}$ \\
Structure Mass & $190 \mathrm{~g}$ \\
Total Mass & $328 \mathrm{~g}$ \\
\hline
\end{tabular}

Images of the system can be seen in Figure 5.16, Figure 5.17, and Figure 5.18. Note that the figures show a total of eight ccgs and two valves, this is just to show more components can fit inside the volume and does not match up with the mass values given in Table 5.7.

The mass of the lid (top), hinge, and release mechanisms are not accounted for in Table 5.7. It was also assumed that the density of the structures material is $2.7 \mathrm{~g} / \mathrm{cm}^{3}$. A matter of concern is the amount of pressure inside of the gas containment volume. With seven ccgs and one valve the free volume inside of the gas container is about $27.1 \mathrm{~cm}^{3}$ leading to an pressure of about $0.92 \mathrm{~atm}$, assuming a temperature of 20 degrees Celsius. If all 10 available slots are filled, as shown in Figure 5.17, the pressure would be as high as $0.98 \mathrm{~atm}$.

It should be stated here that the design is nothing more than a proof of concept design. It is meant only to give the scientific and engineering community 


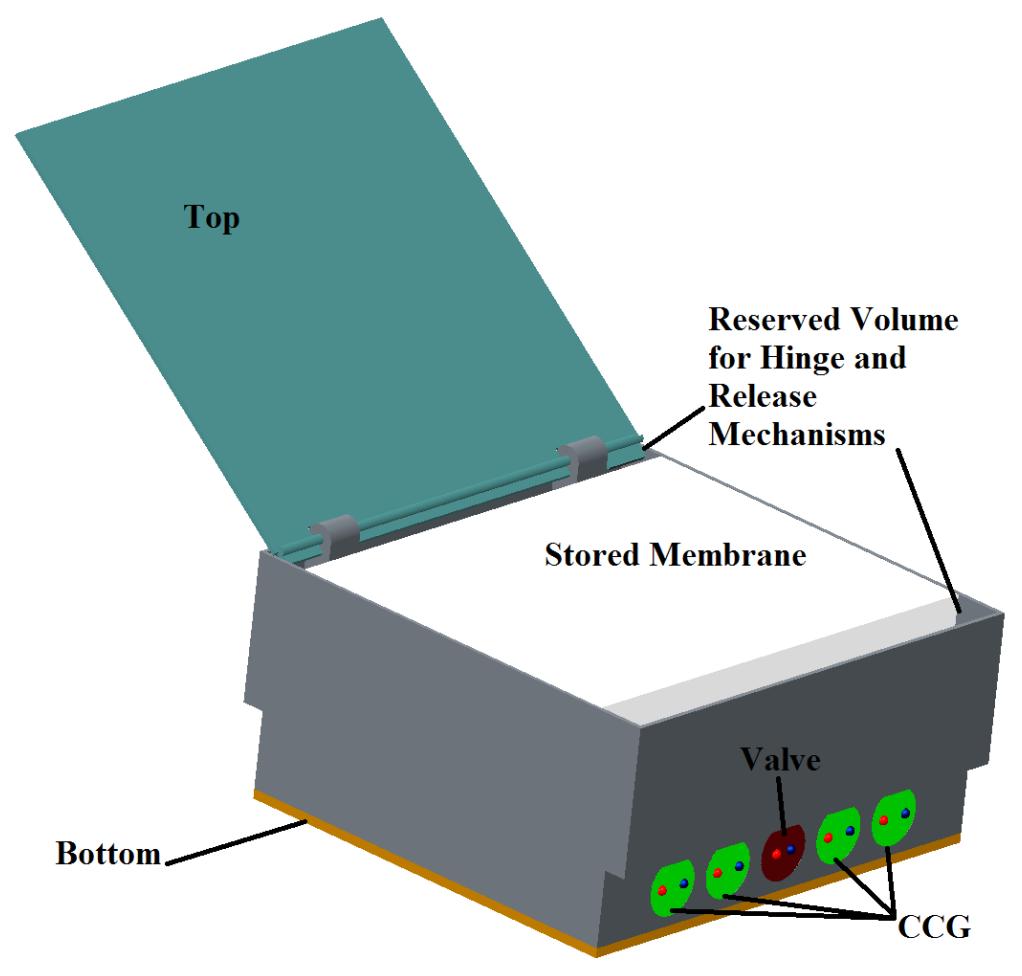

Figure 5.16: System with top released.

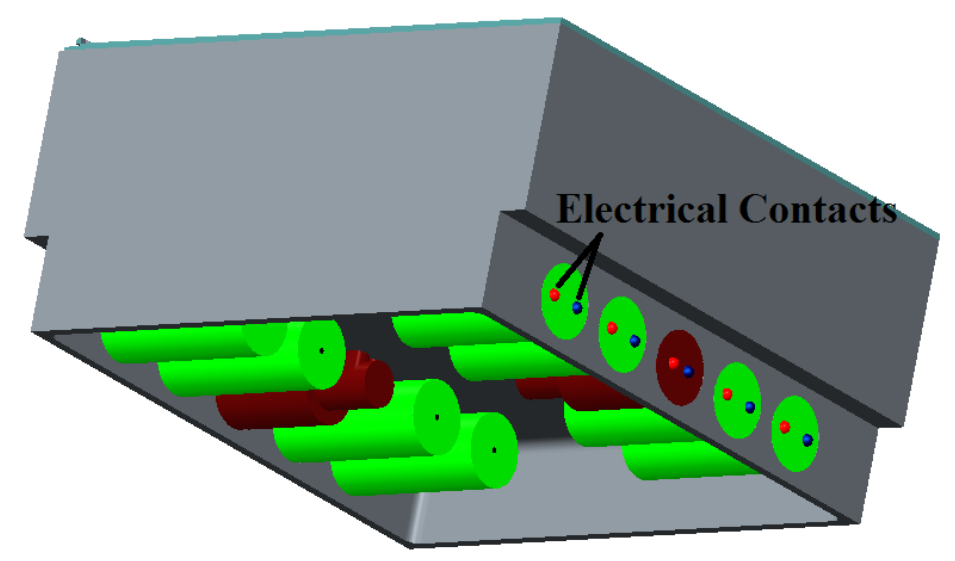

Figure 5.17: System seen from the bottom. 


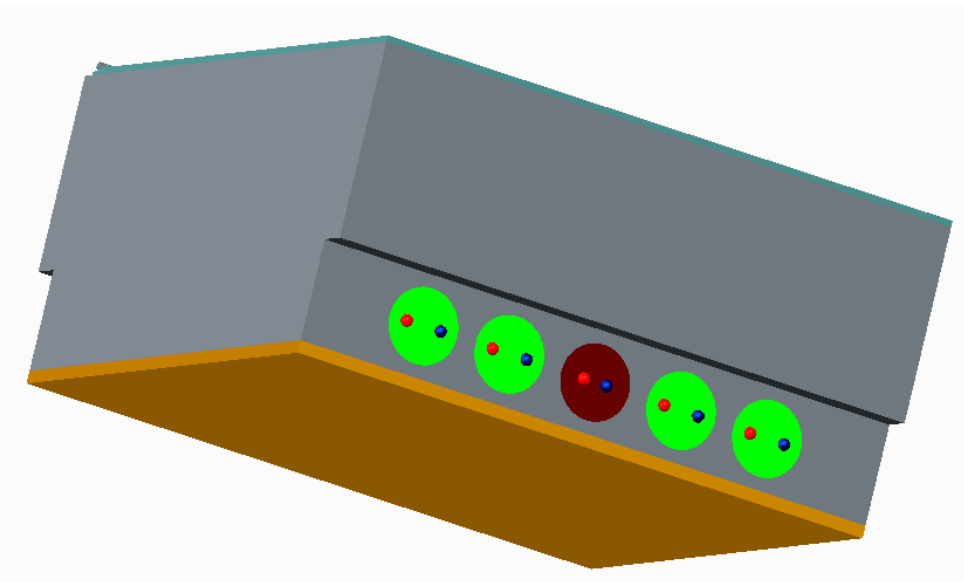

Figure 5.18: System in stored configuration.

an idea of what such a system of this size might look like.

\subsection{Comparison with a 2-D Sail}

Nanosail D-2 deployed its $10 \mathrm{~m}^{2}$ sail January $19^{\text {th }}$, 2010. The $4 \mathrm{~kg}$ started in a $641 \times 652 \mathrm{~km}$ orbit at an inclination of 72 degrees [35]. The spacecraft was expected to de-orbit in 70-120 days and actually reentered after 240 days [36]. It can be safe to assume the spacecraft was not pointing in the nominal velocityfacing direction for the entirety of its life. For a tumbling body attached to a flat plate, which is sufficiently larger than the body, the average cross sectional area approaches about $40 \%$ of the area of the plate. From this additional calculations were performed to estimate the orbital lifetime of a tumbling Nanosail D-2. From these calculations the discrepancy between estimated and actual de-orbit times can be accounted for, these values can be seen in Table 5.8. 
Table 5.8: Nanosail D-2 de-orbit values [36].

\begin{tabular}{l|l}
\hline Scenario & De-Orbit Time (days) \\
\hline 2-D Optimal Pointing & $70-120$ \\
2-D Tumbling & $170-293$ \\
Actual & 240 \\
\hline
\end{tabular}

\subsubsection{Effectiveness}

To compare the effectiveness a brief discussion on area-time-product (ATP) is in order. ATP is simply the cross sectional area times the orbital lifetime, this is important because it can be directly related the probably impact with orbital debris and creating more debris. Since mass is important to spacecraft engineers the term which will determine the effectiveness of a device is the area-time-massproduct (ATMP), simply given by:

$$
\text { ATMP }=\text { Area } \times \text { OrbialLifetime } \times \text { Mass }
$$

Since CP-5 contained a 2-D drag sail device sized to be used in a $1 \mathrm{U}$ CubeSat its estimated effectiveness at an $800 \mathrm{~km}$ orbit will be compared to the effectiveness of the system presented here. Although the mass of the sail subsystem was not found in publications, the author was able to measure and weigh the CP5 engineering model here at Cal Poly. Unfortunately, at the time of measurement the engineering model was not accompanied by the sail or its booms. The measurement values can be seen in Table 5.9.

As discussed in Section 3.4 magnetic tethers can be a very effective de-orbit technique. A CubeSat sized system has been proposed by Tethers Unlimited. At an initial altitude of $800 \mathrm{~km}$ their data shows that a $1 \mathrm{U}$ CubeSat can be de-orbited in a year and a half. Unfortunately, this data does not state at which 
Table 5.9: CP5 Measurements.

\begin{tabular}{l|l}
\hline Measurement & Value \\
\hline Wall Thickness & $2 \mathrm{~mm}$ \\
Exterior Width & $8.2 \mathrm{~cm}$ \\
Exterior Depth & 8.2 \\
Total Height & $6.2 \mathrm{~cm}$ \\
Total Volume & $416 \mathrm{~cm}^{2}$ \\
Mass & $216 \mathrm{~g}$ \\
\hline
\end{tabular}

inclination this analysis assumed. As shown in Figure 3.6 the performance of an electromagnetic tether is dependent on inclination. At high inclinations, such as at a Sun-synchronous orbit, the effectiveness is highly degraded. Additionally, it is assumed that the tether is not severed by micrometeorite or orbital debris strike. Shown in Table 5.10 are the comparisons of several PMD methods. The Terminator Tape row in this table represents a best-case scenario.

Table 5.10: Effectiveness of an inflatable device versus other PMD systems [50].

\begin{tabular}{l|l|l|l|l}
\hline System & Orbital Lifetime & Mass & ATP & ATMP \\
\hline Inflatable & $0.993 \mathrm{yr}$ & $328 \mathrm{~g}$ & $2.254 \mathrm{~m}^{2} \mathrm{yr}$ & $739.3 \mathrm{~m}^{2} \mathrm{yr} \mathrm{g}$ \\
\hline Sail (Tumbling) & $5.268 \mathrm{yr}$ & $216 \mathrm{~g}$ & $5.268 \mathrm{~m}^{2} \mathrm{yr}$ & $1138 \mathrm{~m}^{2} \mathrm{yr} \mathrm{g}$ \\
\hline Sail (Controlled) & $2.211 \mathrm{yr}$ & $216 \mathrm{~g}$ & $2.211 \mathrm{~m}^{2} \mathrm{yr}$ & $477.6 \mathrm{~m}^{2} \mathrm{yr} \mathrm{g}$ \\
\hline Terminator Tape & $1.5 \mathrm{yr}$ & $83 \mathrm{~g}$ & $0.023 \mathrm{~m}^{2} \mathrm{yr}$ & $1.91 \mathrm{~m}^{2} \mathrm{yr} \mathrm{g}$ \\
\hline No PMD & $150-1500 \mathrm{yr}$ & $\mathrm{N} / \mathrm{A}$ & $2.3-23 \mathrm{~m}^{2} \mathrm{yr}$ & $\mathrm{N} / \mathrm{A}$ \\
\hline
\end{tabular}

A more in-depth study of different de-orbit techniques has been conducted by the Global Aerospace Corporation and can be viewed in their Journal of Spacecraft and Rockets paper Removing Orbital Debris with Less Risk [41]. One important distinction made in the paper is the difference between high-energy collisions and low-energy collisions. Simply, a collision is said to be high-energy if the collision occurs between two rigid structures and low-energy if at least 
one of the incident structures are relatively non-rigid. High-energy collisions are much more dangerous to the orbital debris environment because the result of these collisions is the fragmentation of the two incident bodies. This distinction is important on large systems designed for full-sized spacecraft, however it was not felt to be of much importance for spacecraft of this size since any spacecraft that would collide with a CubeSat's inflatable device is quite likely to strike the CubeSat itself. 


\section{Conclusion}

It is work mentioning again that this project is not only about efficiently deorbiting CubeSats, but to show that this technology can be used to de-orbit larger spacecraft and rocket bodies. As seen in Figure 1.1 the majority of objects in Earth orbit are fragmentation debris, fragmentation from spacecraft and rocket bodies. Removing these hazardous objects from space as soon after their useful lifetime is over is best thing that can be done for the orbital debris environment short of ADR measures.

\subsection{Future Work}

The author believes that this work is a great start towards the possible use of inflation-based methods of PMD. More work is required before inflatable devices may be considered a genuine method of PMD.

With much work conducted in the areas of orbits and MM/OD impact analysis the it is believed that further leak analysis should be conducted. The leak from MM/OD impacts was a great start but the author is concerned about gas leakage from the gas storage container. Also the author believes that the assumption that the impact of very small MM/OD particles do not decompose and does not positivity effect the internal pressure of the envelope may be too conservative of an assumption. 
The system design presented here is useful to gage the size a device such as this may be. Further detail design is required before such a system is to be taken as a genuine method of post-mission disposal. The reliability of the system here has been called into question. Further work on the reliability of the system is required, in particular the lifetime of very small valves.

A "less active" method of inflation would most likely be required of such a system, especially on a CubeSat-sized device. One possible solution is the use of a variable flow rate valve instead of a two-way valve which must be commanded to open and close many thousands of times.

It is worth mentioning again that the analysis presented in the previous chapter is for one particular orbit. If the reader wishes, a copy of the MATLAB code used to perform the analysis presented in this project may be obtained by contacting the Cal Poly Aerospace Engineering Department at aero@calpoly.edu. 


\section{BIBLIOGRAPHY}

[1] Kessler, D. J. and Cour-Palais, B. G., "Collision Frequency of Artificial Satellites: The Creation of a Debris Belt," Journal Of Geophysical Research, Vol. 89, No. A6, June 1979.

[2] Liou, J., "An active debris removal parametric study for LEO environmnet remediation," Advances in Space Research, March 2011.

[3] "Orbital Debris Quarterly News," January 2013.

[4] "Handbook for Limiting Orbital Debris," July 2008.

[5] "NASA Procedural Requirements for Limiting Orbital Debris," May 2009.

[6] "Process for Limiting Orbital Debris," December 2011.

[7] Vallado, D. A., Fundamentals of Astrodynamics and Applications, Microcosm Press, thrid ed., 2007.

[8] "CubeSat Design Specification," August 2009.

[9] Woellert, K., Ehrenfreund, P., Ricco, A. J., and Hertzfeld, H., "Cubesats: Cost-effective science and technology platforms for emerging and developing nations," Advances in Space Research, October 2010.

[10] Curtis, H. D., Orbital Mechanics for Engineering Students, Elsevier, 2nd ed., 2009. 
[11] Fortescue, P., Stark, J., and Swinerd, G., editors, Spacecraft Systems Engineering, Wiley, 2003.

[12] Hawkins, R. and Palomares, J., De-Orbiting Upper Stage Rocket Bodies Using a Deployable High Altitude Drag Sail, Senior project, California Polytechnic State University, 2012.

[13] Tribble, A. C., The Space Environment: Implication for Sacecraft Design, Princeton University Press, 1995.

[14] Mehta, P. M., McLaughlin, C. A., and Sutton, E. K., "Drag coefficient modeling for grace using direct simulation monte carlo," Advances in Space Research, 2013.

[15] Kenneth Moe, M. M. M., "Gassurface interactions and satellite drag coefficients," Planetary and Space Science, 2005.

[16] Walker, A. C., Mehta, P. M., and Koller, J., "A Quasi-Specular Drag Coefficient Model using the Cercignani-Lampis-Lord Gas-Surface Interaction Model," Journal of Spacecraft and Rockets.

[17] Bird, G. A., Visual DSMC Program for Two-Dimensional and Axially Symmetric Flows: The DS2V Program User's Guide, G.A.B Consulting pty Ltd., 144/110 Sussex Street, Sydney NSW 2000, Australla, 3rd ed., October 2006.

[18] Volkov, A., "Direct Simulation Monte Carlo (DSMC) of gas flows," Tech. rep., University of Virginia, Department of Materials Science and Engineering, 2011.

[19] Walker, A. C., Mehta, P. M., and Koller, J., "A Comparison of Different Implementations of Diffuse Reflection with Incomplete Accommodation for Satellite Drag Coefficient Modeling," Planetary and Space Science. 
[20] Pilinski, M., "Satellite Aerodynamic Drag in Earth's Upper Atmosphere," Astrodynamics and Satellite Navigation Seminar, October 2010.

[21] Walker, A., "IMPACT Project: DSMC Modeling of Drag Coefficients," Tech. rep., Los Alamos National Laboratory.

[22] Pilinski, M. D., Dynamic Gas-Surface Interaction Modeling for Satellite Aerodynamic Computations, Ph.D. thesis, University of Colorado, 2011.

[23] Pilinski, M. D., Argrow, B. M., Palo, S. E., and Bowman, B. R., "SemiEmpirical Satellite Accommodation Model for Spherical and Randomly Tumbling Objects," Journal Of Spacecraft And Rockets, Vol. 50, No. 3, May 2013.

[24] "HORIZONS System," 2013.

[25] Streetman, B. and Peck, M. A., "New Synchronous Orbits Using the Geomagnetic Lorentz Force," JOURNAL OF GUIDANCE, CONTROL, AND DYNAMICS, Vol. 30, No. 6, November 2007.

[26] "U.S. Government Orbital Debris Mitigation Standard Practices," Febriary 2001.

[27] Orbital Debris: A Technical Assessment, National Academy Press, 1995.

[28] "Orbital Debris Quarterly News," October 2013.

[29] Forward, R. L., Hoyt, R. P., and Uphoff, C. W., "Terminator Tether: A Spacecraft Deorbit Device," Journal of Spacecraft and Rockets, Vol. 37, No. 2, March 2000.

[30] Hoyt, R., Slostad, J., and Twiggs, R., "The Multi-Application Survivable Tether (MAST) Experiment," Tech. rep., Tethers Unlimited, Inc., 2003. 
[31] Hoyt, R. and Forward, R., "The Terminator Tether: Autonomous Deorbit of LEO Spacecraft for Space Debris Mitigation," American Institute of Aeronautics and Astronautics, 2000.

[32] Bilimoria, K. D. and Krieger, R. A., "Slot architecture for separating satellites in sun-synchronous orbits," Proceedings of the AIAA SPACE Conference and Exposition, 2011.

[33] "PolySat CP5," Internet.

[34] Alhorn, D. C., Casas, J. P., Agasid, E. F., Adams, C. L., Laue, G., Kitts, C., and OBrien, S., "NanoSail-D: The Small Satellite That Could!" 25th Annual AIAA/USU Conference on Small Satellites.

[35] "NanoSail D," Internet.

[36] "NASA's Nanosail-D 'Sails' Home - Mission Complete," Internet, November 2011.

[37] Romagnoli, D. and Theil, S., "De-orbiting satellites in LEO using solar sails," Journal of Aerospace Engineering, Sciences and Applications, Vol. IV, No. 2, 2012, pp. 49-59.

[38] Montgomery, E. E. and Adams, C. L., "NanoSail-D," 2008 CubeSat Developers Workshop, April 2008.

[39] Maessen, D. C., "iDod: Development of a generic inflatabel de-orbit device for CubeSats," Thesis presentation, Delft University of Technology, May 2007.

[40] Nock, K. T., Gates, K. L., Aaron, K. M., and McRonald, A. D., "Gossamer 
Orbit Lowering Device (GOLD) for Safe and Efficient De-orbit," American Institute of Aeronautics and Astronautics, August 2010.

[41] Nock, K. T., Aaron, K. M., and McKnight, D., "Removing Orbital Debris with Less Risk," Journal of Spacecraft and Rockets, January 2013.

[42] Krisko, P., "NASA's bew irvutal debris engineering model, ORDEM2010," Tech. rep., National Aeronautics and Space Administration.

[43] Kessler, D. J., Reynolds, R. C., and Anz-Meador, P. D., "Orbital Debris Environment for Spacecraft Designed to Operate in Low Earth Orbit," Technical Memorandum 100471, National Aeronautics and Space Administration, April 1989.

[44] Meshishnek, M. J., "Overview of the Space Debris Enviroment," Technical Report TR-95(4131)-3, The Aerospace Corporation, March 1995.

[45] Sawle, D. R., "Hypervelocity Impact in Thin Sheets and Semi-Infinite Targets at $15 \mathrm{~km} / \mathrm{sec}$," AIAA Journal, Vol. 8, No. 7, 1970.

[46] Dunmore Corporation, Engineered Films.

[47] Sharipov, F., "Transient Flow of Rarefiend Gas Through an Orifice," The Journal of Vacuum Science and Technology, 2012.

[48] Dillman, R., "Flight Performance of the Inflatable Reentry Vehicle Experiment II," 7th International Planetary Probe Workshop, NASA Langley Research Center, 2010.

[49] CGG technologies, Micro CGG, 2013.

[50] "CubeSat Terminator Tape: Affordable End-of-Mission Deorbit Modeule for CubeSats," . 


\section{A. Data Tables}

Table A.1: U.S. Standard Atmosphere 1976 [7].

\begin{tabular}{c|lll}
\hline Altitude Regime $(\mathrm{km})$ & Base Altitude $(\mathrm{km})$ & Base Density $\left(\mathrm{kg} / \mathrm{m}^{3}\right)$ & Scale Height $(\mathrm{km})$ \\
\hline $100-110$ & 100 & $5.297 \mathrm{e}-7$ & 5.877 \\
\hline $110-120$ & 110 & $9.661 \mathrm{e}-8$ & 7.263 \\
\hline $120-130$ & 120 & $2.438 \mathrm{e}-8$ & 9.473 \\
\hline $130-140$ & 130 & $8.484 \mathrm{e}-9$ & 12.636 \\
\hline $140-150$ & 140 & $3.845 \mathrm{e}-9$ & 16.149 \\
\hline $150-180$ & 150 & $2.070 \mathrm{e}-9$ & 22.523 \\
\hline $180-200$ & 180 & $5.464 \mathrm{e}-10$ & 29.740 \\
\hline $200-250$ & 200 & $2.789 \mathrm{e}-10$ & 37.105 \\
\hline $250-300$ & 250 & $7.248 \mathrm{e}-11$ & 45.546 \\
\hline $300-350$ & 300 & $2.418 \mathrm{e}-11$ & 53.628 \\
\hline $350-400$ & 350 & $9.518 \mathrm{e}-12$ & 53.298 \\
\hline $400-450$ & 400 & $3.725 \mathrm{e}-12$ & 58.515 \\
\hline $450-500$ & 450 & $1.585 \mathrm{e}-12$ & 60.828 \\
\hline $500-600$ & 500 & $6.967 \mathrm{e}-13$ & 63.822 \\
\hline $600-700$ & 600 & $1.454 \mathrm{e}-13$ & 81.835 \\
\hline $700-800$ & 700 & $3.614 \mathrm{e}-14$ & 124.64 \\
\hline $800-900$ & 800 & $1.170 \mathrm{e}-14$ & 181.05 \\
\hline $900-1000$ & 900 & $5.245 \mathrm{e}-15$ & \\
\hline
\end{tabular}


Table A.2: Inclination dependent orbital debris function [44].

\begin{tabular}{l|l}
\hline Inclination (deg) & Psi \\
\hline $0-24$ & 1 \\
25 & 0.9000 \\
26 & 0.9050 \\
27 & 0.9100 \\
28 & 0.9120 \\
28.5 & 0.9135 \\
29 & 0.9150 \\
30 & 0.9200 \\
31 & 0.9220 \\
32 & 0.9270 \\
33 & 0.9300 \\
34 & 0.9350 \\
35 & 0.9400 \\
36 & 0.9450 \\
37 & 0.9500 \\
38 & 0.9520 \\
39 & 0.9570 \\
40 & 0.9600 \\
41 & 0.9670 \\
42 & 0.9720 \\
43 & 0.9770 \\
44 & 0.9820 \\
45 & 0.9900 \\
46 & 0.9950 \\
47 & 1.0000 \\
48 & 1.0050 \\
49 & 1.0100 \\
50 & 1.0200 \\
51 & 1.0250 \\
52 & 1.0300 \\
53 & 1.0400 \\
54 & 1.0450 \\
55 & 1.0500 \\
56 & 1.0600 \\
57 & 1.0650 \\
58 & 1.0750 \\
59 & 1.0800 \\
& \\
\hline &
\end{tabular}




\begin{tabular}{l|l} 
Inclination (deg) & Psi \\
\hline 60 & 1.0900 \\
61 & 1.1000 \\
62 & 1.1150 \\
63 & 1.1300 \\
64 & 1.1400 \\
65 & 1.1600 \\
66 & 1.1800 \\
67 & 1.2000 \\
68 & 1.2200 \\
69 & 1.2400 \\
70 & 1.2600 \\
71 & 1.2900 \\
72 & 1.3100 \\
73 & 1.3400 \\
74 & 1.3800 \\
75 & 1.4100 \\
76 & 1.5000 \\
77 & 1.6300 \\
78 & 1.6800 \\
79 & 1.7000 \\
80 & 1.7100 \\
81 & 1.7000 \\
82 & 1.6800 \\
83 & 1.6100 \\
85 & 1.5300 \\
86 & 1.4900 \\
87 & 1.4500 \\
88 & 1.4100 \\
89 & 1.3900 \\
90 & 1.3800 \\
91 & 1.3700 \\
92 & 1.3800 \\
93 & 1.4000 \\
94 & 1.4400 \\
95 & 1.5000 \\
& \\
\hline 5
\end{tabular}




\begin{tabular}{l|l} 
Inclination (deg) & Psi \\
\hline 96 & 1.6400 \\
97 & 1.7000 \\
98 & 1.7500 \\
99 & 1.7700 \\
100 & 1.7800 \\
101 & 1.7700 \\
102 & 1.7500 \\
103 & 1.7200 \\
104 & 1.6900 \\
105 & 1.6600 \\
106 & 1.6100 \\
107 & 1.5600 \\
108 & 1.5100 \\
109 & 1.4600 \\
110 & 1.4100 \\
111 & 1.3800 \\
112 & 1.3500 \\
113 & 1.3200 \\
114 & 1.3000 \\
115 & 1.2800 \\
116 & 1.2600 \\
117 & 1.2400 \\
119 & 1.2200 \\
120 & 1.2000 \\
121 & 1.1800 \\
122 & 1.1650 \\
123 & 1.1550 \\
125 & 1.1400 \\
$126-180$ & 1.1250 \\
& 1.1100 \\
19 &
\end{tabular}


B. DSMC Results

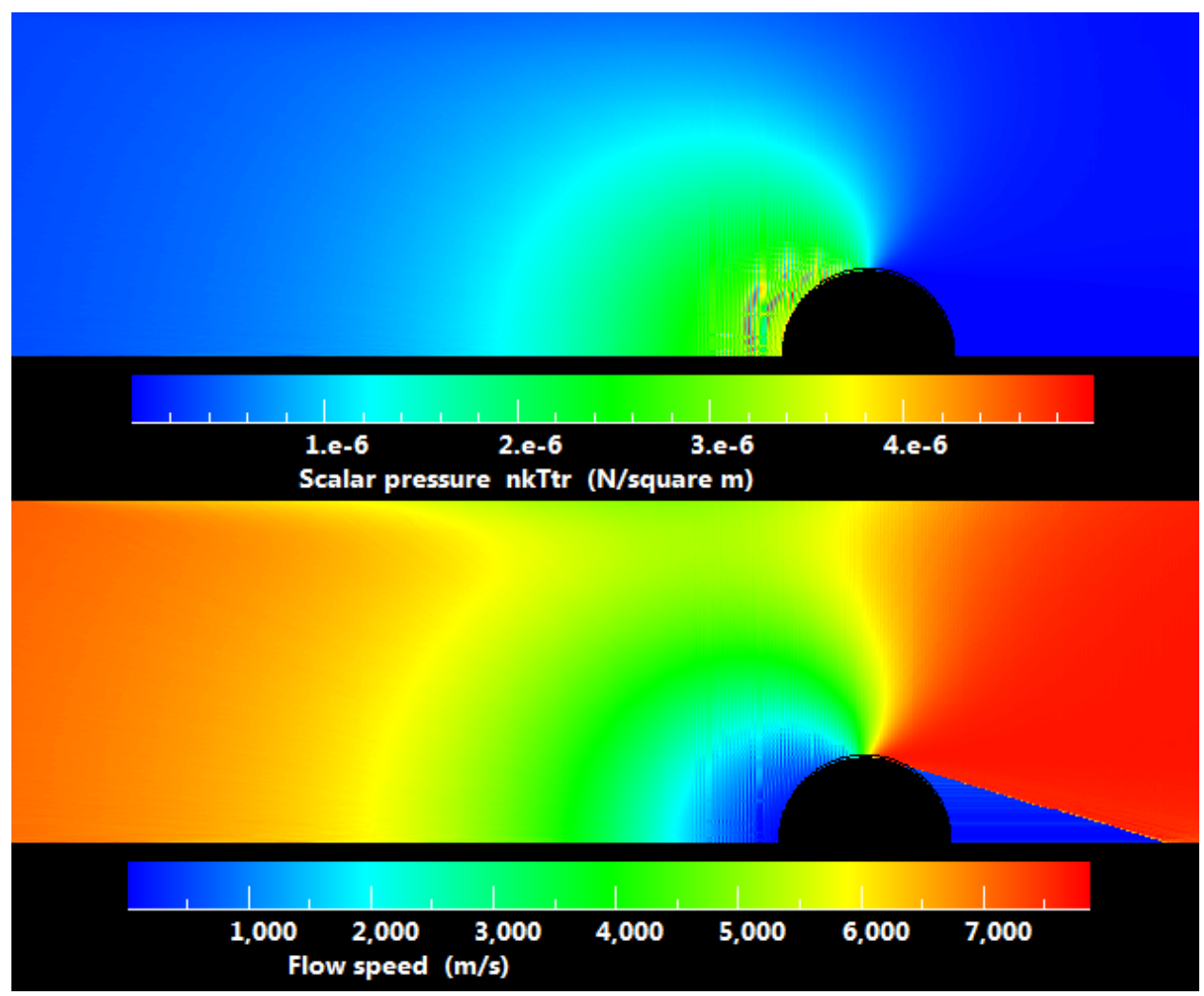

Figure B.1: Flow pressure (top) and velocity (bottom) results from a DSMC simulation at $500 \mathrm{~km}$ altitude. 
Table B.1: DSMC results for drag coefficient and dynamic pressure

\begin{tabular}{l|l|l}
\hline Altitude $(\mathrm{km})$ & Coefficient of Drag & Dynamic Pressure $(\mathrm{Pa})$ \\
\hline 100 & 1.458 & 31 \\
110 & 1.802 & 5.1 \\
120 & 1.915 & 1.2 \\
130 & 1.980 & 0.43 \\
140 & 2.023 & 0.21 \\
150 & 2.048 & 0.12 \\
160 & 2.064 & 0.07 \\
170 & 2.074 & 0.046 \\
180 & 2.082 & 0.032 \\
190 & 2.087 & 0.022 \\
200 & 2.091 & 0.016 \\
210 & 2.094 & 0.012 \\
220 & 2.098 & 0.0085 \\
230 & 2.098 & 0.0065 \\
240 & 2.101 & 0.005 \\
250 & 2.102 & 0.004 \\
275 & & 0.0022 \\
300 & & 0.0013 \\
325 & & 0.00075 \\
350 & & 0.00046 \\
375 & & 0.00028 \\
400 & & 0.00019 \\
425 & & 0.00013 \\
450 & & $8.5 \mathrm{e}-05$ \\
500 & & $3.8 \mathrm{e}-05$ \\
550 & & $1.8 \mathrm{e}-05$ \\
600 & & $9.5 \mathrm{e}-06$ \\
800 & & $2.8 \mathrm{e}-06$ \\
900 & & $9.7 \mathrm{e}-07$ \\
1000 & & $4.2 \mathrm{e}-07$ \\
& & $2.2 \mathrm{e}-07$ \\
\hline 00 & &
\end{tabular}

